\title{
VIRULENCE RESPONSE OF ESCHERICHIA COLI O157:H7 TO BILE SALTS AND SHORT CHAIN FATTY ACIDS
}

\author{
by \\ Mihaela Anca Serbanescu \\ Master of Science, University of Bucharest, 2001
}

\author{
A thesis \\ presented to Ryerson University \\ in partial fulfillment of the \\ requirements for the degree of \\ Master of Science \\ in the Program of \\ Molecular Science \\ Toronto, Ontario, Canada, 2008 \\ (C) Mihaela Anca Serbanescu 2008 \\ PROPERTY OF \\ RYERSON UNIVERSITY LIBRARY
}




\section{Author's Declaration}

I hereby declare that I am the sole author of this thesis.

I authorize Ryerson University to lend this thesis to other institutions or individuals for the purpose of scholarly research.

I further authorize Ryerson University to reproduce this thesis by photocopying or by other means, in total or in part, at the request of other institutions or individuals for the purpose of scholarly research. 


\title{
VIRULENCE RESPONSE OF ESCHERICHIA COLI O157:H7 TO BILE SALTS AND SHORT CHAIN FATTY ACIDS
}

\author{
Mihaela Anca Serbanescu \\ Master of Science, Molecular Science, Ryerson University \\ 2008
}

\section{Abstract}

Enterohemorrhagic Escherichia coli (EHEC) $0157: \mathrm{H} 7$ is a gastrointestinal pathogen which causes hemorrhagic colitis and can lead to neurological damage, acute kidney failure (hemolytic uremic syndrome) and vascular lesions. During intestinal colonization EHEC is exposed to a variety of stresses including bile salts (BS) in the small intestine and short chain fatty acids (SCFA) in the large intestine; little is known about how these stresses affect this pathogen's virulence properties. The goal of this study was to investigate the impact of exposure of E. coli $\mathrm{O} 157: \mathrm{H} 7$ to physiologically relevant concentrations of $\mathrm{BS}$ and SCFA alone and in mixtures on bacterial survival, verotoxin production and adhesion to human epithelial cells. The results indicated that BS treatments significantly enhanced several virulence properties including survival and adhesion to human epithelial cell lines including colonic epithelial cells. Verotoxin production was not affected by any of the BS treatments. Bacterial pretreatment with erythromycin at a sub-minimal inhibitory concentration eliminated the adhesion enhancement after BS treatment, suggesting that protein synthesis was required for enhanced adhesion of BS treated organisms. Using the isogenic mutant of the known adhesins, intimin and iha it was established that there was no role for intimin or iha in the BS-induced adhesion enhancement. SCFA treatments reduced bacterial viability but significantly enhanced both adhesion to human epithelial cells and verotoxin production. The results of this research indicate that ingestion stresses such as BS and SCFA, which are part of the host's natural chemical assault on foreign organisms, may actually enhance the virulence properties of this pathogen and contribute to, rather than, prevent infection. Furthermore, they suggest that this pathogen may use these ingestion stresses to cue the expression of numerous virulence factors for successful infection of local microenvironments. 


\section{Acknowledgements}

I would like to thank especially to my supervisor, Dr. Debora Foster who has truly represented a guide and stimulus towards the best that I could produce and for her encouragement and patience throughout the duration of this thesis project. I would also like to thank Dr. Nita Prayitno, who was the initial mentor and early on providing advice and encouragement and who worked and generously shared data with me. I also need to acknowledge my supervisory committee members, Dr. Gideon Wolfaardt and Dr. Kim Gilbride for their critiques and valuable advice pertaining to my research.

I am also thankful for the support, help and use of laboratory space and equipment throughout the course of this research of Beth Binnington, Lingwood laboratory of the Hospital for Sick Children and the Dr. Wolfaardt laboratory at Ryerson University. Furthermore, I would like to thank everyone in my lab, particularly fellow students working under the supervision of Dr. Foster, for their continued support and assistance during this project. I am of course very thankful of Dr. Julianne Kus's level of critique, assistance and willingness to discuss aspects of the study with me or to obtain insight in wider context.

I am grateful for scholarship funding from the Ontario Graduate Scholarship Program and the School of Graduate Studies at Ryerson University which made this research thesis financially possible.

Finally, it is not possible to give enough thanks to my husband Lucian, without whose unfailingly loving, financial and emotional support this work would certainly have faltered. Indeed, it may never have been undertaken at all. I am grateful to him.

Thank you everyone!!!! 


\section{Table of Contents}

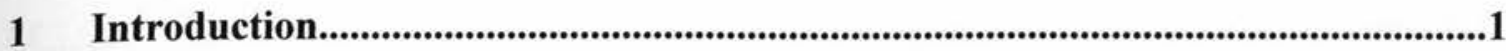

$1.1 \quad$ Enterohemorrhagic Escherichia coli ...................................................................

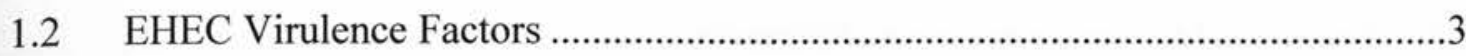

1.2.1 Shiga Toxins/Verotoxins ......................................................................

1.2.2 Adherence to Host Cells …….....................................................................

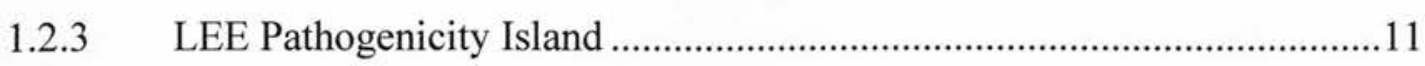

1.2.4 Type Three Secretion System ....................................................................12

1.2.5 TTSS Effectors ………………………….........................................13

1.2.6 Non LEE Encoded Effectors .....................................................................13

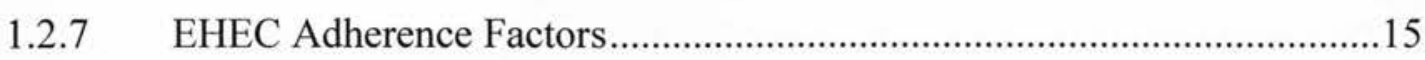

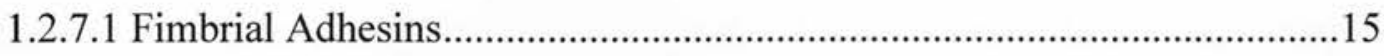

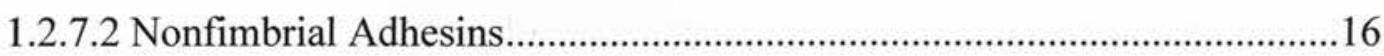

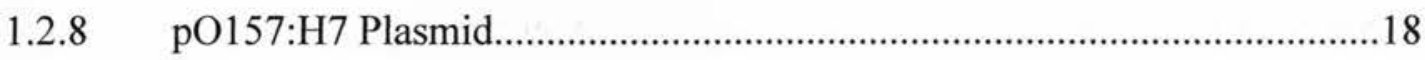

1.2.9 Regulation of the EHEC Virulence Genes .................................................19

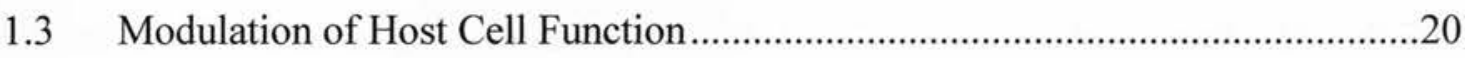

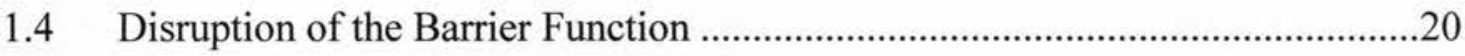

1.4.1 Factors Contributing to Diarrhea ................................................................21

1.4.2 Host Cell Cytoskeletal Rearrangements ....................................................21

1.5 Impact of Ingestion Stress on Virulence............................................................24

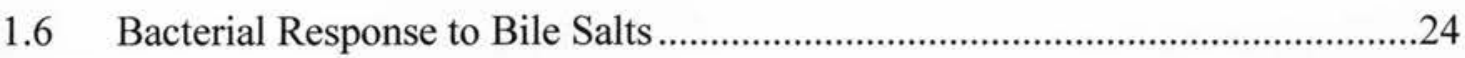

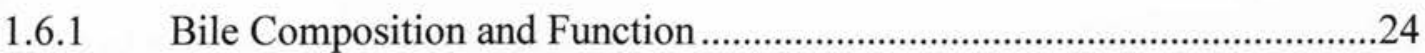

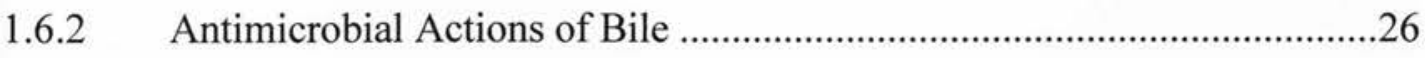

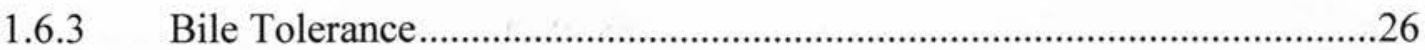

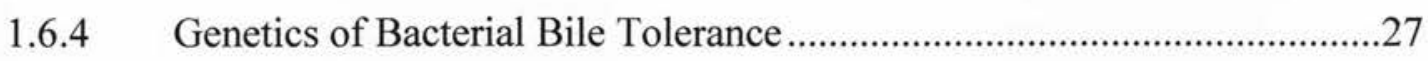

1.6.5 Bile Sensing and Regulation of Bile Stress Response.................................28

1.6.6 Bile and Pathogenesis ..................................................................................28

1.7 Short Chain Fatty Acid Stress Response ………..................................................30

1.7.1 Short Chain Fatty Acid Composition and Function ...................................30

1.7.2 Short Chain Fatty Acid Tolerance and Pathogenesis ...................................31 


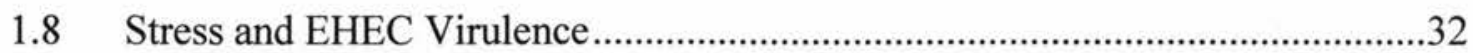

1.9 Purpose of Investigation and Hypothesis ..........................................................33

1.9.1 Research Rationale ...................................................................................3

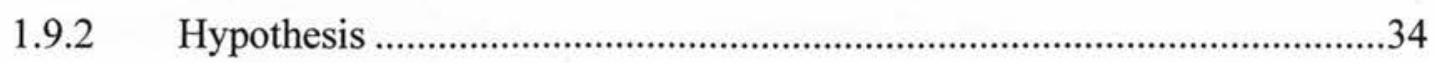

1.9.3 The Main Objectives of this Thesis ..............................................................34

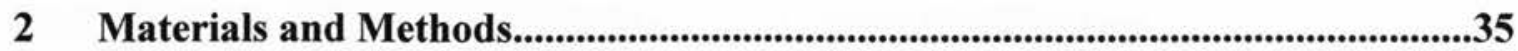

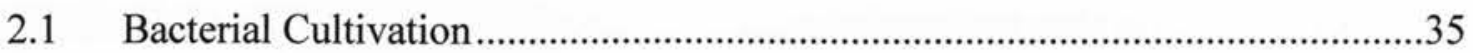

2.2 Tissue Cell Culturing ...................................................................................

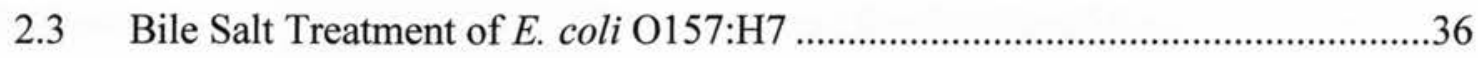

2.4 Short Chain Fatty Acid Stress of E. coli O157:H7 ............................................38

$2.5 \quad$ Percent Survival of Treated E. coli O157:H7 ……...........................................39

2.6 Bacteria- Host Cell Adhesion Assay: Colony Plate Count Method .....................39

2.7 Impact of Erythromycin-Mediated Inhibition of Protein Synthesis on Bacteria-

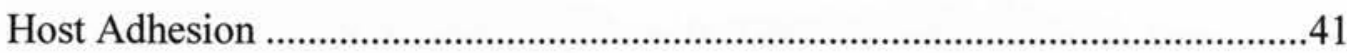

2.8 Bile Salt/Short Chain Fatty Acids Treated E. coli O157:H7 86-24 Growth during Infection Time ....................................................................................

2.9 Qualitative Microscopic HEp-2 cell Adhesion Assay after Exposure to Bile Salts/Short Chain Fatty Acids

2.10 Vero Cell Cytotoxicity Assays …………….....................................................

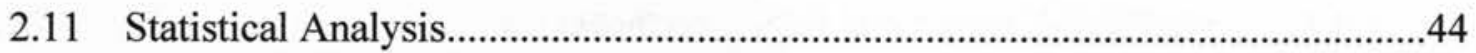

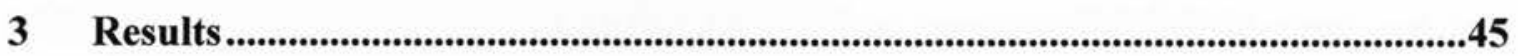

3.1 Bile Salt Treatment Enhances E. coli O157:H7 Survival.................................45

3.2 Bile Salt Treatment Enhances E.coli O157:H7 Adhesion to Human Epithelial Cells.

3.3 Erythromycin Treatment Abrogates EHEC 0157:H7 Adhesion Enhancement.48

3.4 No Role of Intimin in Enhanced Adhesion of BS Treated EHEC O157:H7 ......49

3.5 No Role of Tha in Enhanced Adhesion of Bile Salt Treated EHEC O157:H7 ...52

3.6 Short Chain Fatty Acids Decrease E. coli O157:H7 Viability ............................55

3.7 Short Chain Fatty Acids Stress Enhances E. coli O157:H7 Adhesion to Human Epithelial Cells. 
3.8 Erythromycin Treatment Reduces Short Chain Fatty Acids Host Adhesion

Enhancement.

3.9 The Effect of Bile Salts and Short Chain Fatty Acids Stress on E. coli O157:H7

86-24 Growth during Infection Time

3.10 No Change in Secreted and Periplasmic VT Production after BS Treatment ....61

3.11 VT Levels Increased after SCFA Mixture. 66

4 Discussion 70

4.1 Effect of Intestinal Stress on E. coli O157:H7 Survival: Summary ...................70

4.1.1 Bile Salt Mix Treatment Enhances E. coli O157:H7 Survival ...................70

4.1.2 Short Chain Fatty Acids Treatment Reduces E. coli O157:H7 Survival...72

4.2 BS Mix Treatment Enhances E. coli O157:H7 Adhesion to Epithelial Cells ....73

4.3 Short Chain Fatty Acid Stress Enhances Adhesion of E. coli O157:H7 to

Epithelial Cells.

4.4 The Effect of Bile Salts and Short Chain Fatty Acids Treatments on E. coli O157:H7 Growth during Infection Time

4.5 The Effect of Bile Salts and Short Chain Fatty Acids Treatment on E. coli O157:H7 86-24 Verotoxin Production:

4.5.1 No Change in Secreted and Periplasmic VT Production after Bile Salt

Treatment 80

4.5.2 Increased VT Levels after Short Chain Fatty Acids Mixture Stress ..........81

5 Summary and Conclusions ............................................................................................82

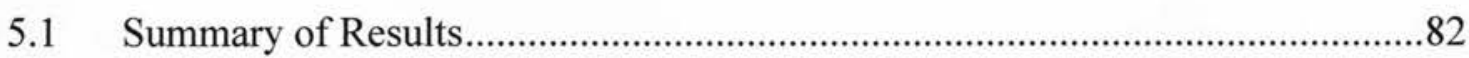

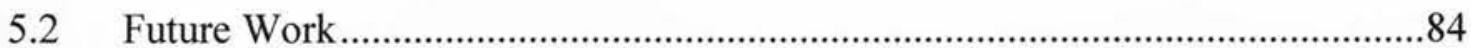

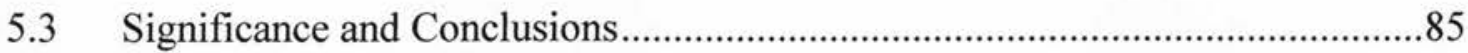




\section{List of Tables}

Table 1-1 Bile Salts in Duodenum 25

Table 2-1 Bacterial Strains Used in this Study 35

Table 2-2 Cell Lines Used in This Study 36

Table 2-3 Bile Salt Experimental Treatments 38

Table 2-4 Short Chain Fatty Acid Stress Experimental Treatments 39

Table 6-1 Supplemental Data $1 \quad 87$

Table 6-2 Supplemental Data $2 \quad 88$ 


\section{List of Figures}

Figure 1.2-1 EHEC genes involved in pathogenesis. 4

Figure 1.2-2 Ribbon crystal structure of E. coli O157:H7 Stx2. 6

Figure 1.2-3 Simplified cartoon of the mode of action of Stx. B. 8

$\begin{array}{ll}\text { Figure 1.2-4 Model for EHEC Adhesion to Host Cells. } & 10\end{array}$

Figure 1.2-5 Genetic organization of LEE genes in EHEC. 12

Figure 1.2-6 Simplified cartoon model for EHEC translocation of LEE encoded and $\begin{array}{ll}\text { other proteins into the host cell cytosol. } & 14\end{array}$

Figure 1.4-1 EPEC and EHEC translocate effector proteins into the host cell cytosol. 23

Figure 3.1-1 BS Treatment Enhances EHEC O157:H7 Survival 45

Figure 3.2-1 BS Treated E. coli O157:H7 86-24 Show Enhanced Adhesion to HEp-2 Cells

Figure 3.2-2 Qualitative Comparison of BS Treated E. coli O157:H7 86-24 Adhesion to HEp-2 Cells $\quad 47$

Figure 3.3-1 Erythromycin Abrogates E. coli O157:H7 86-24 Adhesion Enhancement to Caco-2 Cells.

Figure 3.4-1 BS Treated E. coli O157:H7 86-24 eae $\Delta 10$ Show Enhanced Adhesion to HEp-2 Cells

Figure 3.4-2 BS Treated E. coli O157:H7 8624 eae $\Delta(\mathrm{pEB} 310)$ Show Enhanced Adhesion to HEp-2 Cells.

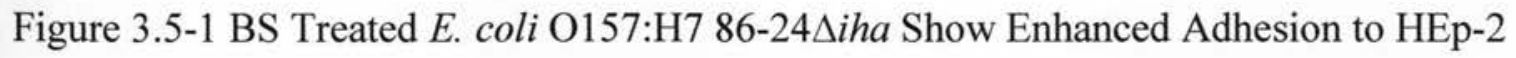
Cells

Figure 3.5-2 BS Treated E.coli ORN 172(pIHA) and (pSK+) Adhesion to HEp-2 Cells 55

Figure 3.6-1 SCFATreatment Decreases EHEC 0157:H7 86-24 Survival 56

Figure 3.7-1 SCFA Shocked E. coli O157:H7 86-24 Show Enhanced Adhesion to HEp-2 Cells

Figure 3.9-1 The Effect of BS Treatment on E. coli O157:H7 86-24 Viability during $4 \mathrm{~h}$ Incubation 
Figure 3.9-2 The Effect of SCFA Stress on E. coli O157:H7 86-24 Viability during $4 \mathrm{~h}$ Incubation

Figure 3.10-1 Secreted Verotoxin Production of E. coli O157:H7 86-24 after Exposure to BS

Figure 3.10-2 Periplasmic Verotoxin Production of E. coli O157:H7 86-24 after Exposure to BS

Figure 3.10-3 Initial and Final Bacteria Concentrations of BS Treated E. coli O157:H7 86-24 during Verotoxin Production

Figure 3.11-1 Secreted Verotoxin Production of SCFA Stressed E. coli O157:H7 86-2467

Figure 3.11-2 Secreted Verotoxin Production of SCFA Stressed E. coli O157:H7 86-2468

Figure 3.11-3 Initial and Final Bacteria Concentrations of SCFA Stressed E. coli O157:H7 86-24 during Verotoxin Production 


\section{Abbreviations}

$\mathrm{A} / \mathrm{E}$

AcrAB pump

ATCC

BS

CaCo-2

$\mathrm{CFU}$

CT

Da

dd $\mathrm{H} 2 \mathrm{O}$

DMEM

EDTA

Efa-1

EHEC

FBS

Gb-3

GI

$\mathrm{HC}$

HEp-2

HUS

HUVEC

Iha

LB

LEE

Ler

LPS

MEM

MLC

$\mathrm{NaCDC}$
Attaching and effacing lesions

Drug efflux pumps

American Type Culture Collection

Bile salts

Colonic adenocarcinoma cells

Colony forming units

Cholera toxin

Daltons

Distilled deionized $\mathrm{H} 2 \mathrm{O}$

Dulbecco's MEM

Ethylenediaminetetraacetic acid

EHEC factor for adherence

Enterohemorrhagic Escherichia coli

Fetal bovine serum

Glycolipid globotriaosylceramide-3 receptor

Gastrointestinal tract

Hemorrhagic colitis

Laryngeal carcinoma cells

Hemolytic-uremic syndrome

Human umbilical vein endothelial cells

IrgA homologue adhesion

Luria-Bertani

Locus of enterocytes effacement LEE

LEE-encoded regulator

Lipopolysaccharide

Minimal essential media

Myosin light chain

Chenodeoxycholate 
$\mathrm{NaDOC}$

Deoxycholate

$\mathrm{NaGIC}$

Glycholate

NaUDC

Ursodeoxycholate

$\mathrm{N}$-WASP

Neuronal Wiskott-Aldrich syndrome protein

OmpA

Outer membrane protein $\mathrm{A}$

$\mathrm{OmpF}$

OmpC Porin proteins

PAI

Large pathogenicity island

PBS

Phosphate buffered saline

perC

Plasmid encoded regulator

PKC

Protein kinase C

RpoS

Stress response regulon

Saa

Autoagglutinating adhesion

SCFA

Short chain fatty acids

SFP

Sorbitol fermenting EHEC 0157 fimbriae plasmid

STEC

Shiga toxin producing E. coli

Stx

Shiga toxin

TccP

Tir-cytoskeleton coupling protein

TCP

Production and toxin-coregulated pilus

TCSs

Two-component system

Tir

Transmembrane intimin receptor

Tir

Translocated intimin receptor

ToxT

Transcription factor

TTSS

Type III secretion system

Vero cells

African Green Monkey Kidney cells

VT

Verotoxin

wt

Wild type

$\sigma \mathrm{B}$

Sigma B transcriptional regulator

$\sigma \mathrm{S}$

RpoS trascriptional regulator 


\section{Introduction}

\subsection{Enterohemorrhagic Escherichia coli}

Enterohemorrhagic Escherichia coli (EHEC) are food-borne pathogens which primarily colonize the human intestinal tract. They are Gram-negative bacteria that belong to the Shiga toxin producing E. coli (STEC) family. EHEC can cause a wide range of illnesses ranging from mild diarrhea to hemorrhagic colitis (HC) which can progress to several life threatening diseases including hemolytic-uremic syndrome (HUS) and thrombotic thrombocytopenic purpoura (Wells et al., 2008). E. coli strain O157:H7, designated by the somatic antigen $\mathrm{O}$ and the flagellar antigen $\mathrm{H}$, was first recognized as a pathogen in 1982. It is the most well studied serotype of the group since it is most commonly associated with severe EHEC infections and also has been linked to outbreaks of illness worldwide. EHEC infections lead to a high proportion of deaths due to HUS (with mortality rate of this complication of 3-17\%), and cause a great deal of morbidity with many surviving individuals experiencing chronic renal insufficiency and neurological damage (Nataro \& Kaper, 1998).

The production of Shiga toxin (Stx) and the presence of a pathogenicity island that encodes the locus of enterocytes effacement (LEE) are virulence factors that enable $E$. coli $\mathrm{O} 157: \mathrm{H} 7$, to cause the severe gastrointestinal infections with the potential lifethreatening complications (Wells et al., 2008). Nonpathogenic E. coli strains do not possess these genetic characteristics that may contribute to specific virulence traits or to successful survival and fitness of the bacteria in the host (Hacker \& Kaper, 2000). Cattle have been identified as the most important reservoir of EHEC/STEC (Naylor et al., 2003; Low et al., 2006). Common routes of transmission include fecal transmission and contaminated food or water (Caprioli et al., 2005; Ceponis et al., 2005). Although most outbreaks are associated with undercooked ground beef, recently many of sources of infection have originated from vegetables including lettuce, spinach, cabbage, sprouts or tomatoes (Borczyk et al., 1987). Other outbreaks have also been reported after the consumption of contaminated fruits, water or among persons visiting farms, petting zoos 
and via direct contact in hospital and daycare settings (Louie et al., 2000). EHEC is a highly efficient pathogen in humans since the infective dose is estimated to be only approximately 50-100 organisms (Griffin and Miner, 1995). The ability of EHEC to persistently colonize the gastrointestinal tract (GI) relies on its capability to sense and respond to many stressful environments (Maroncle et al., 2006). E. coli 0157:H7 attachment to the epithelial cells is the first critical step in colonization and is mediated by bacterial adhesins. In order to colonize the human colon, EHEC initially adheres nonintimately, followed by intimate attachment to the host cell (Torres et al., 2005b).

It has been proposed that initial attachment of EHEC to the epithelial cells is mediated by EspA filaments as well as fimbrial adhesions, including LP fimbriae (Kenny, 1999). The Esp effector proteins and transmembrane intimin receptor (Tir) are translocated via the multicomponent apparatus type III secretion system (TTSS) into the human host. This initial adherence of the bacteria to the target cells is followed by the intimate attachment which is associated with the binding of the bacterial adhesin intimin to the Tir (Kenny, 1999).

The ability of E. coli $\mathrm{O} 157: \mathrm{H} 7$ to promote the development of attaching and effacing (A/E) lesions has been attributed to the presence of a pathogenicity island on the bacterial genome. The LEE pathogenicity island encodes the genes products responsible for the generation of $\mathrm{A} / \mathrm{E}$ lesions on intestinal epithelial cells (Elhanafi et al., 2004). Apart from the LEE encoded proteins, several adhesins including adherence conferring molecule (Iha) (Tarr et al., 2000), long polar fimbriae (lpf) (Torres et al., 2002), curli (Uhlich et al., 2001) and F9 fimbriae (Dziva et al., 2004; Low et al., 2006) are associated with adherence to mammalian cells. The production of Stx (verocytotoxin) is one the major virulence characteristics of EHEC since the toxins are associated with development of HC and HUS due to EHEC infections (Law, 2000; Caprioli et al., 2005). HUS arises as a consequence of microvascular disease triggered by the toxins entering the bloodstream and binding to the receptor Gb3 of kidney and brain cells (Spear et al., 2006).

In spite of significant advances made in understanding EHEC pathogenesis, we still do not fully appreciate the impact of environmental stresses on virulence factor production and the influence this may exert on pathogenesis. Following consumption, the 
acidity of the stomach, is a major barrier encountered by bacteria. The EHEC that survive the exterme low $\mathrm{pH}$ then transit on to the intestines, where they must overcome a different set of biological barriers including bile salts in the small intestine and short chain fatty acids in the large intestine. As a consequence of these extreme environmetal changes, the bacteria must adapt physiologically and morphologically in order to survive. Questions on whether these changes influence major virulence properties, including host cell adhesion and verotoxin production, remain to be answered. To properly address these questions a multidisiplinary approach that includes molecular, cellular and microscopic techniques to identify pathogen-induced changes in infected cells will be needed. The research presented in this thesis will focus on the impact of bile salt and short chain fatty acid stress on the virulence of EHEC. The literature review will provide details of the pathogenic mechanism as well as known and postulated virulence factors, followed by a review of the effect of BS and SCFA stress on virulence of other related gastrointestinal pathogens.

\subsection{EHEC Virulence Factors}

The ability of $E$. coli $\mathrm{O} 157: \mathrm{H} 7$ to cause a spectrum of human illness, including $\mathrm{HC}$ and HUS has been attributed to the production of Shiga toxins (Stx) and the presence of a large pathogenicity island (PAI) (Wells et al., 2008). Genes involved in EHEC pathogenesis are presented in Figure 1.2-1. The pathogenicity island, termed the locus for enterocyte effacement (LEE), is a large multi-gene segment which encodes intimin (bacterial adhesin), its receptor Tir (translocated intimin receptor), a type III secretion machine and secreted proteins (EspA, EspB, EspD) necessary for the formation of attaching-and-effacing (A/E) lesions on intestinal epithelial cells (Elhanafi et al., 2004). The two primary classes of Stx (Stx1 and Stx2) are encoded by two type $\lambda$ lysogenic phages inserted in the bacterial genome (Elhanafi et al., 2004). In addition to Stx, the pO157 plasmid is known to encode the enterohemolysin (ehx) as well as a catalase peroxidase (katP), a zinc metallo protease (StcE), a serine protease (EsP) and a large toxin (ToxB) that contribute to EHEC pathogenesis (Herold et al., 2004). 


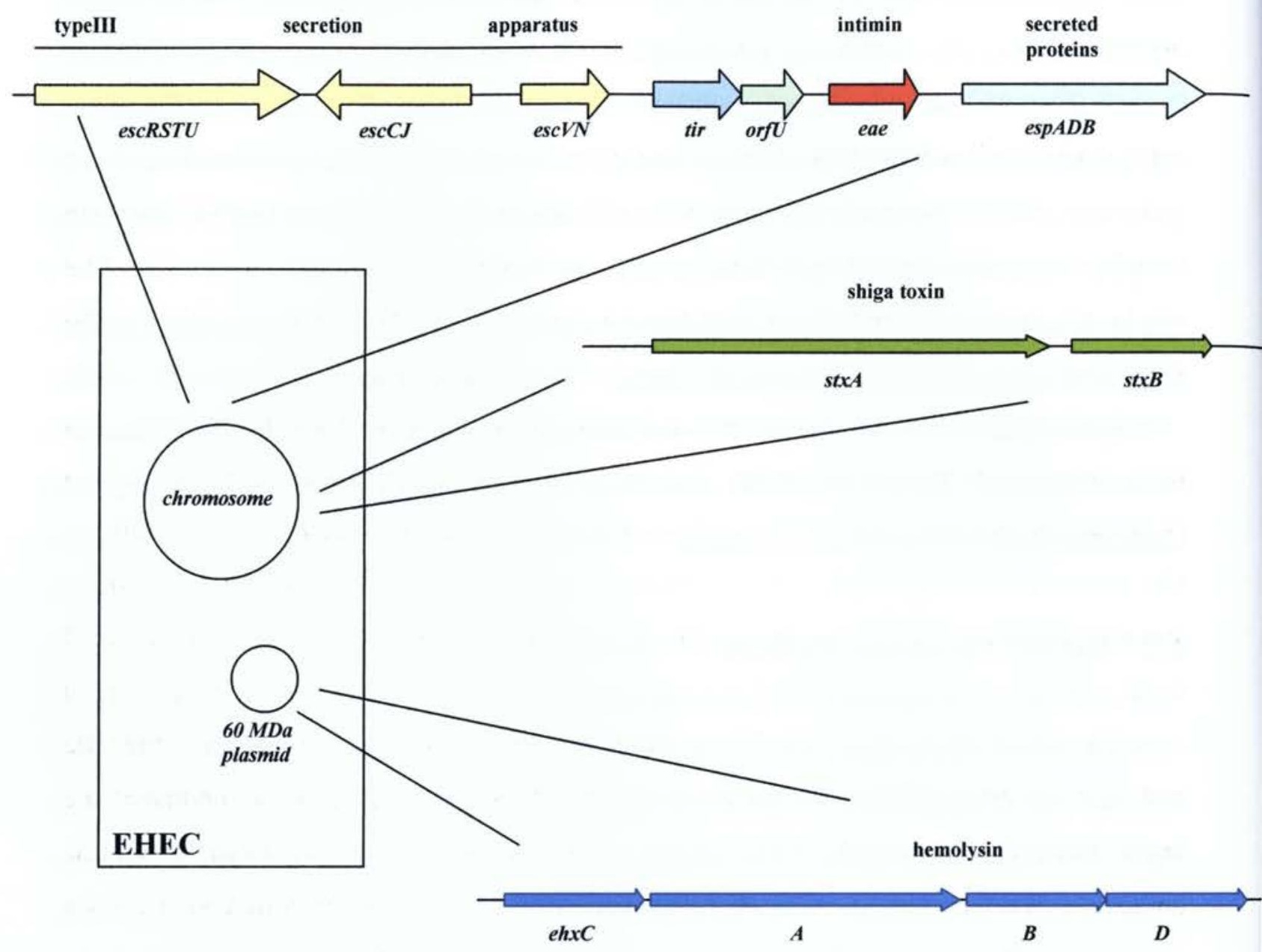

Figure 1.2-1 EHEC genes involved in pathogenesis.

Genes involved in EHEC pathogenesis are similar to those implicated in EPEC, except for the presence of the Stx-encoding phage on the EHEC chromosome and the presence of a EHEC plasmid which is known to encode enterohemolysin and other proteins involved in EHEC pathogenesis. Figure adapted from Nataro \& Kaper, 1998. 


\subsubsection{Shiga Toxins/Verotoxins}

One of the major pathogenicity factors of EHEC is the production of Shiga toxins (Stx), which are responsible for the major symptoms of $\mathrm{HC}$ and a sequela called hemolytic uremic syndrome (HUS) (Kaper et al., 1998). Stxs act to inhibit the synthesis of host cell proteins and contribute to vascular complications of HC. HUS arises as a consequence of microvascular disease triggered by the toxins entering the bloodstream by clathrin-dependent endocytosis and binding to a cell surface receptor of kidney and brain cells (Hoey et al., 2003). HUS is characterized by thrombocytopenia, hemolytic anemia and kidney lesions which can lead to a chronic renal failure and even death (Spear et al., 2006).

EHEC strains may produce two major toxins Stx 1 and Stx2 which are related to Stx from Shigella dysenteriae serotype 1 (O'Loughlin \& Robins-Browne, 2001; Garmendia et al., 2005), however, Stx2 is more frequently associated with HUS. The Stx1 form is highly conserved and homologous to Stx with only one amino acid difference in the A subunit. The Stx1 and Stx 2 A and B subunits are 68 and $73 \%$ similar at the amino acid level, respectively, and the crystal structures of Stx and Stx2 are remarkably similar. In contrast, Stx2 has numerous variants including Stx2c, Stx2d, Stx2e, and Stx2f which are distinguished by the receptor to which they bind, thus contributing to the ability of the toxin to target different cells (Fraser et al., 2004). The Stx/Stx1 and Stx2 differ from one another by the fact they are serologically distinct. The polyclonal antiserum to one toxin group shows no cross neutralization of the cytotoxicity of the other toxin (Strockbine $e t$ al., 1986; Wen et al., 2006).

Other studies have also shown that more severe infections are often associated with the expression of additional factors encoded on the LEE island and the plasmid pO157 (Nataro \& Kaper, 1998). Stx toxins are encoded by stxA and st $x \mathrm{~B}$ genes which are found on the lysogenic bacteriophages and expressed at neutral $\mathrm{pH}$ conditions similar to those of the intestinal tract as VT could be detected in faeces in natural and experimental infections (Karmali et al., 1983; Ramotar et al., 1995; Rahn et al., 1996), cattle (Montenegro et al., 1990; Ball et al., 1994; Hyatt et al ., 2001), pigs (Cornick et al., 2000), and mice (Isogai et al., 2000; Zhang et al., 2000). 
The expression of the stx genes is thought to be under the control of the E. coli SOS response which is induced by elevated level of intracellular single stranded DNA (Fraser et al., 2004).

Stx is an holotoxin (approximately 70 kilodaltons) formed by five identical B subunits that surround a single A subunit. A model of Stx2 is presented in Figure 1.2-2. Once produced in the lower intestine, Stx translocates across the intestinal epithelium, reaches the blood and targets cellular receptors (Paton \& Paton, 1998).

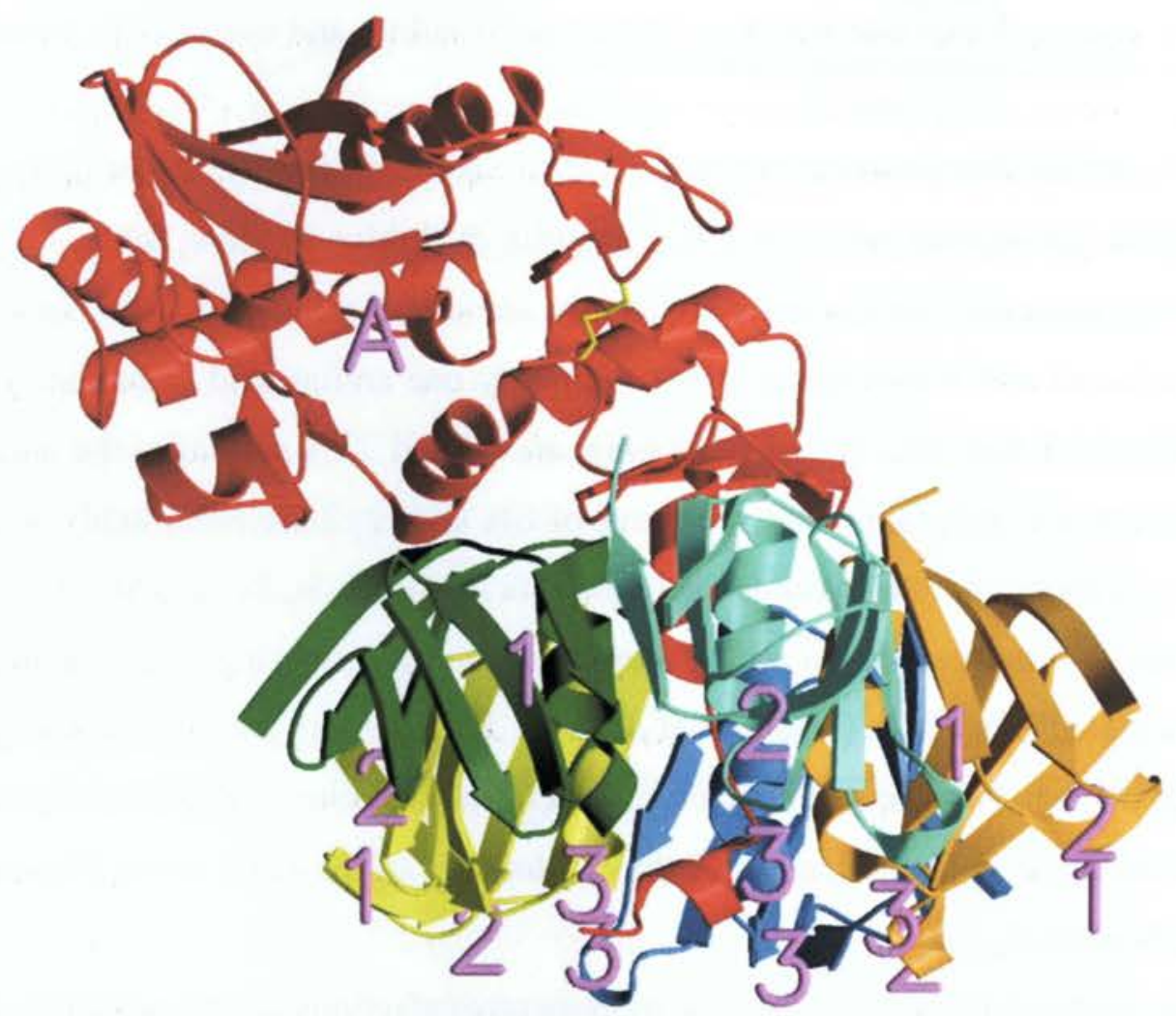

Figure 1.2-2 Ribbon crystal structure of E. coli O157:H7 Stx2.

The A-subunit is red, whereas the B-subunits are orange (chain B), cyan (chain C), green (chain D), yellow (chain E), and blue (chain F). The active site in the A-subunit is marked by the magenta letter A. The side chains of the cysteine residues that link A1 and A2 are depicted in yellow. The sites equivalent to the Gb3binding sites on the B-pentamer of Stx 1 are shown by magenta numbers that distinguish the type of binding site. Reproduced from Fraser et al., 2004. 
A simplified model which highlights the mode of action of Stx is presented in Figure 1.2-3. The B subunit $(7.7 \mathrm{kDa})$ is implicated in the binding to the glycolipid globotriaosylceramide-3 (Gb-3) receptor of endothelial cells referred as CD77. Following internalization, the toxin is transported to the trans Golgi network (Schmitt et al., 1999). Retrograde transport of toxin from the Golgi to the endoplasmic reticulum may also be required for the action of toxin to the cells (Sandvig et al., 1992; Sandvig et al., 1994). The A subunit of the toxins ( $32 \mathrm{kDa}$ ) is implicated in the inhibition of host protein synthesis and also activation and secretion of chemokines, leading to activation of cells of the immune system (Hoey et al., 2003). As result, the integrity of the epithelial barrier is altered and the translocation of Stx across the mucosa to underlying tissues is facilitated (Gobert et al., 2007). Shiga toxins also induce a cascade of responses including elevated levels of pro-apoptotic proteins, mitochondrial dysfunction and activation of apoptosis caspase cascades that lead to apoptosis in endothelial cells (Hoey et al., 2003). After translocation through the gut mucosa, Stxs first enter the bloodstream to reach the endothelial cells particularly those in the gut, kidney and occasionally other organs (Caprioli et al., 2005). Once the endothelial cells of the target organs are compromised, the toxins act on glomerular and brain microvascular endothelial cells and activate prothrombic and pro-inflammatory cascades, which lead to HUS and complications of the central nervous system (Caprioli et al., 2005). Shiga toxins are cytotoxic to human renal endothelial cells. The characteristic renal histopathology associated with Stx includes swollen glomerular endothelial cells and platelet and fibrin deposition within the glomeruli (Caprioli et al., 2005). It is a decrease in the rate of glomerular filtration that causes the acute renal failure associated with HUS (Paton \& Paton, 1998). One report in the literature showed that Stxs contribute to increased adherence to epithelial cells by increased surface expression of nucleolin and $\beta 1$ integrin which interact with intimin (Robinson et al., 2006). Their findings indicate that these eucaryotic proteins are present on the luminal surface of intestinal epithelia and are potentially accessible as receptors for intimin during EHEC 0157:H7 infection (Robinson et al., 2006). These data are consistent with another study by Barnett Foster et al. (2000) which reported that Stx upregulates the level of phosphatidyletanolamine (PE) outer membrane on the host cell which they identified as a candidate host receptor previously reported to bind EHEC. 
Recently, another study reported that Stx subverts the inflammatory response of human enterocytes by decreasing NF-kB (Gobert et al., 2007). These results highlight the possibility that Stx is an unrecognized inhibitor of the epithelial cell innate immune response (Gobert et al., 2007). Together these findings underline an essential role for Stx in the initial step of colonization of the intestinal mucosa. Some studies support the notion that most probably, the production of toxin alone is most likely insufficient to cause disease. Stx (both Stx1 and Stx2) when added to colonic epithelial cells, were shown to move across the cell layer without any cytotoxic effects, however when STEC strains were incubated with the cell monolayer, toxin(s) were transported through the cells at the same time and the cell monolayer was destroyed (Law, 2000). These observations suggest that STEC strains may produce other potent toxic products and/or there are possibly other bacterial factors that enhance Shiga toxin activity (Law, 2000).

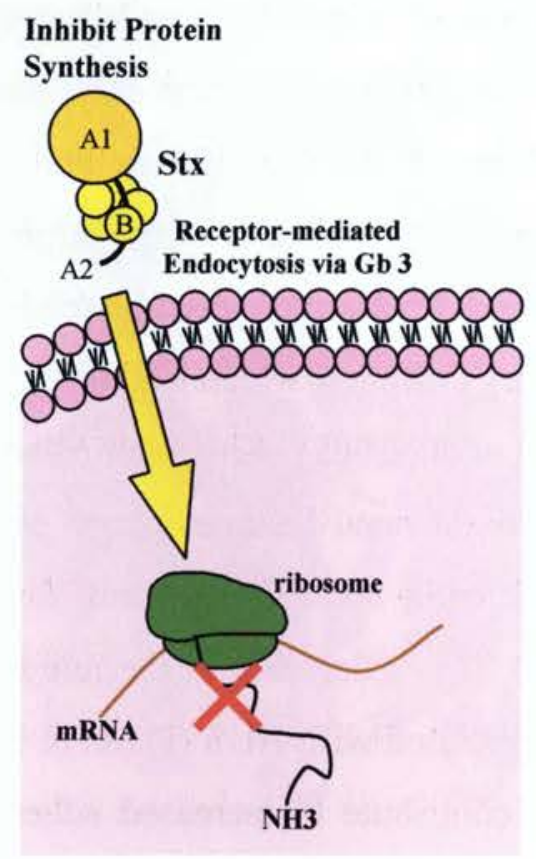

Figure 1.2-3 Simplified cartoon of the mode of action of Stx. B.

Inhibition of protein synthesis by Shiga toxins (Stx). Holotoxin, which consists of an enzymatically active (A) subunit and five binding (B) subunits, enters cells through the globotriasylceramide (Gb3) receptor. The $\mathrm{N}$-glycosidase activity of the A subunit then cleaves an adenosine residue from $28 \mathrm{~S}$ ribosomal RNA, which halts protein synthesis. Figure adapted from Schmitt et al., 1999. 


\subsubsection{Adherence to Host Cells}

For successful establishment of infection, E. coli $\mathrm{O} 157: \mathrm{H} 7$ use some remarkable strategies to adhere to host epithelial cells, survive peristaltic clearance and obtain access to derived nutrients of the distal ileum, cecum and colon. E. coli $\mathrm{O} 157: \mathrm{H} 7$ adhesion to the epithelial cells is an essential step in the diarrheal infection. EHEC adheres initially non-intimately to the intestinal epithelium surface and then intimately to enhance its attachment (Torres et al., 2005a). It has been proposed that initial attachment of EHEC to the epithelial cells is mediated by EspA filaments and fimbrial adhesions including long polar fimbria (Lpf) (Kenny, 1999). Following initial adherence, EHEC translocates various Esp effector proteins and Tir into the host via TTSS (Kenny, 1999). The initial adherence of the bacteria to the host cells is followed by the intimate attachment which is associated with the binding of the bacterial non-fimbrial adhesin (intimin) to its own injected receptor Tir (Kenny, 1999). After export to the surface, intimin is then integrated into the bacterial outer membrane with the amino-terminal region and the carboxyterminal region of the polypeptide exposed to the surface (Adams et al., 2005). From the bacterial surface, intimin projects a cell binding region which is positioned to contact the Tir (Adams et al., 2005). Apart from its interaction with Tir, intimin also recognizes and binds to host cell receptors such as integrin and nucleolin (Donnenberg et al., 1993; McKee et al., 1995; Robinson et al., 2006).

By adhering intimately to intestinal epithelial cells, EHEC has the capacity to induce a histopathological feature known as $\mathrm{A} / \mathrm{E}$ lesions. A/E lesions are distinguished by loss of intestinal microvilli, rearrangement of the cytoskeleton and formation of pedestal like structures that cup the bacterium (Campellone \& Leong, 2003; Caprioli et al., 2005). The proteins required for the formation of $\mathrm{A} / \mathrm{E}$ lesions are encoded by genes located on the chromosomally-encoded LEE pathogenicity island (Hayashi et al., 2001; Perna et al., 2001; Torres et al., 2005a). The LEE of EHEC consists of genes responsible for the expression of TTSS, intimin, Tir and secreted proteins such as EspA, EspB, EspD, EspG and Map (Torres \& Kaper, 2001; Spear et al., 2006). The TTSS is required for the translocation of various proteins (Esp) including Tir into the host cells. Once Tir has been translocated into the host cell, Tir moves to the plasma membrane where it forms a 
hairpin loop structure (Garmendia et al., 2005). While the amino- and carboxy-terminal domains are located within the cell, its central region is located at the surface of the host cell and allows binding of intimin (Garmendia et al., 2005). The cytosolic domains of Tir interact with cytoskeleton proteins which lead to formation of actin pedestal structures (Garmendia et al., 2005). A model for the early and later events associated with EHEC adherence to host cells is presented in Figure 1.2-4.

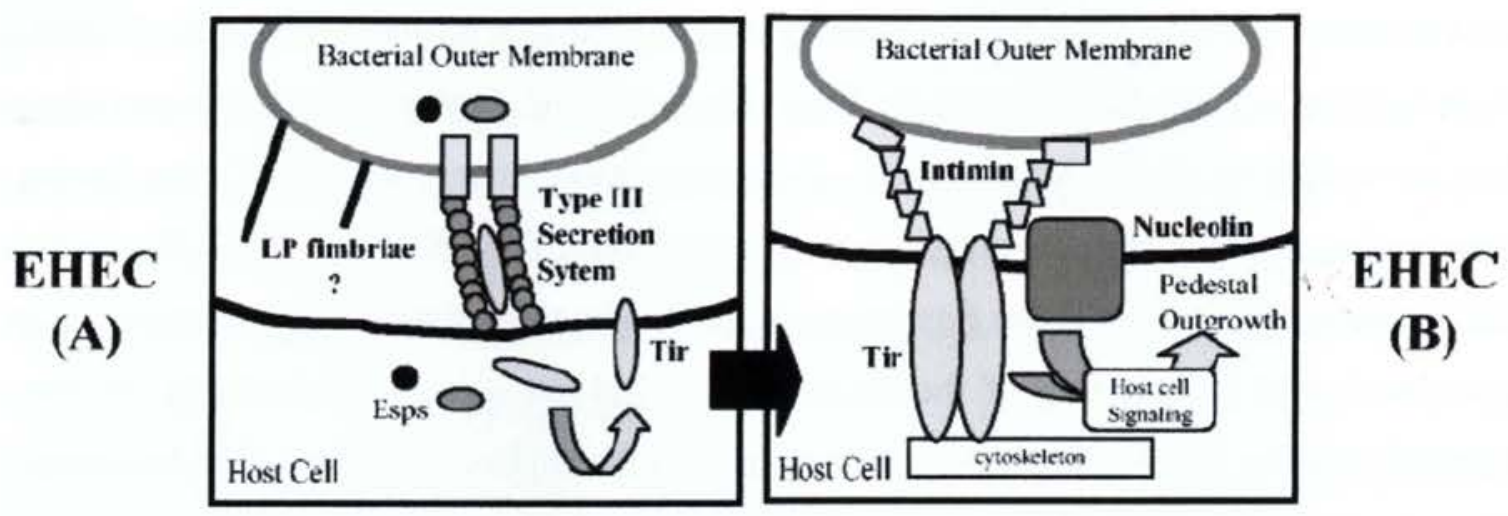

Figure 1.2-4 Model for EHEC Adhesion to Host Cells.

A: Early events in EHEC adhesion to host cells. EspA filaments of the TTSS and other possible adhesions such as LP fimbriae, mediate initial attachment of the bacteria to host cells. Various effector proteins (Esps), including Tir, are translocated into the host cell via the TTSS. Tir localizes to host plasma membrane and takes on a hairpin loop structure. B: Later events in EHEC adhesion to host cells. Intimate adhesion of the bacteria to the host cell is mediated by the binding of the bacterial adhesin intimin to its receptor Tir. Intimin also binds to a host cell receptor, nucleolin. Translocated effector proteins, including Tir, trigger cytoskeleton rearrangements, disruption of the epithelial barrier, cytotoxicity and are responsible for attaching and effacing (A/E) lesion formation, activating and inhibiting signal transduction pathways in host cells which can ultimately lead to disease. Reproduced from Torres et al., 2005b with the permission of Infection and Immunity, American Society for Microbiology. 


\subsubsection{LEE Pathogenicity Island}

Most of the genetic elements responsible for the formation of $\mathrm{A} / \mathrm{E}$ lesions are encoded within a $43 \mathrm{~kb}$ pathogenicity island in the 0157:H7 chromosome, named the locus of enterocyte effacement (LEE) (Nataro \& Kaper, 1998; Caprioli et al., 2005; Garmendia et al., 2005). EHEC are a subgroup of the Shiga-toxigenic E. coli (STEC) pathogroup which includes strains that produce Stx but may contain the LEE region or not. LEE negative EHEC strains have been associated with some cases of HUS, indicating that the LEE region is not essential for pathogenicity and that additional factors are involved in causing disease (Paton et al., 2001). The LEE region includes 5 major operons: LEE1, LEE2, LEE3, tir (LEE5), and LEE4 (Garmendia et al., 2005). A model of the LEE region that highlights the organization of the genes is presented in Figure 1.2-5. The 41 genes of LEE encode structural components of the TTSS, intimin, Tir, secreted proteins (EspA, B and D) and other TTSS structural proteins, translocators and effectors. The LEE operons are directly activated by the LEE-encoded regulator (Ler), which is the first gene in the LEE1 operon (Kaper et al., 2004). Regulation of LEE gene expression is complex and is under coordinated activities of three regulatory elements: Ler, GrlR and GrlA (Bustamante et al., 2001). Ler is a central activator necessary for the transcription of most of the LEE genes that is further positively and negatively modulated by GrlA and GrlR. The negative effect of GrlR on LEE expression depends on the GrlA function (Goldberg et al., 2001). Since recent studies have shown that LEE expression is under the control of various regulatory elements outside the locus, its regulatory mechanisms are complicated and require more research for clarification (Grant et al., 2003; Kaper et al., 2004). 


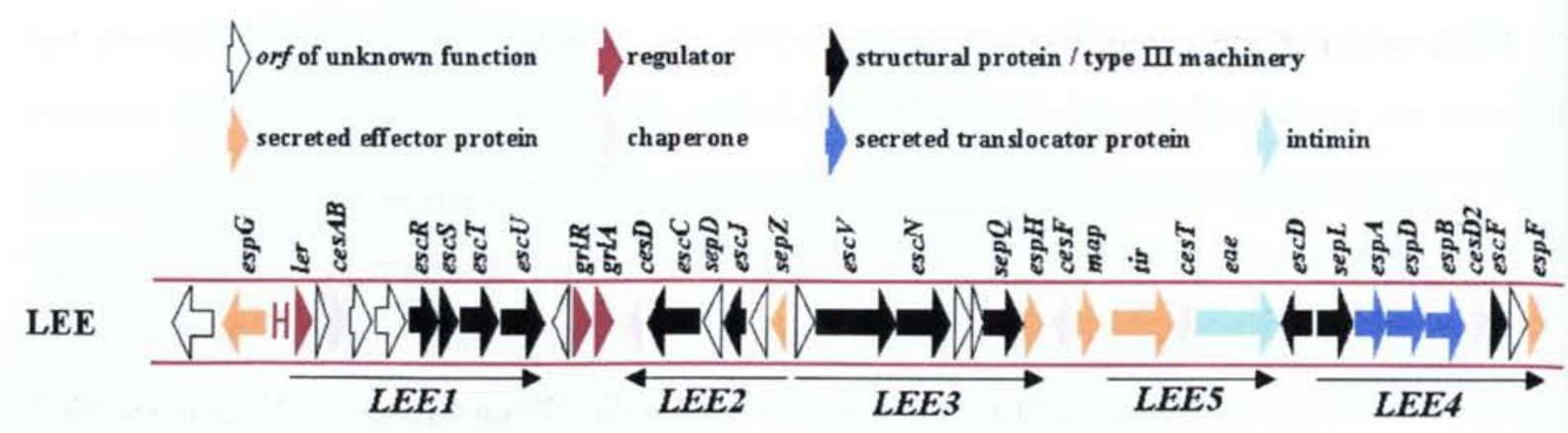

Figure 1.2-5 Genetic organization of LEE genes in EHEC.

The image was generated using coliBase (http://colibase.bham.ac.uk/). Reproduced from Garmendia et al., 2005 with the permission of Infection and Immunity, American Society for Microbiology.

\subsubsection{Type Three Secretion System}

In order to sabotage the cellular functions and establish the disease, E. coli $\mathrm{O} 157: \mathrm{H} 7$ uses highly sophisticated macromolecular syringes on its surface called type III secretion systems (TTSS). The TTSS apparatus, remarkable similar to a flagellar hook basal body complex, is composed of three distinct parts: a hollow extracellular needle, a cylindrical base body (which crosses two membranes and stabilizes the whole structure upon the cell envelope and bulb) and bulb (like the flagellar cytosolic complex composed of an ATPase and other proteins (Garmendia et al., 2005). EHEC uses this structure to penetrate and inject its toxins into host cells (Tampakaki et al., 2004; Pizarro-Cerdá \& Cossart, 2006). The basal body is composed of the secretin EscC and EscR, EscS, EscT, $\mathrm{EscU}$ and $\mathrm{EscV}$ which form the inner membrane proteins (Gauthier et al., 2003). The lipoprotein EscJ bridges the inner and outer membrane ring structures and EscF forms the needle complex of the TTSS (Wilson et al., 2001). EspA, EspB and EspD together constitute a needle like structure; EspA subunits polymerize to the tip to form the EspA filament and EspB and EspD make up the translocon pore (Delahay et al., 1999). SepD and SepL are cytoplasmic components of this system, and it has been suggested that these proteins may play a role in "switching" the type of proteins secreted through the TTSS, from translocator proteins to effector proteins (O'Connell et al., 2004). Cytoplasmic 
ATPases from the bacterial $\mathrm{F}_{0} \mathrm{~F}_{1}$ ATPase provides energy to the system and promotes protein secretion (Garmendia et al., 2005).

\subsubsection{TTSS Effectors}

Once the molecular syringe has been formed, the effector proteins are translocated into the host cell cytosol where they modulate the host cell signaling through molecular mimicry and hijack the host cell signaling events (Garmendia et al., 2005). A model of translocation of LEE encoded and other proteins into the host cell cytosol is presented in Figure 1.2-6. In addition, phosphorylation/dephosphorylation of critical residues in effectors often triggers the host cell signaling cascade (Garmendia et al., 2005). These result in cytoskeleton rearrangements (Tir, EspH, EspF and EspG), disruption of the epithelial barrier and tight junctions (EspF and Map), altered ion secretion, cytotoxicity (EspF and Cif), neutrophil recruitment, actin polymerization, disruption of the cell cycle, and transcriptional changes that will ultimately cause watery diarrhea (Dahan et al., 2004; Garmendia et al., 2005). Following the delivery of the effector proteins, EspA filaments and the needle-like complex are removed from the bacterial surface to allow intimate attachment of the bacteria and host. Once the bacteria are intimately attached, the LEEencoded genes are down-regulated (Garmendia et al., 2005).

\subsubsection{Non LEE Encoded Effectors}

In addition to the above described effector proteins, new effector proteins encoded on prophages or other PAIs have been found to be translocated into the host cell. TccP (Tir-cytoskeleton coupling protein), located within the prophage CP-933U, is an effector protein that triggers actin polymerization and is essential for recruitment of $\alpha$ actinin and A/E lesion formation (Campellone et al., 2004). Once translocated through the TTSS, TccP binds indirectly with Tir and then activates the neuronal Wiskott-Aldrich syndrome protein (N-WASP) and is able to then stimulate Nck-independent actin polymerization leading to pedestal formation (Carlier et al., 2003). 


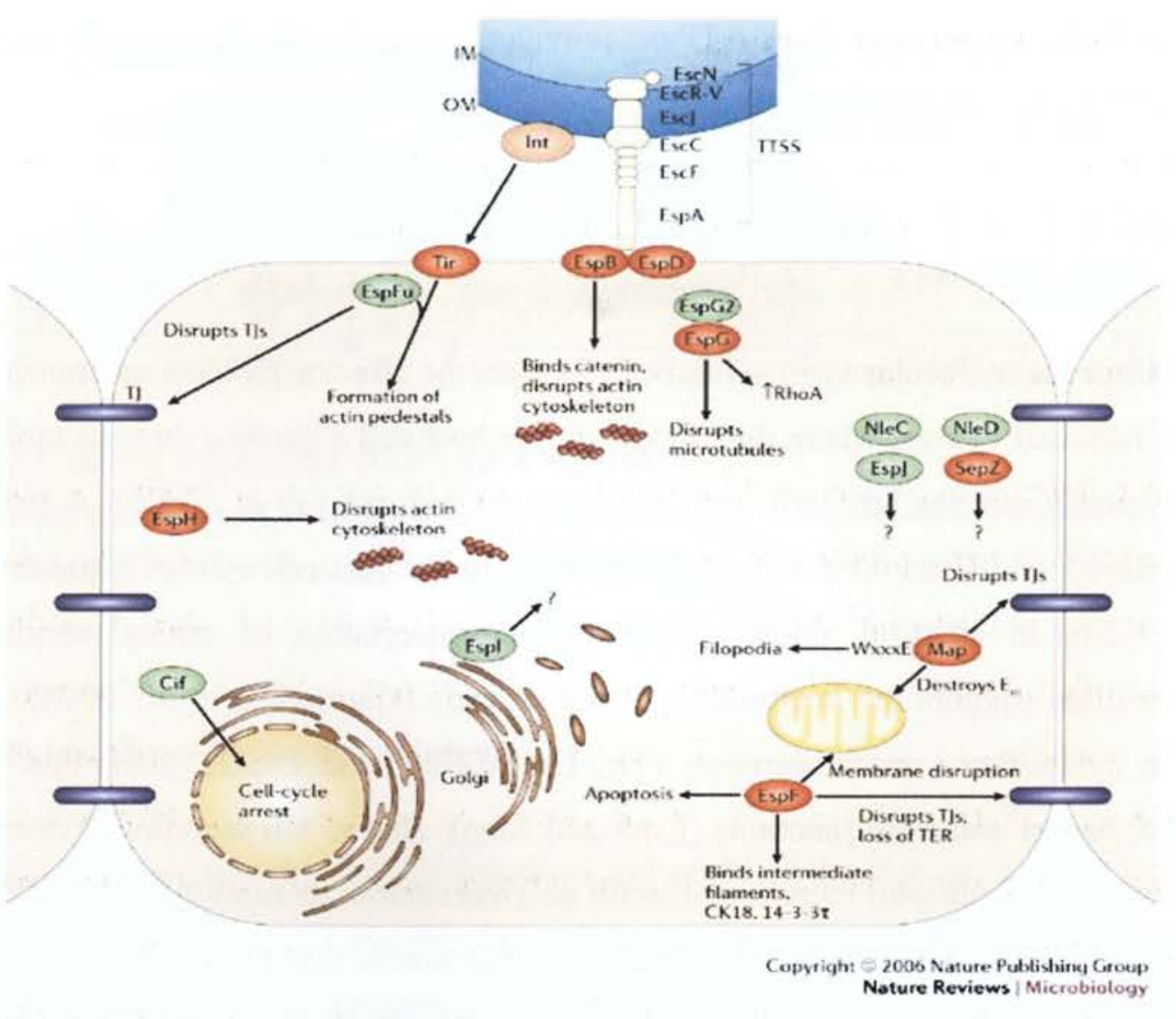

Figure 1.2-6 Simplified cartoon model for EHEC translocation of LEE encoded and other proteins into the host cell cytosol.

EspA filaments of the TTSSs form a needle complex that spans the bacterial inner and outer membrane (IM and $\mathrm{OM}$ ). Various effector proteins (Esps), including Tir, are translocated into the host cell via the TTSS. Intimate adhesion of the bacteria to the host cell is mediated by the binding of the bacterial adhesin intimin to its receptor Tir. Translocated effector proteins trigger cytoskeleton rearrangements (Tir, EspH, EspF, EspG), disruption of the epithelial barrier (EspF and Map), cytotoxicity (EspF and Cif), and host cell responses that ultimately generate watery diarrhea. EHEC Tir is not tyrosine phosphorylated and does not interact with Nck in order to trigger actin polymerization at the site of bacterial adhesion; instead, EHEC translocates an effector protein, TccP/EspFu, which has an Nck-like activity and is essential to promote the reorganization of the actin cytoskeleton underneath adherent EHEC bacteria. Reproduced from Hayward $e t$ al., 2006 with the permission of Nature Reviews Microbiology, Nature Publishing Group. 


\subsubsection{EHEC Adherence Factors}

To date, a number of adhesion factors, both fimbrial and non-fimbrial, have been suggested to play a role in EHEC host cell adhesion (Torres et al., 2005a).

\subsubsection{Fimbrial Adhesins}

Since intimin mutants are still able to colonize host cell epithelial cells, it has been suggested that bacteria produce other yet unidentified adhesins (Torres et al., 2003). To date, several fimbriae implicated in the binding of EHEC to host intestinal cells have been identified. Torres et al. (2004) have characterized 2 loci-encoding long polar fimbriae which have been proposed to mediate bacterial adherence to tissue culture cells (Torres et al., 2004). EHEC mutants in $l p f$ showed reduced adherence to epithelial cells and formed fewer microcolonies than the wilde type (Torres et al., 2004). The expression of $l p f$ was observed in response to environmental signals, such as temperature $\left(37^{\circ} \mathrm{C}\right), \mathrm{pH}$ ( $\mathrm{pH}$ 6.5), and maximal expression occurred only during the late exponential phase of growth (Torres et al., 2002; Torres et al., 2004). In addition, a cluster of $s f p$ genes present only on the large plasmid of sorbitol-fermenting EHEC (pSFO157) has been suggested to mediate mannose-resitant hemagglutination and be sufficient in the expression of a novel type of Sfp fimbria (Brunder et al., 2001). Other reports have implicated curli (Kim \& Kim, 2004), a type I pilus homolog (F9) (Low et al., 2006), and a type IV pilus (TFP) in LEE-negative non-O157: H7 STEC (Srimanote et al., 2002) as mediators of adherence. Recent data demonstrating a role for a fimbrial factor in the adherence of EHEC were published by Xicohtencatl-Cortes et al. (2007). They propose that EHEC produces adhesive type IV pili, termed HCP (hemorrahagic coli pilus), that form bridges between bacteria adhering to human and bovine host cells. Since the expression of these putative adhesins was observed only under specific environmental conditions, additional experiments are required to elucidate the detailed mechanisms of colonization of the gastrointestinal tract of human and bovine organisms (XicohtencatlCortes et al., 2007). 


\subsubsection{Nonfimbrial Adhesins}

Intimin is a key adhesion factor demonstrated to play a role in EHEC adherence to intestinal epithelial cells (McKee et al., 1995; Sinclair \& O'Brien, 2002). McKee et al. (1995) has shown that mutants defective in the E. coli O157:H7 eae (Escherichia coli attaching and effacing) gene were unable to adhere to epithelial cells, however when intimin was reintroduced, the phenotype was fully restored.They also provided evidence that eae introduced in either $\mathrm{pEB} 310$ or $\mathrm{pEB} 311$ was not sufficient to confer adherence on $\mathrm{K}-12$ strains, resulting in a similar phenotype of impaired microcolony formation in HEp-2 cells. Their data together with those of Dytoc et al. (1993) and Jerse et al. (1991) and suggest that additional factors not directly involved in adherence of EHEC may aid in the localization of intimin by the bacteria.

In addition to intimin, several nonfimbrial surface proteins have been proposed as novel adhesion factors including Iha (adherence conferring molecule), OmpA (Outer membrane protein A), Saa (autoagglutinating adhesion), ToxB, Efa-1 (EHEC factor for adherence) (Nicholls et al., 2000; Tarr et al., 2000; Paton et al., 2001; Stevens et al., 2002).

As described above, intimin is an outer membrane protein adhesin, which recognizes and binds to its translocated intimin receptor (Tir) to mediate intimate attachment. Intimin, a 94-97 $\mathrm{kDa}$ protein, is encoded by the eae gene within the LEE (Elhanafi et al., 2004). It is exported to the periplasm via the general secretory pathway and inserted into the outer membrane where it can interact with its own injected receptor or bind to host cell integrins and nucleolin (Sinclair \& O'Brien, 2002; Garmendia et al., 2005). Intimin has two functional regions, a highly conserved $\mathrm{N}$-terminal region and a highly variable C-terminal region which defines the types of intimin (Touzé et al., 2004). Intimin is classified as follows; types $\alpha, \beta, \gamma, \delta$, and $\varepsilon$ (Sinclair and O'Brien 2004). Intimin types $\varepsilon$ and $\gamma$ are present in EHEC strains, whereas type $\alpha$ is mainly associated with EPEC (Caprioli et al., 2005). Intimin B is found in EPEC and EHEC and is associated with rabbit Peyers's patch lymphid follicles (Caprioli et al., 2005). EHEC serotype O157:H7 produce $\gamma$ intimin (Caprioli et al., 2005). 
Intimin is composed of two monomeric subunits, each monomer consisting of a transmembrane region and an extracellular rod formed by three immunoglobulin-like domains (D0 to D2) and one lectin-like domain (D3) (Adams et al., 2005). The Nterminal region of intimin is inserted into the bacterial outer membrane, forms a B-barrellike structure and mediates dimerization, whereas the $\mathrm{C}$-terminal region of intimin extends from the bacterium, and interacts with receptors in the host cell plasma membrane (Adams et al., 2005). Each type of intimin binds to Tir to promote tight adherence of the organism to the host-cell plasma membrane (Sinclair \& O'Brien, 2002). $\gamma$ intimin also binds to nucleolin (host cell receptor) (Sinclair \& O'Brien, 2002). This interaction has been shown to promote initial adherence to the host cells (Campellone \& Leong, 2003). Once Tir has been translocated into the host cell, it moves to the plasma membrane where it forms a hairpin loop structure. The cytosolic domains of Tir interact with cytoskeleton proteins which lead to formation of actin pedestal structures (Garmendia et al., 2005).

Besides intimin, a number of other adhesins have been suggested to play a role in EHEC host cell adhesion. E. coli factor for adherence (Efa) has recently been proposed to contribute to adherence of $\mathrm{O} 157: \mathrm{H} 7$ to Chinese hamster ovary cells. Mutants defective in this non-fimbrial factor showed a significant decrease in adherence to bovine intestinal epithelium and human tissue cultures relative to the parent strain (Stevens et al., 2002; Badea et al., 2003). Since serotype EHEC O157:H7 strains (EDL933 and O157Sakai) have a truncated form of $e f a-1$, it has been proposed that this gene has a role in bacterial adhesion and intestinal colonization (Nicholls et al., 2000). Interestingly, efa-l has a significant homology to the tox $\mathrm{B}$ gene which has been associated with adherence to epithelial cells (Nicholls et al., 2000). Stevens et al. (2004) have shown that mutants defective in the E. coli $\mathrm{O} 157: \mathrm{H} 7$ toxB gene resulted in lower expression and secretion of type III secreted proteins and decreased microcolony formation on epithelial cells. They also provided evidence that a mutation in the truncated efa- 1 resulted in a similar phenotype of impaired microcolony formation on HeLa cells. Their data together with those of Tatsuno et al. (2001) suggest that both genes may function to influence the expression of TTSS at a posttranscriptional level (Tatsuno et al., 2001). 
The non-fimbrial Iha (IrgA homologue adhesion) has also been reported to play a role in EHEC host cell adhesion. Tha is a $67 \mathrm{kDa}$ protein found in EHEC strains and is an outer membrane protein which confers adherence to non-adherent laboratory strains (Tarr et al., 2000). Tarr et al., (2000) proposed that Iha mediates adherence to HeLa cells only when it is expressed in non-fimbriated E. coli strain. Probably K-12 strains that lack intimin are able to use Tha for adherence and colonization.

Outer membrane protein $\mathrm{A}(\mathrm{OmpA})$ has also been shown to act as an E. coli O157:H7 adhesin, playing a role in bacterial adherence to HeLa and Caco-2 cells (Torres \& Kaper, 2003). Various other adhesins including long polar fimbriae (Torres et al., 2004), curli (Uhlich et al., 2001) and F9 fimbriae (Dziva et al., 2004; Low et al., 2006) have been implicated as mediators of adherence with no apparent pathogenic role. However, the expression of these described putative adhesins is under tight regulation and thus far intimin is the only factor clearly demonstrated to play a role in EHEC adherence (Torres \& Kaper, 2003).

\subsection{8 p0157:H7 Plasmid}

E. coli $\mathrm{O} 157: \mathrm{H} 7$ carry a large plasmid (pO157), about 93-104 kB in size, which has been suggested to play a role in the pathogenesis of EHEC (Ostroff et al., 1990; Paros et al., 1993). pO157 is a dynamic structure containing mobile genetic elements such as transposons, prophages and parts of other plasmids, and as a consequence, the putative virulence factors harboured by this plasmid, appear to be unevenly distributed in EHEC O157 strains (Caprioli et al., 2005). The plasmid typically carries several virulence genes including $h l y \mathrm{~A}, k a t P, \operatorname{esp} P, \operatorname{tox} B, \operatorname{tag} A$, etcC-O , and $h l y A-D$ (Tatsuno et al., 2001). kat $P$ and $\operatorname{esp} P$ genes encode a katalase-peroxydase and a serine protease, respectively. Their role is still unclear, however studies have shown that antibodies to the espP product are present in sera from children with EHEC infection (Caprioli et al., 2005). Little is known about EspP, although it has been suggested it may mediate the cleavage and the degradation of the human coagulation factor $\mathrm{V}$, which could result in mucosal hemorrhage, common in HC (Law, 2000). ToxB may confer the ability to allow 
adherence to host-cells since strains mutated in $\operatorname{tox} B$, have been demonstrated to reduce adhesion to cultured epithelial cells (Stevens et al., 2004). Additionally, a type II secretion system has been associated with this plasmid, yet the secreted targets pertaining to this system have not been identified (Tatsuno et al., 2001; Caprioli et al., 2005). StcE, a protease, which also is present on the virulence plasmid, has been proposed to contribute to the intimate adherence of E. coli $\mathrm{O} 157: \mathrm{H} 7$ to host cells (Grys et al., 2005). The $s t c E$ gene, upregulated by Ler, is able to destroy the layer of mucins and glycoproteins, and thereby aids the bacterium to adhere to the host cells by subverting the host mucosal defenses (Grys et al., 2005).

\subsubsection{Regulation of the EHEC Virulence Genes}

In order to colonize the host, activate virulence genes, and initiate the disease process, EHEC must sense its surrounding and respond accordingly to any environmental changes (Mekalanos, 1992). Thus, the ability to respond rapidly is essential for the success of the pathogen. Appropriate expression of TTSS genes is achieved through the integration of several signal transduction systems, which allow pathogens to respond to changing microenvironments during infection (Spear et al., 2006). The complex regulatory cascades reflect their ability to adapt to different niches both inside and outside of the host.

LEE-encoded virulence factors are regulated by the LEE-encoded regulator (Ler) which are further positively and negatively modulated by GrlA and GrlR respectively (Deng et al., 2004). It has also been reported that regulatory mechanisms of activation of LEE1 require perC (plasmid encoded regulator) in EPEC and pch A, B and C (renamed perC homologue pchA, pchB and pchC) in E. coli O157:H7 (Spear et al., 2006). Although LEE1 expression in EPEC is tied to perC, however this is not the case in EHEC which possesses a number of perC chromosomal homologues that act to increase

LEE1 expression (Spear et al., 2006). LEE genes may be regulated at the posttranscriptional level (Roe et al., 2003; Stevens et al., 2004; Deng et al., 2005). Zang et al. (2004) proposed that LEE expression may be repressed by a cryptic second type III 
secretion island (Zhang et al., 2004). There is likely a cross talk between regulators from other O-island and TTS-related genes such as flagella (Zhang et al., 2004). Cell to cell signaling between bacteria (quorum sensing) is activated via the LuxS, QseA and SdiA systems (Sperandio et al., 1999; Sperandio et al., 2001; Sperandio et al., 2002); however the mechanism for SdiA binding of signaling HSL to repress EspD and intimin remains to be elucidated. Additionally recent evidence has shown that EHEC may hijack signals produced by other microbial flora and the host that lead EHEC to regulate its virulence genes in a coordinated manner (Clarke \& Sperandio, 2005). According to the model proposed by Hughes \& Sperandio (2008) EHEC sense autoinducer (AI-3), adrenaline and noradrenaline (NA) signals to activate motility genes (flhDC), Stx genes and LEE genes (components of a type III section system).

Overall, TTSS expression in EHEC is under tight regulatory control, however with considerable variation between strains that may contribute to their epidemiology in both humans and cattle (Spear et al., 2006).

\subsection{Modulation of Host Cell Function}

Multiple functions including disruption of epithelial barrier, altered ion secretion, disruption of tight junctions, neutrophil recruitment, actin polymerization, disruption of cell cycle and transcriptional changes explain the physiological outcome: watery persistent diarrhea (Garmendia et al., 2005).

\subsection{Disruption of the Barrier Function}

EHEC infection alters the barrier function through disruption of the epithelial barrier and increased monolayer permeability through alteration of tight junctions. EHEC activates several pathways contributing to the disruption of barrier function (Garmendia et al., 2005). The pathway is the redistribution and activation of ezrin by phosphorylation on threonine and tyrosine (leads to disruption of tight junctions). A second pathway is the redistribution of tight junction proteins: occludin is dephosphorylated by ser/threonine 
phosphatases and moves from tight junctions to the cytosol (Berryman et al., 1993). Another pathway is phosphorylation of myosin light chain (MLC): MLC associates with the cytoskeleton and is phosphorylated by MLC kinase - ATP hydrolysis and movement of filaments past each other to cause an increase in paracellular permeability (ManjarrezHernandez et al., 1996). A four pathway is the disruption of adherent junctions: phosphorylation of protein kinase C (PKC) which associates with cadherins dissociation of cadherin/ßcatenin complex which forms adherent junctions. Several effectors are involved in induction of these effects including EspF, EspFu/TccP and Map (Malladi et al., 2004).

\subsubsection{Factors Contributing to Diarrhea}

In addition to the disruption of the barrier function, several factors contribute to EHEC induced secretory diarrhea. Changes in the host cell electrolyte transport and decrease in membrane potential and increase in short circuit current are observed in EHEC infections. Additionally, changes in chloride secretion and bicarb-dependent chloride transport are observed (Hecht \& Koutsouris, 1999). EHEC induces tyrosine phosphorylation (Tyr-P) of phospholipase C $\gamma 1$ (PLC- $\gamma 1$ ), cleaves phophatidyl-inositol 4,5-biphosphate (PI45P2) producing 1,4,5-triphosphate (IP3) and (dyacylglycerol) DAG which activates PKC for secretion of ions and fluids (Crane et al., 1999). Recent research revealed that several ion transport and ion channel proteins are upregulated during TTSSEHEC infection (Hardwidge et al., 2004).

\subsubsection{Host Cell Cytoskeletal Rearrangements}

Initial stages of infection are characterized by filapodia of the host tissue at the site of infection that extend and sway for about $20 \mathrm{~min}$ and retract into the host cell (Map and Cdc42 dependent) (Kenny et al., 2002). These are followed by massive rearrangement of the host actin microfilaments triggering $\mathrm{A} / \mathrm{E}$ lesions and formation of pedestal like structures of host tissue under adherent bacterium $-10 \mu \mathrm{m}$ outward from host cell. EHEC 
can move across the surface of infected cells at speeds of up to $0.1 \mu \mathrm{m} / \mathrm{sec}$ (Figure 1.4-1) (Sanger et al., 1996). Y474 of EHEC Tir is phosphorylated and the Y474 containing region recruits and activates $\mathrm{N}-\mathrm{WASP}$ activating $\mathrm{Arp} 2 / 3$ and triggering actin polymerization and pedestal formation (Cantarelli et al., 2002).

Watery diarrhea may be generated by disruption of the epithelial barrier, cytoskeleton rearrangements, the inflammatory response to infection, and active chloride secretion resulting from signal transduction, whereas bloody diarrhea may be induced by verotoxin damage to small blood vessels in the large intestine (Law, 2000; Gyles, 2004 personal communication). In addition to causing cytotoxicity, Stx have also been implicated in inducing programmed cell death or apoptosis (Ceponis et al., 2003). HEp-2 cells exposed to the toxins for 24-48 hours have been shown to show classic morphologic features associated with apoptosis such as cell shrinkage, membrane blebbing, and nuclear chromatin condensation (Ceponis et al., 2005). 


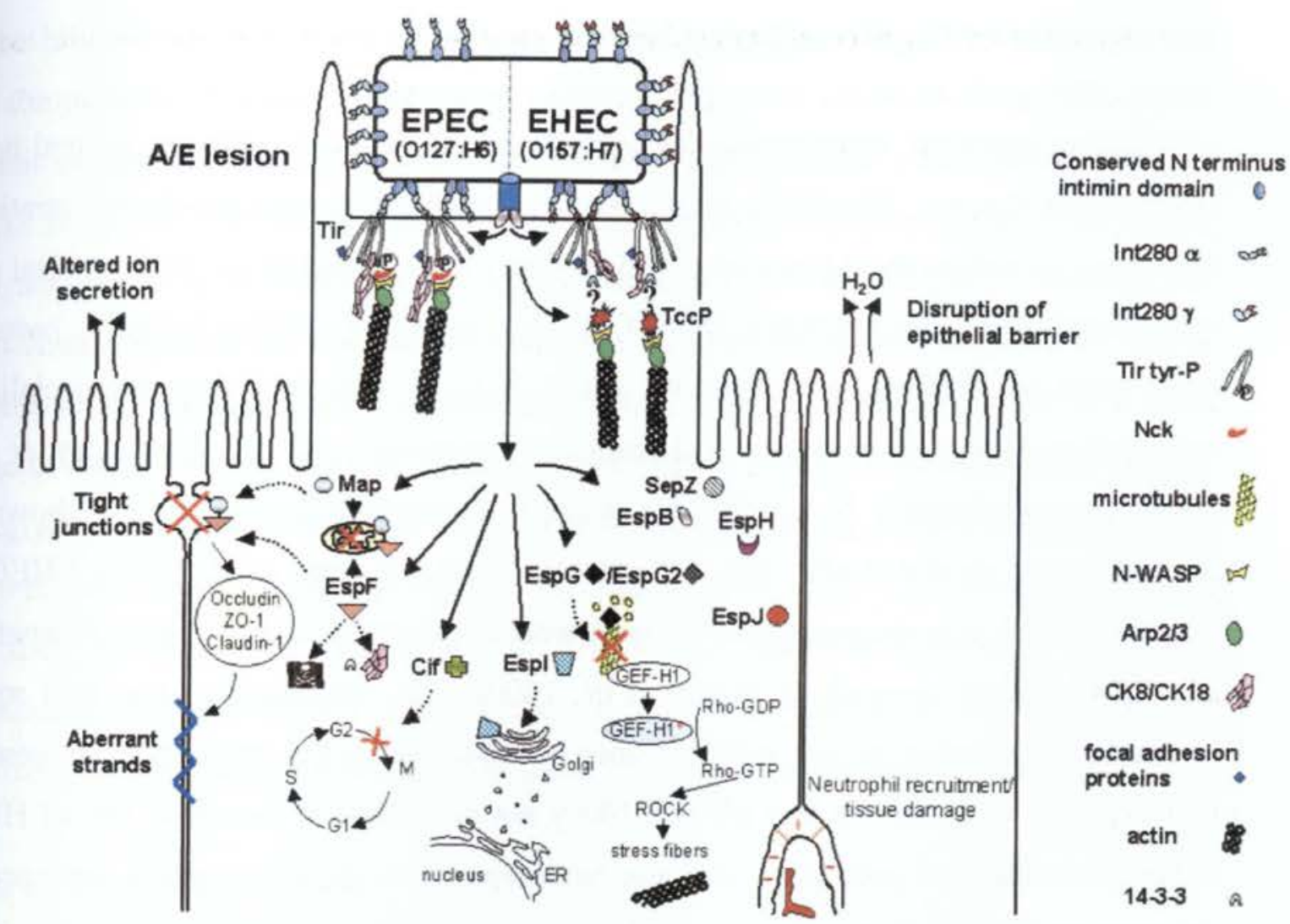

Figure 1.4-1 EPEC and EHEC translocate effector proteins into the host cell cytosol.

These effectors trigger cytoskeleton rearrangements (Tir, EspH, EspF, and EspG), disruption of the epithelial barrier (EspF and Map), cytotoxicity (EspF and Cif), and host cell responses that ultimately generate watery diarrhea. Different from EPEC Tir, EHEC Tir is not tyrosine phosphorylated and does not interact with Nck in order to trigger actin polymerization at the site of bacterial adhesion; instead, EHEC translocates an effector protein, TccP/EspFu, which has an Nck-like activity and is essential to promote the reorganization of the actin cytoskeleton underneath adherent EHEC bacteria. ER, endoplasmic reticulum. Reproduced from Garmendia et al., 2005 with the permission of Infection and Immunity, American Society for Microbiology. 


\subsection{Impact of Ingestion Stress on Virulence}

During ingestion, enteropathogens are exposed to, and must overcome several hostenvironment stresses. The ability of pathogens to withstand physiological concentrations of bile salt as well as short chain fatty acids is likely to be important for their survival and consequently for their persistence in the GI tract of their host. During infection, bacteria must adapt to changing environments and it is likely that they express specialized colonization factors in a hierarchical manner (Cummings et al., 1987; Begley et al., 2005). Enteropathogenic E. coli (EPEC), which is a related pathogen, has been shown to have enhanced virulence after bile salt and acid stress (de Jesus et al., 2005). EHEC is also well suited to overcoming acid stress in order to infect and cause disease (Elhanafi et al., 2004). Recent research by House et al., (2006), has demonstrated that acid stress increases the adhesion to host cells but does not affect verotoxin production, suggesting that exposure to extremely low $\mathrm{pH}$ will likely not contribute to increased risk of HUS. Other gastrointestinal pathogens have also been reported to show changes in virulence in the presence of bile. Ramos-Morales and coworkers (2003) have shown that synthesis of enterobacterial common antigen (ECA) contributes to Salmonella virulence by protecting the pathogen from BS. Shigella grown in BS have been shown to enhance Ipa protein secretion and increase invasion of epithelial cells (Pope et al., 1995). Vibrio parahaemolyticus has been shown to have increased expression of TDH virulence factor (Osawa et al., 2003). Contrary to the positive effect of bile on V. parahaemolyticus, Schuhmacher et al. (1999) suggested that Vibrio cholerare in the presence of bile, resulted in decreased virulence gene expression.

\subsection{Bacterial Response to Bile Salts}

\subsubsection{Bile Composition and Function}

After overcoming the acid barrier in the stomach, enteric bacteria including EHEC are exposed in the intestinal tract to bile salts. Bile is a detergent-like digestion fluid that disrupts the lipid bilayer structure of cellular membranes, induces protein mis-folding and cause oxidative damage to DNA (Begley et al., 2005). In humans, the major components 
of the bile include bile acids, cholesterol, phosholipids (primarily phosphatidylcholine) and the pigment biliverdin (Carey et al., 1994). Bile acids represent about $50 \%$ of the organic components of bile and are conjugated as $\mathrm{N}$-acyl amidates with glycine or taurine before secretion. The carboxyl group of the bile acid and the amino group of the amino acid are linked by an amide bond (peptide linkage) (Hofmann, 1999). Amidation has significant effects on the bile acid solubility since the unconjugated bile acids are hardly soluble at physiological $\mathrm{pH}$. Conjugation allows bile acids to be soluble over a wide range of ionic strengths, calcium concentrations and $\mathrm{pH}$ values (Hofmann, 1999). Taurine and glycine conjugates are called bile salts since they are totally ionized at physiological $\mathrm{pH}$ while the unconjugated bile salts are not (Begley et al., 2005). The major BS present in the intestine are deoxycholate (NaDOC), chenodeoxycholate (NaCDC), ursodeoxycholate (NaUDC) and glycholate (NaGIC) (Gabig et al., 2002). The approximate concentrations entering the small intestine are presented in Table 1-1:

\section{Table 1-1 Bile Salts in Duodenum}

\begin{tabular}{|l|l|}
\hline Bile salt & Duodenum percent of bile salts \\
\hline NaGIC & $31.1^{\mathrm{a}}$ \\
\hline $\mathrm{NaUDC}$ & 0.8 \\
\hline $\mathrm{NaCDC}$ & 35.7 \\
\hline $\mathrm{NaDOC}$ & 14.4 \\
\hline
\end{tabular}

A litre of bile, which is a 'biological detergent', passing through the duodenum each day represents a serious challenge to intestinal pathogens and thus forms an important part of the body's defense system (Hofmann, 1999).

\footnotetext{
${ }^{a}$ from ratio of glycine: taurine amidates and percent cholate (Bernstein et al., 1999).
} 


\subsubsection{Antimicrobial Actions of Bile}

The main antimicrobial action of bile has been demonstrated to be through damage to cell membranes by altering membrane integrity/permeability (Pazzi et al., 1997). There are several important factors that determine the exact outcome of bile on cell membranes (Begley et al., 2005). The concentration of bile is a major important factor. High levels of BS have been shown to dissolve membrane lipids and dissociate membrane proteins, causing disruption of membrane integrity (Coleman et al., 1980). BS at low concentrations can also affect the physical chemical properties of cell surfaces. The type and structure of bile also may determine the outcome of bile action on the cell membrane (Begley et al., 2005). For example, bovine bile which passively diffuses much more slowly across the lipid bilayer than porcine bile is also less inhibitory (Legrand-Defretin et al., 1991). Although the composition of porcine bile is more similar to that of human bile, bovine bile is commonly chosen to assess the in vitro bile tolerance of bacteria (Begley et al., 2005). The membrane architecture and composition are also key factors; charge, hydrophobicity and lipid fluidity have been shown to have significant consequences. Chou and Cheng, (2000) observed that freezing significantly damages the cell membrane lipopolysaccharides making them more susceptible to BS. Besides membrane damage, bile has been shown to induce DNA damage, alter conformation of proteins (misfolding or denaturation) and cause oxidative stress. Research by Bernstein et al., (1999) has shown that BS induce the expression of several stress response genes: micF, osm Y and $\operatorname{din} D$.

\subsubsection{Bile Tolerance}

Bile tolerance is poorly understood, particularly in Gram negative bacteria, however it is believed that they are more resistant to bile than Gram positive bacteria (Begley et al., 2005). Prouty et al. (2004) showed that Salmonella is capable of colonizing the gall bladder where bile is in extremely high concentrations (Prouty et al., 2004). E. coli is also considered to be very bile resistant since it has been isolated from gallbladder and 
bile of animals and humans (Brook, 1989; Cable et al., 1997; Carpenter, 1998; Flores et al., 2003). Another study by Gänzle et al. (1999) reported that E. coli may inhabit the small intestine of a gastrointestinal model in the presence of high concentrations of porcine bile extract whereas Listeria was rapidly killed. Helicobacter and Campylobacter are also bile resistant and have been isolated from bile ducts, liver and gallbladder (Fox et al., 1995). In contrast, $H$. pylory has been shown to be sensitive to bile which may explain why it has never been observed in faecal samples or cholecystectomy specimens (Begley et al., 2005).

\subsubsection{Genetics of Bacterial Bile Tolerance}

The pathogenic strains of $E$. coli are able to survive the BS stress by developing adaptive strategies to the stress, including: drug efflux pumps (AcrAB pump), porin proteins (OmpF, OmpC), transport proteins, murein lipoproteins, peptidoglycan associated lipoproteins (Gunn, 2000). Research by Bernstein et al. (1999) has shown that BS induces the expression of several stress response genes: $m i c F$, osm $Y$ and din. Another study by Wang (2002) suggested that the outer membrane protein OmpA plays a major structural role in E. coli membrane stability. E. coli $\mathrm{K} 1 \mathrm{ompA}$ mutants are significantly more sensitive to environmental stresses (cholate, acidic environment and high osmolarity) resulting in reduced stress resistance. $\mathrm{OmpF}$ and $\mathrm{OmpC}$, two major outer membrane proteins, are responsible for the passive diffusion of small molecules in the periplasm. Their expression is conversely regulated; because the OmpF porins form channels larger than those of OmpC. It has been reported that OmpC can exclude larger molecules more efficiently than OmpF porins (Bernstein et al., 1999).

In addition, several other factors have been demonstrated to contribute to bacterial resistance to environmental stress. Among these are colanic acid (CA) exopolysaccharides (EPS) which are cell surface structures and that provide the first line of defence for bacterial cells (Mao et al., 2006). According to these studies, CA EPS of E. coli $\mathrm{O} 157: \mathrm{H} 7$ provides a protective barrier to the pathogen while they are exposed to the various stress conditions in the gastrointestinal tract. The resistance of Vibrio 
cholerae and other Vibrio species to BS has been attributed to the lipopolysaccharide (LPS) and outer membrane porins (Provenzano et al., 2000).

\subsubsection{Bile Sensing and Regulation of Bile Stress Response}

The mechanisms used by bacteria to sense and respond to bile are poorly understood. Bile is thought to be detected by a two-component system (TCSs) (Parkinson and Kofoid, 1992). TCSs are composed of a membrane-associated histidine kinase and a cytoplasmic response regulator. The histidine kinase detects the presence of bile, it transduces the signal to the response regulator which instructs the cell to respond to the change via changes in the expression of specific virulence factors (Parkinson and Kofoid, 1992). Bile may also be sensed by responding to the disruption of membrane integrity. Bacterial membrane damage sensors such as E. coli $\mathrm{Cpx}$ and $\mathrm{RcsCb}$, which sense alterations in the cell surface, may function as sensors and signal transducers (De Wulf et al., 2002; DiGiuseppe and Silhavy, 2003). In addition, the accumulation of proteins damaged or denatured by bile may activate a general stress response. Transcription of stress genes with significant roles in bile resistance has been shown to be controlled by two well characterized transcriptional regulators: Sigma B $\left(\sigma^{\mathrm{B}}\right)$ and $\mathrm{RpoS}\left(\sigma^{\mathrm{S}}\right)($ Cheville et al., 1996; Ferreira et al., 2001).

\subsubsection{Bile and Pathogenesis}

Continuous monitoring of environmental changes is essential for survival for enteric bacteria when making the transition to the human host. It is not surprising that enteric pathogens use bile as an environmental cue to establish the organism's intestinal location and co-ordinately modulate the expression of virulence factors. It is likely that some gene products expressed in bile tolerance assist in survival and colonization of the intestinal tract and thus in their own right, function as virulence factors (Begley et al., 2005). Recent work has uncovered the pathogen responses to bile that alter the expression of virulence factors in some of the GI pathogens including Salmonella, 
Shigella, Vibrio, Campylobacter and enteropathogenic E. coli (Pope et al., 1995; Prouty \& Gunn, 2000; Osawa et al., 2002; Prouty et al., 2004; de Jesus et al., 2005; Torres et al., 2007).

Pathogens respond to bile in different fashions in order to facilitate their own pathogenic mechanisms (Begley et al., 2005). Prouty et al. (2000, 2004) demonstrated that Salmonella sense the bile as environmental signal which consequently downregulates invasion in the intestinal lumen where there are high concentrations of bile. However, upon transit of the mucus layer, where bile concentrations decrease, the expression of the invasion machinery is initiated resulting in an increase in SP-1 genes involved in epithelial cell entry. Pope et al. (1995) provide evidence that Shigella $(S$. flexneri and $S$. dysenteria) respond to bile in a contradictory fashion; Ipa protein secretion is up-regulated, and invasion of epithelial cells is increased due to enhanced adherence . A study by Osawa et al. (2006) on Vibrio parahemolyticus showed that bile acids enhance production of the virulence factor thermostable direct hemolysin (TDH). In addition to these findings, another study by Pace et al. (1997) also demonstrated that bile acids favor growth of $V$. parahaemolyticus and enhance adherence to epithelial cells. Contrary to the positive effect of bile to $V$. parahaemolyticus, two independent studies demonstrated that growth of $V$. cholerae in bile results in decreased virulence expression (Gupta and Chowdhury, 1997; Schuhmacher and Klose, 1999). Growth in the presence of bile resulted in a decrease in cholera toxin (CT) production and toxin-coregulated pilus (TCP) but an increase in motility. Another study reported that bile inactivates the transcription factor ToxT which induces numerous virulence promotors including those for CT and TCP (Schuhmacher and Klose, 1999). The model proposed by these authors suggests that once bacteria swim into the mucosal gel where bile concentrations are low, motility is decreased, ToxT activity is restored and virulence gene expression required for colonization and disease is induced (Schuhmacher \& Klose, 1999).

The role of bile in EPEC pathogenesis has also been identified. De Jesus et al. (2005) identified profound changes in host adhesion of EPEC after exposure to BS stress. BS stress of EPEC significantly increases the adhesion of surviving EPEC to epithelial cells compared to unshocked EPEC. The authors also observed a slight increase in flagella expression and bundle-forming pilus (BFP) after BS (de Jesus et al., 2005). To 
our knowledge there are no studies on the impact of BS on changes in E. coli 0157:07 virulence factor expression.

\subsection{Short Chain Fatty Acid Stress Response}

\subsubsection{Short Chain Fatty Acid Composition and Function}

Transiting to the large intestine, EHEC must also resist the stress of exposure to short chain fatty acids (SCFA) which are key end products of colonic fermentation (Arnold et al., 2001; Polen et al., 2003). Although the pH of the intesine is almost neutral, there are high concentrations of SCFA present in the large intestine, mainly acetate, propionate and butyrate (Cummings et al., 1987). The organic acids are preferentially absorbed across the epithelial membranes in the human ileum and colon. Butyrate is of particular importance because it is the major source of energy for colonic epithelial cells (Borthakur et al., 2006). Propionate is largely taken up by the liver whereas acetate enters the peripheral circulation to be metabolized by peripheral tissues (Puchowicz et al., 1999). The concentration of SCFAs is estimated to be low, between 20 and $40 \mathrm{mM}$ total SCFAs in the ileum, and ranges from 100-300 $\mathrm{mM}$ in the colon, depending on species and diet (Cummings et al., 1987; Macfarlane et al., 1992). Because the protonated form of SCFA equilibrates across the cytoplasmic membrane, SCFA can lower internal pH even when the external pH is neutral (Roe et al., 1998). The $\mathrm{pH}$ of the colon and ileum are similar at around 6.7 (Lawhon et al., 2002). 


\subsubsection{Short Chain Fatty Acid Tolerance and Pathogenesis}

Exposure to SCFAs is considered a stress condition and likely impacts the survival of microorganisms, evidenced by the fact that they are widely used as food preservatives to prevent the growth of bacteria (Abdul-Raouf et al., 1993). A study by Lasko et al. (2000) investigated the response of several probiotics and E. coli K12 after exposure to varying acetate challenges. The authors pointed out that capacity of nonpathogenic E. coli to tolerate acetate was dependent on the carbon source. E. coli exibits higher acetate tolerance in the glycerol grown cultures compared to glucose supplemented media. Moreover, E.coli grown the presence of glycerol is almost tolerant to acetate as are the acetic acid bacteria $(\mathrm{AAB})$ which are best known to be tolerant to acetate (Lasko et al., 2000). Another study by Polen et al. (2003) investigated gene expression patterns E. coli $\mathrm{O} 157: \mathrm{H} 7$ due to the presence of acetate. The adaptive response of nonpathogenic E. coli exposed to SCFA (acetate and propionate) has been shown to include an increased expression of chemotaxis and flagellum genes, both of which can play a role in colonization of the gastrointestinal tract (Polen et al., 2003). Lawhon et al. (2002) demonstrated that SCFAs alter the expression of Salmonella typhimurium invasion genes. Acetate activated S. typhimurium invasion whereas propionate and butyrate (primarly found in the colon) had the opposite effect. The authors suggested that the composition of SCFAs in the small intestine provides a cue to activate Salmonella invasion while that in the large intestine inhibits invasion (Lawhon et al., 2002). Consistent with these findings, Gantois et al. (2006) observed that most genes significantly downregulated after exposure with butyrate are genes of the pathogenicity island 1. Another study by Kirkpatrick et al. (2001), using a proteomic approach, has revealed that $E$. coli grown with acetate increased the expression of several amino acid and peptide transporters. The authors suggested that these transporters might enhance the uptake of alternative energy source if the cells perceive acetate uptake as a sign of decreasing availability of carbohydrates. Accentuated expression of components of the RpoS regulon (stress response system) was observed after short term exposure with acetate (Kirkpatrick et al., 2001). 


\subsection{Stress and EHEC Virulence}

While significant advances have been made in EHEC pathogenesis, we still do not fully understand the impact of environmental stress on the virulence properties of EHEC including adhesion to host endothelial cells and production of Shiga toxins. Environmental stresses have been clearly demonstrated to enhance the virulence of many enteropathogens. Recent research by de Jesus et al. (2005) has identified profound changes in host adhesion of a related pathogenic EPEC after exposure to major components of bile. BS stress of EPEC also resulted in a significantly increased adhesion of surviving bacteria to epithelial cells. In addition, our laboratory has also shown that acid shock enhances the adhesion of E. coli $\mathrm{O} 157: \mathrm{H} 7$ and its virulence (House et al., 2006). The role of bile on EHEC adhesion to host endothelial cells and toxin production has yet to be described.

The effect of SCFA on E. coli O157:H7 virulence is not well understood. Arnold et al. (2001) is one of the first reports that investigated the impact of SCFAs on E. col. This group only reported gene expression changes of $E$. coli $\mathrm{O} 157: \mathrm{H} 7$ which was grown with inhibitory concentrations of $100 \mathrm{mM}$ acetate for $30 \mathrm{~min}$. Their results provided evidence that acetate increases expression of a member of RpoS regulon, as well as various transporters and metabolic enzymes. Another study by Tao et al. (1999) has shown that E. coli grown on rich media have elevated expression of the RpoS regulon as well as OmpC and TpiA, the authors pointing out a role of glucose in generating high acetate levels. A second study by $\mathrm{Oh}$ et al. (2002) investigated the transcript profile of $E$. coli grown also in acetate as the sole carbon source and compared it with that one of the culture grown in glucose. When grown in the poor carbon source, genes involved in cell replication, transcription and translation were down-regulated whereas metabolic genes were up-regulated. Other studies also revealed increased expression of general stress response genes after short exposure of $E$. coli to high concentrations of acetate. Louise $e t$ al. (1995) have established the role of butyrate on Shiga toxin binding, internalization, intracellular transport and activation in eukaryotic cells. They suggest that butyrate may increase the sensitivity and intracellular traffic in HUVEC as well as Shiga toxin binding to the cell surface (Louise et al., 1995). Interestingly, recent research by Borthakur et al. 
(2006) showed that enteropathogenic E. coli which is a related EHEC pathogen, modulates the butyrate uptake in Caco2 cells. EPEC infection inhibits butyrate uptake in Caco2 cells whereas infection with nonpathogenic E. coli had no effect. Other complementary studies have also demonstrated that butyrate is implicated in suppressing mucosal inflammation (Inan et al., 2000; Segain et al., 2000). Taken together, these results suggest that decreased butyrate uptake caused by EPEC infection may compromise the colonic epithelial integrity, resulting in inflammation of the epithelium (Borthakur et al., 2006).

Recent studies by Carey et al. (2008) used several probiotic strains to evaluate the effect of SCFAs on Stx2 gene expression in E. coli O157:H7. All strains tested down regulated st $x 2 \mathrm{~A}$ expression to different degrees, the authors suggesting that may be attributed to the type and amount of organic acid produced by each probiotic strain. Takahashi et al. (2004) also suggested that due to butyric acid production, Clostridium butyricum might be able to inhibit EHEC growth. However further research is needed to fully understand the impact of SCFA stress on EHEC toxin production since these published reports have only examined the impact of probiotic strains. The role of major SCFA including acetate, propionate and butyrate as well as that the mixture mimicking the large intestine, on E. coli $\mathrm{O} 157: \mathrm{O} 7$ needs to be explored.

\subsection{Purpose of Investigation and Hypothesis}

\subsubsection{Research Rationale}

It is becoming increasingly clear that pathogens respond to BS and SCFA in a variety of fashions to facilitate their specific pathogenic mechanisms. Bile represses Salmonella invasion but stimulates invasion of Shigella (Begley et al., 2005). It is evident that that BS and SCFA can trigger changes in virulence properties of gastrointestinal pathogens. However, there is little known about how these stresses modulate the virulence potential of $E$. coli $\mathrm{O} 157: \mathrm{H} 7$. Further research is required to fully delineate the effect of BS and SCFA stress on EHEC virulence gene expression. This study will contribute to the developing picture of a complex relationship between E. coli $\mathrm{O} 157: \mathrm{H} 7$ 
and its environment within the host during infection. Moreover, it will be essential for describing novel approaches to prevent and heal EHEC infections.

\subsubsection{Hypothesis}

Exposure to physiological concentrations of BS and SCFA will significantly increase EHEC virulence including host cell adhesion and toxin production

\subsubsection{The Main Objectives of this Thesis}

1) Assess impact of exposure of E. coli $\mathrm{H} 157: \mathrm{O} 7$ to physiological relevant concentrations of BS on virulence including survival, host adhesion and toxin production

2) Assess impact of exposure of E. coli $\mathrm{H157:07}$ to physiologically relevant concentrations of SCFA on virulence, including host adhesion and toxin production

3) Clarify the role of known adhesins in the adhesion of BS and SCFA treated $E$ coli O157:H7. 


\section{Materials and Methods}

\subsection{Bacterial Cultivation}

Table 2.1 below details the bacterial strains utilized in this thesis project.

Briefly, the Enterohaemorrhagic E. coli $\mathrm{O} 157: \mathrm{H} 7$ strains analyzed in this study were,

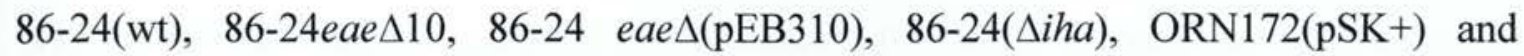
ORN172(pIHA). Bacteria were maintained as glycerol stocks and stored at $-80^{\circ} \mathrm{C}$, were routinely streaked onto Luria-Bertani (LB) agar plates (tryptone, $1 \%(\mathrm{w} / \mathrm{v})$; yeast, $(0.5 \%$ $(\mathrm{w} / \mathrm{v})$; sodium chloride, $1 \%(\mathrm{w} / \mathrm{v})$; agar $1.5 \%(\mathrm{w} / \mathrm{v})$ with or without addition of appropriate antibiotics and grown at $37^{\circ} \mathrm{C}$. The mutants were checked to make sure they retained their characteristics. Strains were stored at $4{ }^{\circ} \mathrm{C}$ and subcultured every three weeks (Riordan et al., 2000). The cultures innoculated from the LB plate were grown overnight under standardized conditions (shaking incubator at $37^{\circ} \mathrm{C}$ for 16 to $18 \mathrm{~h}$ at 200 rpm). For strain 86-24 eae $\Delta$ (pEB310), ampicillin (Sigma) was added to all plates and liquid media to a final concentration of $100 \mathrm{ug} / \mathrm{mL}$ of. $200 \mathrm{ug} / \mathrm{ml}$ Ampicillin (Sigma) was added for ORN172(pSK + ) and ORN172(pIHA) strains and 20ug/ml nalidixic acid ( Sigma) for 86-24( $\Delta i h a)$.

Table 2-1 Bacterial Strains Used in this Study

\begin{tabular}{|c|c|c|c|c|c|}
\hline Strains & Relevant characteristics & $L E E$ & VT1 & $V T 2$ & $\begin{array}{l}\text { Sourcel } \\
\text { Reference }\end{array}$ \\
\hline $86-24$ & Clinical Isolate O157:H7 & + & - & + & $\begin{array}{c}\text { (Gansheroff } e t \\
\text { al., 1999) }\end{array}$ \\
\hline $86-24$ eae $\Delta 10$ & $\begin{array}{l}86-24 \text { with deletion of eaeA } \\
\text { gene encoding intimin protein } \\
\text { required for attachment }\end{array}$ & + & - & + & $\begin{array}{c}\text { (McKee et al., } \\
1995)\end{array}$ \\
\hline $\begin{array}{l}86-24 \\
e a e \Delta(\mathrm{pEB} 310)\end{array}$ & $\begin{array}{l}86-24 \text { carrying plasmid pEB310, } \\
\text { wild-type activity being restored }\end{array}$ & + & - & + & $\begin{array}{c}\text { (McKee et al., } \\
1995)\end{array}$ \\
\hline $86-24(\Delta i h a)$ & $\begin{array}{l}\text { 86- } 24 \text { with deletion of iha gene } \\
\text { encoding iha adherence- } \\
\text { conferring protein }\end{array}$ & + & - & + & $\begin{array}{l}\text { (Tarr et al., } \\
\text { 2000) }\end{array}$ \\
\hline ORN172(pSK +$)$ & $\begin{array}{l}\text { Nonadherent laboratory } \\
\text { E. coli }\end{array}$ & - & - & - & $\begin{array}{l}\text { (Tarr et al., } \\
2000)\end{array}$ \\
\hline ORN172(pIHA) & $\begin{array}{l}\text { Nonadherent laboratory } \\
E \text {. coli carrying plasmid pIHA } \\
\text { for adherence phenotype }\end{array}$ & - & - & - & $\begin{array}{l}\text { (Tarr et al., } \\
\text { 2000) }\end{array}$ \\
\hline
\end{tabular}




\subsection{Epithelial Cell Culture}

Human epithelial cell lines, HEp-2 (laryngeal carcinoma cells), and $\mathrm{CaCo-2}$ (colonic adenocarcinoma cells) were obtained from the American Type Culture Collection (ATCC) and were used in various adhesion assays. Vero cells (African Green Monkey Kidney cells) were kindly provided by Dr. Lingwood (Hospital of Sick Children, Toronto) and were used in Vero Cell Cytotoxicity Assays (see Table 2.2 below). All tissue cell culture conditions consisted of growing the cell lines in minimal essential media 1x Eagle's (modified) (Wisent; with Earle's salts and L-glutamine) supplemented with gentamycin $(20,000 \mathrm{ug} / \mathrm{mL})$ and $10 \%(\mathrm{v} / \mathrm{v})$ heat-inactivated fetal bovine serum (FBS) (Wisent) for HEp-2 and Vero cell lines and 20\% (v/v) for CaCo-2 cells (MEM with additives). All cell lines were grown to confluence at $37^{\circ} \mathrm{C}$ and $5 \% \mathrm{CO}_{2}(\mathrm{v} / \mathrm{v})$ in cell culture vented flasks. Vero and HEp- 2 cells were maintained by subculturing the cells into fresh pre-warmed media every 3-4 days. Maintenance of the $\mathrm{CaCo}-2$ cell line required fresh media after the $4^{\text {th }}$ day of incubation and on the $6-7^{\text {th }}$ day when the cells were transfered to fresh prewarmed media. One day prior to adhesion assay, cells were seeded into 24 well or 6 well plates and grown to $80-90 \%$ confluence.

Table 2-2 Cell Lines Used in This Study

\begin{tabular}{|l|l|l|l|l|}
\hline Cell Line (ATTC\#) & Morphology & Species & Tissue/Tumor & Source \\
\hline HEp-2-(CCL23) & Epithelial & Human & Larynx, carcinoma & ATCC \\
\hline CaCo-2 -(HTB37) & Epithelial & Human & $\begin{array}{l}\text { Colon, } \\
\text { adenocarcinoma }\end{array}$ & ATCC \\
\hline Vero-(CCL 81) & Fibroblast & $\begin{array}{l}\text { African } \\
\text { Green } \\
\text { Monkey }\end{array}$ & Kidney & $\begin{array}{l}\text { Hospital for Sick } \\
\text { Kids (Lingwood } \\
\text { Lab) }\end{array}$ \\
\hline
\end{tabular}

\subsection{Bile Salt Treatment of $E$. coli $0157: \mathrm{H7}$}

One day prior to the experimental day, bacteria (Table 2.1) were grown in LB broth supplemented with appropriate antibiotics when necessary. A 1:8 dilution of overnight culture was made into prewarmed Dulbecco's MEM (DMEM) pH 7.4 (Wisent; 4.5g/L 
glucose, L-glutamine, sodium pyruvate) and grown statically for $2-2.5$ hours at $37^{\circ} \mathrm{C}, 5 \%$ $\mathrm{CO}_{2}$ to mid-log growth phase. Expression of LEE-encoded virulence factors is known to be influenced by bacterial growth phase or enviromental factors such as culture medium, temperature and $\mathrm{pH}$ (Rosenshine et al., 1996; Abe et al., 2002; Lio and Syu, 2004). Bacteria were grown in prewarmed DMEM pH 7.4 as it has been reported that the secretion of LEE-encoded virulence factors are enhanced when EHEC strains are grown in DMEM and result in maximal adhesion to CaCo-2 cells (Abe et al., 2002; Lio and Syu, 2004). It also has been reported that environmental factors similar to those in the intestine such as $5 \% \mathrm{CO}_{2}$ enhance secretion of pathogenic proteins and VT1 production in EHEC (Yoh et al., 2003). Cells were grown to an $\mathrm{OD}_{600}$ of 0.4-0.6 (logarithmic phase) as it has been shown that early-logarithmic-phase of EPEC enhances LEE -protein secretion and increases A/E activity to HeLa epithelial cells (Rosenshine et al., 1996; Kenny et al., 1997; Vanmaele and Armstrong, 1997). $4 \mathrm{~mL}$ of the induced bacteria culture was aliquoted to $15 \mathrm{~mL}$ Falcon tubes (one for each treatment type) and centrifuged at 3,500 $\mathrm{rpm}(2,000 \mathrm{x} \mathrm{g})$ for 10 minutes at $18^{\circ} \mathrm{C}$. The resulting supernatant was removed and BS were added to the DMEM pH 7.4 at the final concentration of $2.5 \mathrm{mM}$ glycocholate (Sigma), $2.5 \mathrm{mM}$ chenocholate (Sigma), $2.5 \mathrm{mM}$ deoxycholate (Sigma), $2.5 \mathrm{mM}$ ursocholate (Sigma) and 0.15\% BS mix (Sigma) (Pope et al., 1995; Gunn, 2000). The control treatment (untreated) was resuspended in sterile filtered DMEM pH 7.4. Bacteria were incubated statically at $37^{\circ} \mathrm{C}, 5 \% \mathrm{CO}_{2}$ for 90 minutes and then cetrifugated at 3500 rpm $(2,000 \mathrm{xg})$ for 10 minutes. Supernatants were removed from all tubes and bacterial pellets were washed with 2 washes of phosphate buffered saline (PBS) (Wisent without calcium and magnesium) to ensure that all traces of BS were removed. After the second wash, the supernatant was removed and all treatments were resuspended in $4 \mathrm{ml}$ of sterile filtered DMEM $\mathrm{pH}$ 7.4. An $\mathrm{OD}_{600}$ reading was taken again to ensure that the culture was in the logarithmic phase of growth (0.4-0.6). Table 2.3 summarizes the various experimental BS treatments used in this study. 


\begin{tabular}{|l|l|}
\hline $\begin{array}{l}\text { Experimental } \\
\text { Treatment }\end{array}$ & Description \\
\hline UU & Untreated \\
Glyco & Exposed for 90 minutes with Glycocholate $(2.5 \mathrm{mM})$ \\
Cheno & Exposeded for 90 minutes with Chenodeoxycholate $(2.5 \mathrm{mM})$ \\
Deoxy & Exposed for 90 minutes with Deoxycholate $(2.5 \mathrm{mM})$ \\
Urso & Exposed for 90 minutes with Ursodeoxycholate $(2.5 \mathrm{mM})$ \\
BS & Exposed for 90 minutes with BS Mix $(0.15 \%)$ \\
Note: & BS solutions were originally made as $10 \mathrm{x}$ in $\mathrm{dH} 2 \mathrm{O}$ and diluted in DMEM pH \\
& 7.4 during the stress treatments. \\
\hline
\end{tabular}

Following BS exposure, the samples were used in viability, adhesion and cytotoxicity experiments.

\subsection{Short Chain Fatty Acid Stress of E. coli 0157:H7}

Prior to addition of the short chain fatty acids (SCFA), strains were grown in $10 \mathrm{ml}$ LB with appropriate antibiotics when necessary, in a shaking incubator at $37^{\circ} \mathrm{C}, 200 \mathrm{rpm}$ for approximately $4-5 \mathrm{~h} .2 \mathrm{ml}$ aliquots of culture were transferred to 14 Falcon tubes (one for each treatment), and centrifuged at $3500 \mathrm{rpm}(2,000 \mathrm{x} \mathrm{g})$ for 10 minutes at $18^{\circ} \mathrm{C}$. The bacterial pellet from each tube was resuspended in LB broth containing SCFAs at following final concentrations: $110 \mathrm{mM}$ sodium acetate (EMD), $70 \mathrm{mM}$ sodium propionate (Alfa Aesar), $20 \mathrm{mM}$ sodium butyrate (Alfa Aesar) and $\sim 90 \mathrm{mM}$ SCFAs mixture. SCFAs mixture is composed of $60.5 \mathrm{mM}$ acetate $(69 \%), 24.5 \mathrm{mM}$ propionate (28\%) and $3 \mathrm{mM}$ butyrate (3\%) (Lawhon et al., 2002). Following overnight growth, LB broth cultures were diluted 1:8 in DMEM pH 7.4 with appropriate SCFAs (as the same concentrations as above) and grown to mid-log growth phase. The control treatment (unshocked) was resuspended in sterile filtered DMEM pH 7.4. All bacteria were incubated statically at $37^{\circ} \mathrm{C}, 5 \% \mathrm{CO}_{2}$ for $2-2.5$ hours at, $5 \% \mathrm{CO}_{2}$ and then centrifuged at $3500 \mathrm{rpm}$ for 10 minutes. The resulting pellets were washed two times with PBS to remove any traces of SCFAs. After the second wash, the supernatant was removed and all treatments were resuspended in DMEM $\mathrm{pH}$ 7.4. $\mathrm{OD}_{600}$ readings were taken again to ensure that the cultures were in the logarithmic phase of growth (0.4-0.6) and samples were ready for use in viability, adhesion and cytotoxicity experiments. Table 2.4 
summarizes the various experimental short chain fatty acid stress treatments used in this study.

Table 2-4 Short Chain Fatty Acid Stress Experimental Treatments

\begin{tabular}{|l|l|}
\hline $\begin{array}{l}\text { Experimental } \\
\text { Treatment }\end{array}$ & Description \\
\hline UU & Unshocked \\
\hline Acetate & Shocked for $16-18 \mathrm{~h}$ with Sodium Acetate $(110 \mathrm{mM})$ \\
\hline Propionate & Shocked for $16-18 \mathrm{~h}$ with Sodium Propionate $(70 \mathrm{mM})$ \\
\hline Butyrate & Shocked for $16-18 \mathrm{~h}$ with Sodium Butyrate $(20 \mathrm{mM})$ \\
\hline Mix & Shocked for $16-18 \mathrm{~h}$ Short Chain Fatty Acid Mixture $(\sim 90 \mathrm{mM})$ \\
\hline Note: & $\begin{array}{l}\text { SCFA solutions were originally made as } 10 \mathrm{x} \text { in } \mathrm{dH} 2 \mathrm{O} \text { and diluted in } \mathrm{LB} \\
\text { broth/DMEM pH7.4 during stress treatments. } \\
\text { The colon environment was represented by a mixture containing } \sim 90 \mathrm{mM} \\
\text { composed of } 69 \% \text { acetate, } 28 \% \text { propionate and } 3 \% \text { butyrate equal to } 60.5 \mathrm{mM} \\
\text { acetate, } 24.5 \mathrm{mM} \text { propionate and } 3 \text { mM butyrate) }\end{array}$ \\
\hline
\end{tabular}

\subsection{Percent Survival of Treated E. coli $0157: \mathrm{H} 7$}

Bacteria exposed to either BS or SCFA were obtained using the appropriate Stress Treatments, as described above. A dilution series for each experimental treatment was made in PBS and the bacteria were plated out on LB agar plates, with or without appropriate antibiotic. All plates were incubated at $37^{\circ} \mathrm{C}$ for $16-18$ hours. Colony counts for plates that contained 30-300 colony forming units (CFU) were completed and used in subsequent calculations to determine the percentage of surviving organisms after BS and SCFA exposure (as assessed by culturability).

\subsection{Bacteria- Host Cell Adhesion Assay: Colony Plate Count Method}

Adhesion of BS and SCFA treated EHEC strains to either HEp-2 or CaCo-2 cells was assessed by a colony plate count assay as described previously by De Jesus et al. (2005). This method allows quantification of live organisms after stress. Using a haemocytometer (Hausser Scientific), epithelial cells grown in a $25 \mathrm{~cm}^{2}$ vented flask (BD Falcon) were enumerated and seeded in a 24-well flat bottom plates (BD Falcon) to 
approximately $80-90 \%$ confluence on the day of the experiment $\left(\approx 2 \times 10^{5}\right.$ (24-well). MEM with additives was added to each well to bring it to $0.5 \mathrm{~mL}$ (24-well) and plates were then incubated overnight at $37^{\circ} \mathrm{C}, 5 \% \mathrm{CO}_{2}$. Bacterial cultures of strains grown on LB with the appropriate antibiotics were either BS or SCFA treated using the previously described methods. Using a haemocytometer, epithelial cell counts (cells $/ \mathrm{mL}$ ) from several wells were determined and averaged. This cells $/ \mathrm{mL}$ value was used in conjunction with the bacterial $\mathrm{OD}_{600}$ value to determine the approximate amount of bacterial solution to be added to each well to achieve a multiplicity of infection (MOI) of 100:1. Results indicated that actual MOIs varied from 22-444. According to preliminary results obtained in the lab the MOI value is critical since there is a correlation between the number of bacteria loaded and adherence to epithelial cells. Results show that the more bacteria are loaded, the less they adhere to epithelial cells. To confirm the MOI, serial dilutions of all bacterial treatments were made in PBS (Wisent without calcium and magnesium) and plated out on LB agar. Only plates with 30-300 colonies were used in subsequent calculations. Prior to cell infection, the media from each well (24-well plates) was removed and cells were washed three times with PBS, $\mathrm{pH}$ 7.4. $10^{5}$ cells were infected with $10^{7}$ bacteria so the MOI of 100:1 was achieved and fresh DMEM without antibiotics was added to each well to bring the volume up to either $0.5 \mathrm{~mL}$ ( 24 well plate) or $3 \mathrm{~mL}$ ( 6 well plate). The infected monolayers were incubated at $37^{\circ} \mathrm{C}, 5 \% \mathrm{CO}_{2}$ for 4 hours of infection. After a total of $4 \mathrm{~h}$ incubation, the media and non-adherent bacteria were removed, and the wells washed five times with PBS pH 7.4. Adherent bacteria and remaining epithelial cells were detached by adding $250 \mathrm{uL}$ trypsin-EDTA ( $0.25 \%$ trypsin; $1 \mathrm{mM}$ EDTA.4Na). After approximately two minutes incubation at $37^{\circ} \mathrm{C}, 5 \% \mathrm{CO}_{2}, 50 \mathrm{uL}$ of FBS was used to stop the trypsin reaction, and the cells from each well were added to $1.5 \mathrm{~mL}$ microcentrifuge tubes. The wells were rinsed with $200 \mathrm{uL}$ of cold sterile distilled deionized $\mathrm{H}_{2} \mathrm{O}\left(\mathrm{ddH}_{2} \mathrm{O}\right)$ and this was added to the tubes. Complete detachment of cells from wells was verified microscopically. Epithelial cells were lysed with $500 \mathrm{ul}$ cold sterile $\mathrm{ddH}_{2} \mathrm{O}$ and then were added to each tube (total $1 \mathrm{~mL}$ per well, for 24 well plates). The number of viable bacteria bound to adherent cells, were quantified by serial dilution in PBS performed for each treatment (with triplicates). The appropriate dilutions $\left(10^{-5}\right.$, $10^{-6}, 10^{-7}$ ) were plated on LB agar containing antibiotics when necessary, and incubated 
between $16-18$ hours at $37^{\circ} \mathrm{C}$. The following day, the colonies were counted and plates that had between 30-300 colonies were used for further calculations and statistical analysis.

\subsection{Impact of Erythromycin-Mediated Inhibition of Protein Synthesis on Bacteria-Host Adhesion}

A modified version of a previously described method by de Jesus et al. (2005) was used to assess the involvement of newly synthesized adhesins after stress. E. coli 86-24 cultures were grown on LB followed by either BS or SCFA treatment. Following the stress treatment, $4 \mathrm{~mL}$ of neutralized bacterial suspension, in DMEM pH 7.4 for each treatment, was divided into two tubes of $2 \mathrm{~mL}$ aliquots; $2 \mathrm{~mL}$ of each treatment will be subjected to erythromycin treatment and the other without. All tubes were centrifuged at $3,300 \mathrm{rpm}(2,000 \mathrm{xg})$ for 10 minutes at $18^{\circ} \mathrm{C}$ and resuspended in $2 \mathrm{~mL}$ DMEM pH 7.4 with or without $0.06 \mathrm{mg} / \mathrm{mL}$ erythromycin (Sigma), depending on the treatment. All tubes were left at room temperature for 30 minutes prior to cell infection. The effect of erythromycin on BS stressed bacterial adhesion to HEp- 2 cells was determined by a 24 well colony plate count assay as previously described in Section 2.6.

\subsection{Bile Salt/Short Chain Fatty Acids Treated E. coli 0157:H7 86-24 Growth during Infection Time}

This experiment was performed to assess the impact of BS and SCFA exposure on the growth of E. coli 86-24 throughout the infection time in a four hour bacterial-host cell adhesion assay. Cultures of E. coli 86-24 were grown in LB, then either BS or SCFA stressed using the previously described BS or SCFA treatment in Section 2.3, Section 2.4. After stress, the treatments were normalized using prior viability data to ensure that the initial inoculum was approximately the same. To validate the normalization, a dilution series was prepared in PBS for each normalized treatment and plated on LB agar and incubated at $37^{\circ} \mathrm{C}$ for $16-18$ hours. $100 \mathrm{uL}$ of normalized bacteria from each treatment was added to an empty (cell-free) well from a 24-well plate and MEM (without additives) was added to each well to bring the volume to $0.5 \mathrm{~mL}$, and incubated at $37^{\circ} \mathrm{C}, 5 \% \mathrm{CO}_{2}$. 
After $4 \mathrm{~h}$ incubation time, the media and bacteria were removed and added to $1.5 \mathrm{~mL}$ microcentrifuge tubes. To determine the impact of BS and SCFA stress on bacterial growth, serial dilutions using PBS were performed for each treatment. The appropriate dilutions $\left(10^{-5}, 10^{-6}, 10^{-7}\right)$ were plated on LB agar (triplicates) and incubated between 1618 hours at $37^{\circ} \mathrm{C}$. Following incubation, colonies were counted and plates that had between 30-300 colonies were used in subsequent calculations and statistical analysis.

\subsection{Qualitative Microscopic HEp-2 cell Adhesion Assay after Exposure to Bile Salts/Short Chain Fatty Acids}

One day prior to experiment, epithelial cells grown in a $25 \mathrm{~cm} 2$ vented culture flask (BD Falcon) were enumerated with the aid of a haemocytometer (Hausser Scientific) and then added to 6-well flat bottom plates with coverslips (BD Falcon) so that the cell monolayer would be approximately $80-90 \%$ confluent on the experimental day; 8 x 105 cells/well in a 6-well plate. DMEM with additives was then added to each well to bring the volume to $3 \mathrm{~mL}$. The plates were then incubated overnight at $37^{\circ} \mathrm{C}, 5 \% \mathrm{CO}$. LB culture of E. coli 86-24 was subjected to either BS or SCFA stress using previously described BS or SCFA Treatment (Section 2.3 and 2.4). Epithelial cell counts (cells $/ \mathrm{mL}$ ) from 2-3 wells and the approximate amount of bacterial solution to be added to each well were determined as previously described in Bacteria- Host Cell Adhesion Assay: Colony Plate Count Method (Section 2.6). DMEM (without additives) was added to each well to bring the volume up to $3 \mathrm{~mL}$ and the well plates were incubated at $37^{\circ} \mathrm{C}, 5 \% \mathrm{CO} 2$ for four hours. After 4-hour infection time, media and non adherent cells were removed and wells with coverslips were washed five times with PBS. The cells were fixed by adding 2 $\mathrm{ml}$ of $100 \%$ methanol (Sigma) and incubated at room temperature for 5 minutes. After incubation, the cells were stained by adding $2 \mathrm{ml}$ of Giemsa stain (Sigma) and incubated at room temperature for 45 minutes. Cells were washed few times with $\mathrm{dH} 2 \mathrm{O}$. For microscopic preparation, the coverslip from each well was carefully removed with forceps and rinsed with acetic acid solution (1\%), placed on a glass slide (25 $\mathrm{mm} \times 75$ $\mathrm{mm}, \mathrm{VWR})$. Coverslips were then sealed on the glass and were ready for examination 
under the light microscope. A Leica DM 5000B Microscope equipped with camera was used. Images were taken at $630 \mathrm{x}$ magnification using immersion oil.

\subsection{Vero Cell Cytotoxicity Assays}

Shiga toxin produced during stress of $E$. coli $0157: \mathrm{H} 7$ 86-24 was assessed by exposing Vero cells in microtiter cultures to dilutions of toxin. A modified version of The Quantitative Microtiter cytotoxicity assay as previously descried by Karmali et al., (1985) and Khine et al., (2004) was used to assess Shiga toxin produced during stress. Briefly, one day before the experiment, Vero cells grown to confluency $\left(80-90 \%, 75 \mathrm{~cm}^{2}\right.$ flasks) were trypsinized with $2 \mathrm{~mL}$ of trypsin-EDTA and incubated for approximately 2 minutes at $37^{\circ} \mathrm{C}, 5 \% \mathrm{CO}_{2}$. Following trypsinization, cells were counted and suspended to the desired concentration in growth medium. Seven 96-well microtiter plates (BD Falcon), one for each experimental treatment type (BS or SCFA) and one for the control were seeded with $200 \mathrm{uL}$ of the vero cell solution using a multichannel pipettor. Monolayers were established by incubating the plates overnight at $37^{\circ} \mathrm{C}, 5 \% \mathrm{CO}_{2}$. Negative controls were wells containing cells not exposed to toxin that were included on each plate. LB culture of 86-24 was subjected to either BS or SCFA stress as previously described in Section 2.3 and 2.4. After stress, the treatments were normalized using prior viability data to ensure that the initial innoculum was approximately the same. To validate the normalization, a dilution series was prepared in PBS for each normalized treatment and plated on LB agar and incubated at $37^{\circ} \mathrm{C}$ for $16-18$ hours. For verotoxin production, $1 \mathrm{~mL}$ of each bacterial treatment was added to $14 \mathrm{~mL}$ of Penassay broth (antibiotic medium no. 3; Difco Laboratories) and incubated overnight at $37^{\circ} \mathrm{C}$ and 200 rpm. Following overnight incubation, $2 \mathrm{~mL}$ of each treatment were used to obtain $\mathrm{OD}_{600}$ readings and/or serial dilutions were performed in PBS. The appropriate serial dilution were plated on $\mathrm{LB}$ agar and incubated at $37^{\circ} \mathrm{C}$ for $16-18$ hours. After all treatments, cells were centrifuged at $3,500 \mathrm{rpm}(2,000 \times \mathrm{g})$ for 10 minutes at $18^{\circ} \mathrm{C}, 10 \mathrm{~mL}$ of the supernatant was filter sterilized using $0.2 \mathrm{um}$ membrane filters and $10 \mathrm{cc}$ Leur-Lok syringes (BD Falcon). The supernatant filtrates were stored at $-20^{\circ} \mathrm{C}$ for no longer than a week until use. The remaining supernatant was aspirated and bacterial pellet was 
resuspended in $1.5 \mathrm{~mL}$ of $0.1 \mathrm{mg} / \mathrm{mL}$ colymycin (Parker Davis) and incubated statically at $37^{\circ} \mathrm{C}$ for 30 minutes. The treatments were then centrifuged at $3300 \mathrm{rpm}$ for 10 minutes at $18^{\circ} \mathrm{C}$ and the periplasmic extracts were filter sterilized using 0.2 um membrane filters and $3 \mathrm{cc}$ leur-lok syringes (BD Falcon). The resulting periplasmic extracts were stored at $-20^{\circ} \mathrm{C}$ for no longer than a week until use. On the day of the experiment, frozen experimental extracts and filtrates were thawed and dilutions of the samples $1 / 1,1 / 5$, $1 / 25,1 / 125,1 / 625$ and 1/15625 were made in MEM (no additives) and $50 \mathrm{uL}$ of each dilution for each experimental treatment (both periplasmic extracts and supernatant filtrates) was added, with replicates, to the wells. For the control wells, $50 \mathrm{uL}$ of MEM (no additives) were added. All plates were incubated for $2-3$ days at $37^{\circ} \mathrm{C}, 5 \% \mathrm{CO}_{2}$. After incubation, the detached cells, medium and toxin in the wells were removed by vigorous shaking. Remaining live cells in each well were fixed with $65 \mathrm{uL}$ of a $2 \%$ solution of formalin $(0.05 \%$ of a $40 \%$ formaldehyde $(\mathrm{v} / \mathrm{v}), 95 \%$ PBS $(\mathrm{v} / \mathrm{v}))$. The plates were incubated at room temperature for approximately 1-2 minutes and fixative was removed from the wells. The wells of the plates were stained with $65 \mathrm{uL}$ of Crystal Violet Tissue Culture stain $(0.26 \%$ of $0.5 \%$ Crystal Violet Stock Solution (v/v), $0.05 \%$ absolute ethanol (v/v), $0.05 \%$ of $40 \%$ formaldehyde (v/v), $0.64 \%$ of PBS (v/v)) and incubated at room temperature for a minimum of 20 minutes. The excess crystal violet stain was removed and the wells were rinsed four times with $\mathrm{ddH}_{2} \mathrm{O}$ and the plates were left to air dry. To quantify the results, $100 \mathrm{uL}$ of $10 \%$ acetic acid solution (Fisher Scientific) was added to solubilize the Crystal Violet and the absorbance was determined at $570 \mathrm{~nm}$ in an ELISA plate reader.

\subsection{Statistical Analysis}

The mean and standard deviations of all quantitative adhesion and cytotoxicity assay results were calculated. A two-tailed Student $t$-test was used to determine if the results for E. coli $\mathrm{O} 157: \mathrm{H7}$ after exposure to BS or SCFA were significantly different from the control unstressed cells. $P$-values less than or equal to 0.05 were considered statistically significant. 


\section{Results}

\subsection{Bile Salt Treatment Enhances $E$. coli 0157:H7 Survival}

The viability of $E$. coli $\mathrm{O} 157: \mathrm{H} 7$ was predicted to be affected after BS exposure. To determine whether E. coli $\mathrm{O} 157: \mathrm{H} 7$ strains were capable of survival after BS treatment, the viability of wild-type strain 86-24 was assessed before and after BS as described in the Methods Section 2.6 using a plate count method. The viability of BS stressed EHEC 86-24 wt was increased $1.2-1.5$ fold relative to that of unshocked (Fig 3.1). BS treatment resulted in significantly increased viability of $\log$ phase E. coli $\mathrm{O} 157: \mathrm{H} 7$ strains relative to that of untreated. Similar trends were obtained with other E. coli O157:H7 strains such as $8624 e a e \Delta 10$ and $8624 e a e \Delta(\mathrm{pEB} 310)$ which showed significantly increased survival levels compared to untreated bacteria (data not shown).

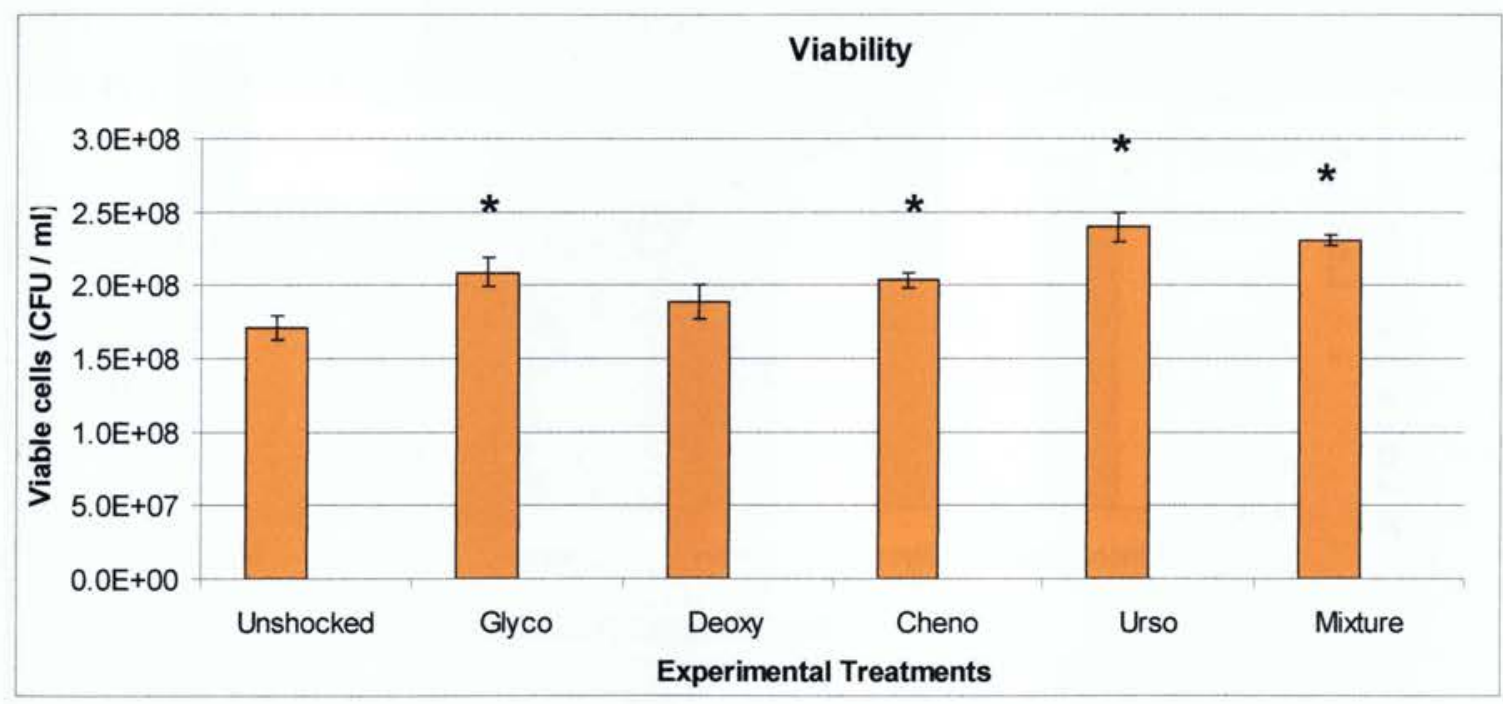

Figure 3.1-1 BS Treatment Enhances EHEC 0157:H7 Survival

Effect of BS exposure on E. coli O157:H7 86-24 viability was assessed by plate count assay (n=3). Values are means \pm standard deviations and are representative of a minimum 4 experiments. BS Stress Treatment: $90 \mathrm{~min}, 37^{\circ} \mathrm{C}, 5 \% \mathrm{CO}_{2}$ Cheno/Deoxy/Urso/Glyco: $2.5 \mathrm{mM}$; BS Mixture: $0.15 \% \mathrm{w} / \mathrm{v}$. [Untreated: Control (Untreated for 90 minutes); Glyco: Glycocholate treated for 90 minutes; Deoxy: Deoxycholate treated for 90 minutes; Cheno: Chenodeoxycholate treated for 90 minutes;;); Urso: Ursodeoxycholate treated for 90 minutes; Mixture: BS Mixture treated for 90 minutes]. ${ }^{*}$ Significantly different from corresponding control $(\mathrm{P}<0.05)$. 


\subsection{Bile Salt Treatment Enhances E.coli 0157:H7 Adhesion to Human Epithelial Cells}

To judge the level of adherence of E. coli $0157: \mathrm{H} 7$ strains to human epithelial cells, adhesion assays before and after BS stress were conducted. Bacterial adhesion was determined by plate count assay (Fig 3.2-1) and light microscopy was used as as a complimentary method (Figure 3.2-2). Adhesion of BS mixture and glycholate treated EHEC 86-24 to epithelial cells was significantly higher than that of the untreated control EHEC 86-24. Adhesion enhancement factors ranged from 1.5 - 2.1 for HEp-2 cells to 4.8 for Caco-2 cells (Fig 3.2-1, Figure 3.3-1 and Table 1 Supplementary Data).

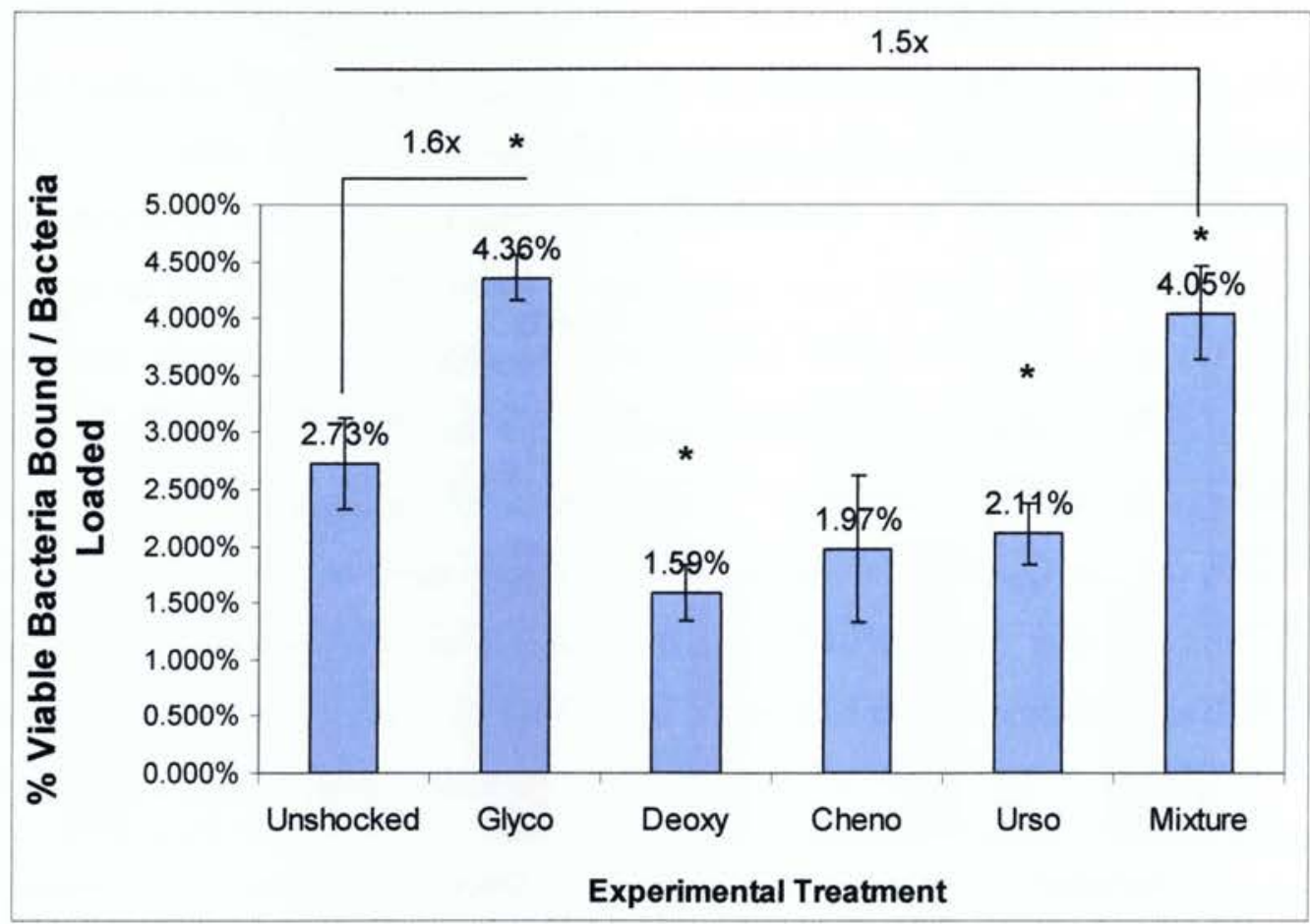

Figure 3.2-1 BS Treated $E$. coli O157:H7 86-24 Show Enhanced Adhesion to HEp-2 Cells

Effect of BS treatment of E. coli O157:H7 86-24 on adhesion to HEp-2 cells (MOI range 45-57) was assessed by plate count assay ( $n=4-6$ for each experiment). The results of 2 independent experiments are shown and the values are means \pm standard deviations. BS Stress Treatment: $90 \mathrm{~min}, 37^{\circ} \mathrm{C}, 5 \% \mathrm{CO}_{2}$ Cheno/Deoxy/Urso/Glyco: 2.5mM; BS Mix: $0.15 \%$ w/v. [Untreated: Control (Untreated for 90 minutes); Glyco: Glycocholate treated for 90 minutes; Deoxy: Deoxycholate treated for 90 minutes; Cheno: Chenodeoxycholate treated for 90 minutes;; Urso: Ursodeoxycholate treated for 90 minutes; Mixture: BS Mixture treated for 90 minutes]. *Significantly different from corresponding control $(\mathrm{P}<0.05)$; 
In agreement with the previous results obtained, light microscopy images of infected Giemsa stained cells showed obvious increased adherence of BS mixture and glycocholate treated E. coli $\mathrm{O} 157: \mathrm{H} 7$ as compared with untreated strains (Figure 3.2.2). HEp-2 cell monolayers were incubated with BS treated EHEC 86-24 for 4 hours as described in Materials \& Methods (Section 2.6). Adhesion patterns were typical of those reported for EHEC adherence to cultured epithelial cells (Torres and Kaper, 2003).

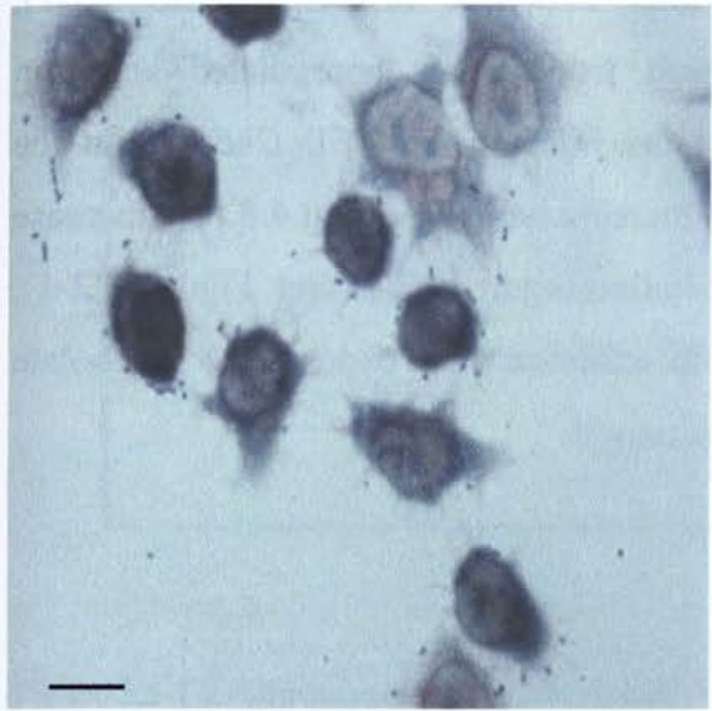

A

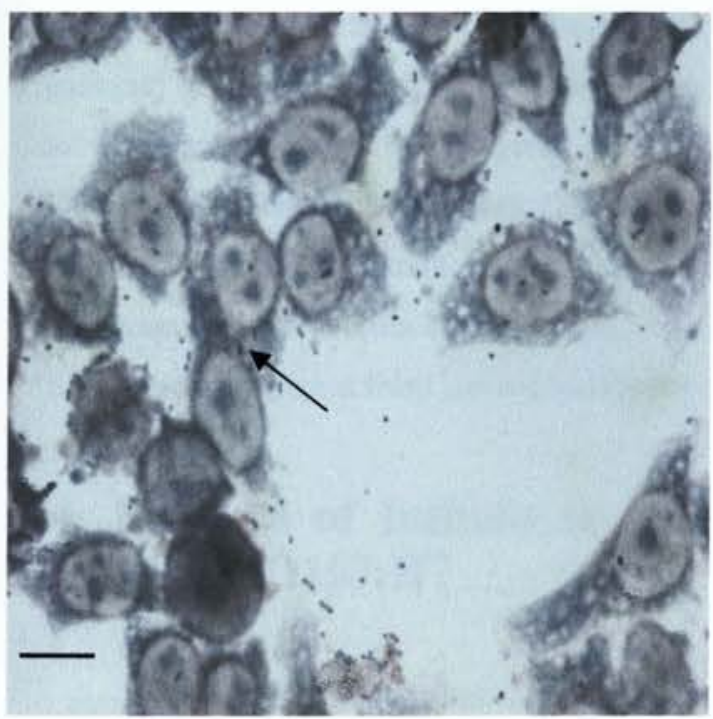

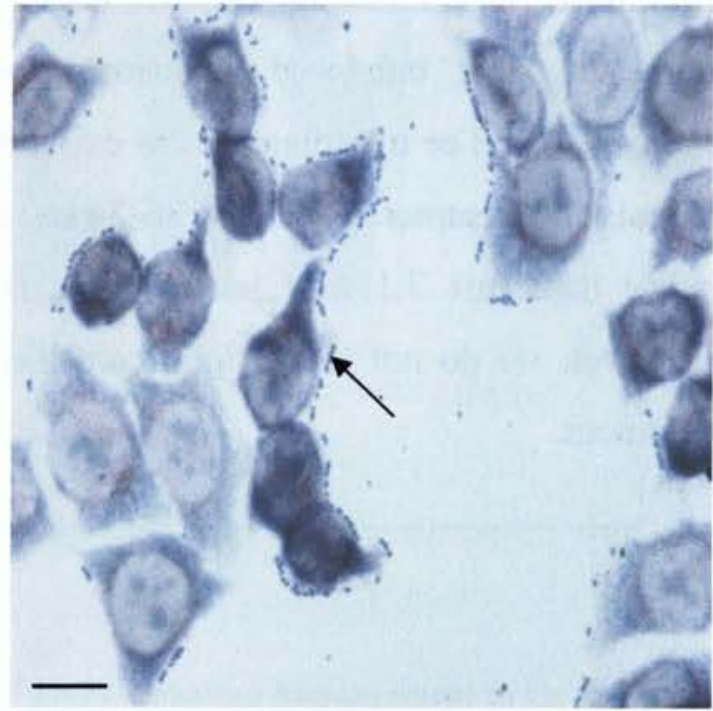

B

C

Figure 3.2-2 Qualitative Comparison of BS Treated $E$. coli O157:H7 86-24 Adhesion to HEp-2 Cells

Effect of BS Treatment of E. coli O157:H7 86-24 on adhesion to HEp-2 cells using light microscopy. A: Untreated control; B: BS Mixture Treatment; C: Glycocholate Treatment; BS Treatment: $90 \mathrm{~min}, 37^{\circ} \mathrm{C}$, $5 \%$ CO2 Glyco: $2.5 \mathrm{mM}$; BS Mixture: $0.15 \% \mathrm{w} / \mathrm{v}$. The black arrows indicate either BS or Glycocholate treated $E$ coli cells attached to HEp- 2 cells. Scale bar on A,B, and $\mathrm{C}$ represents 7 microns 


\subsection{Erythromycin Treatment Abrogates EHEC 0157:H7 Adhesion Enhancement}

To investigate further whether the adhesion enhancement is a result of increased expression of adhesins, erythromycin, below the MIC, was used to inhibit protein synthesis (de Jesus et al., 2005). Pretreatment of BS treated bacteria with erythromycin eliminated the adhesion enhancement seen after exposure to BS (Figure 3.3-1), suggesting that enhanced adhesion could result from either upregulated adhesion expression and or modifications to existing adhesins. Interestingly, with $\mathrm{Caco} 2$ cells, the BS mixture treatment of EHEC 86-24 caused an increase which is about 4.8 fold increase rather than just 2.1 fold increase for HEp-2 cells (Figure 3.3-1 and Figure 3.2-1). However, we do not see a significant increase in adhesion after exposure to glycholate treatment. 


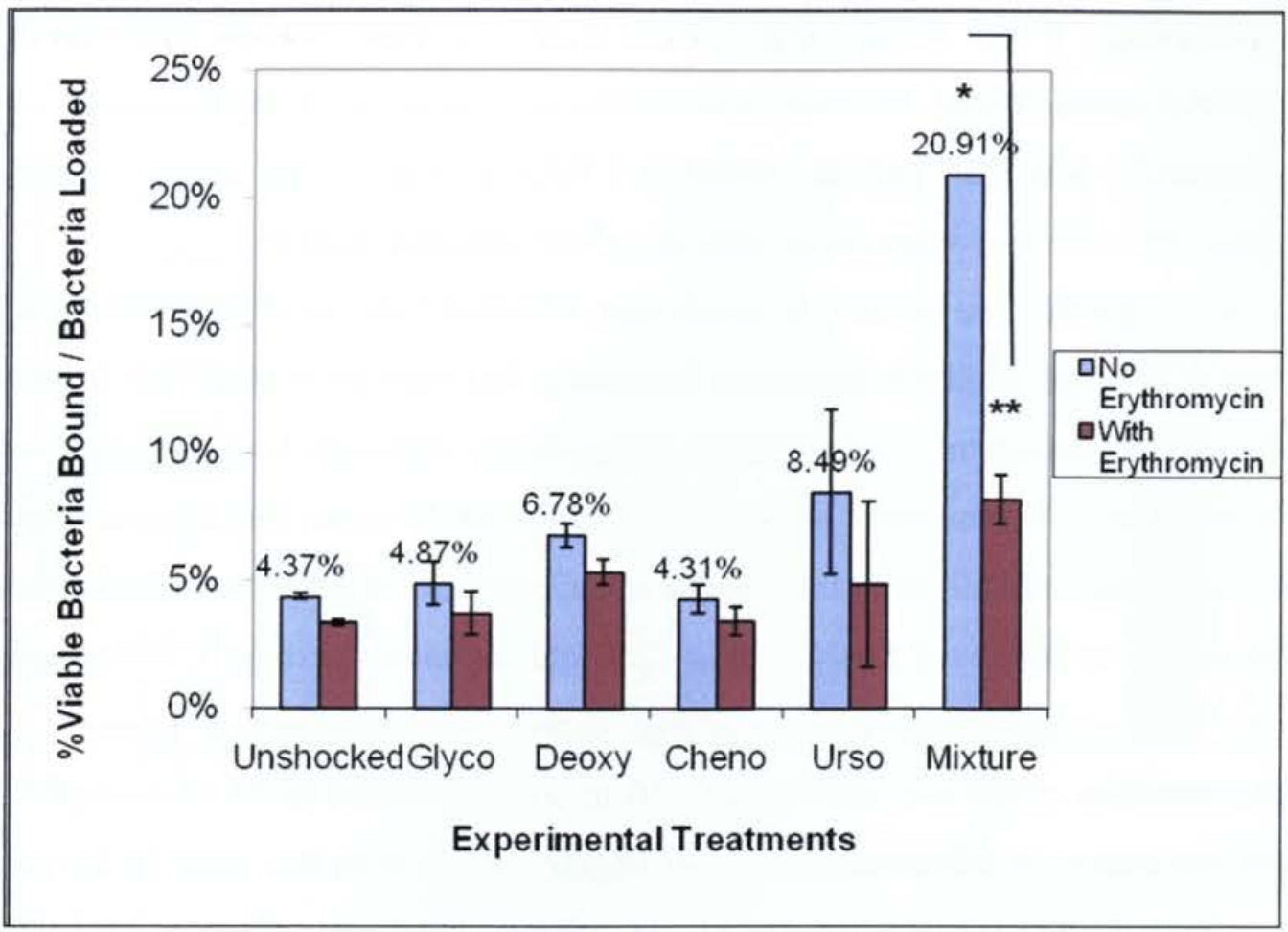

Figure 3.3-1 Erythromycin Abrogates E. coli O157:H7 86-24 Adhesion Enhancement to Caco-2 Cells. The effect of erythromycin on BS treatment of E. coli O157:H7 86-24 adhesion to Caco-2 cells (MOI range 114-150) was assessed by plate count assay $(n=6$, ) and the values are means \pm standard deviations. BS Treatment: $90 \mathrm{~min}, 37^{\circ} \mathrm{C}, 5 \% \mathrm{CO}_{2}$ Cheno/Deoxy/Urso/Glyco: $2.5 \mathrm{mM}$; BS Mix: $0.15 \% \mathrm{w} / \mathrm{v}$. [Untreated: Control (Untreated for 90 minutes); Cheno: Chenodeoxycholate treated for 90 minutes; Deoxy: Deoxycholate treated for 90 minutes; Glyco: Glycocholate treated for 90 minutes); Urso: Ursodeoxycholate treated for 90 minutes; Mixture: BS Mixture treated for 90 minutes].

*Significantly different from corresponding unshocked control $(\mathrm{P}<0.05)$

**Significantly different from corresponding BS treatment $(\mathrm{P}<0.05)$

\subsection{No Role of Intimin in Enhanced Adhesion of Bile Salt Treated EHEC 0157:H7}

To explore the contribution of intimin, a known adhesin in increased adhesion after BS stress, intimin mutants (86-24eae $\Delta 10$ and 86-24eae $\Delta(\mathrm{pEB} 310))$ were used to investigate adherence to human intestinal cells with and without BS exposure. To achieve this aim, first we ran a control experiment to compare adhesion capability of $E$. coli $\mathrm{O} 157: \mathrm{H} 7$ wild-type 86-24 and intimin mutants obtained with the tissue culture 
adherence assays. It was verified that intimin negative mutant adhered significantly less than parental strain ( 8 fold decrease) and intimin recomplemented mutant strain adhered only marginally less than parental strain (1.4 fold decrease). This result confirms the importance of intimin in adhesion to cells in culture (data not shown).

Although the eae mutant is much less adherent than wild-type, adhesion after exposure to BS was similarly increased, indicating that intimin is likely not involved in the adhesion enhancement (Figure 3.4-1). Interestingly, although both untreated and BS treated 86-24eae $\Delta 10$ were only weakly adherent (about 10 times less than untreated wt $86-24)$ there was a significantly increase in adhesion of BS mixture treated 86-24eae $\Delta 10$ relative to that of untreated $86-24 e a e \Delta 10$ (2.3 fold adhesion increase ), which suggests that the BS mixture may be triggering expression of adhesins (Figure 3.4-1). Complementation of the eae gene in pEB310 in 86-24 (resulted in 86-24eae $\Delta(\mathrm{pEB} 310)$ ) showed restoration of adherence to a level slightly higher than that seen in the parental strain (1.5 fold adhesion increase after BS mixture treatment) (Figure 3.4-2). Taken together, the adherence data suggest that intimin is not the adhesin responsible for the increase in adhesion observed after BS treatment.Other colonization factors may contribute to increased adhesion to human intestinal cells. 


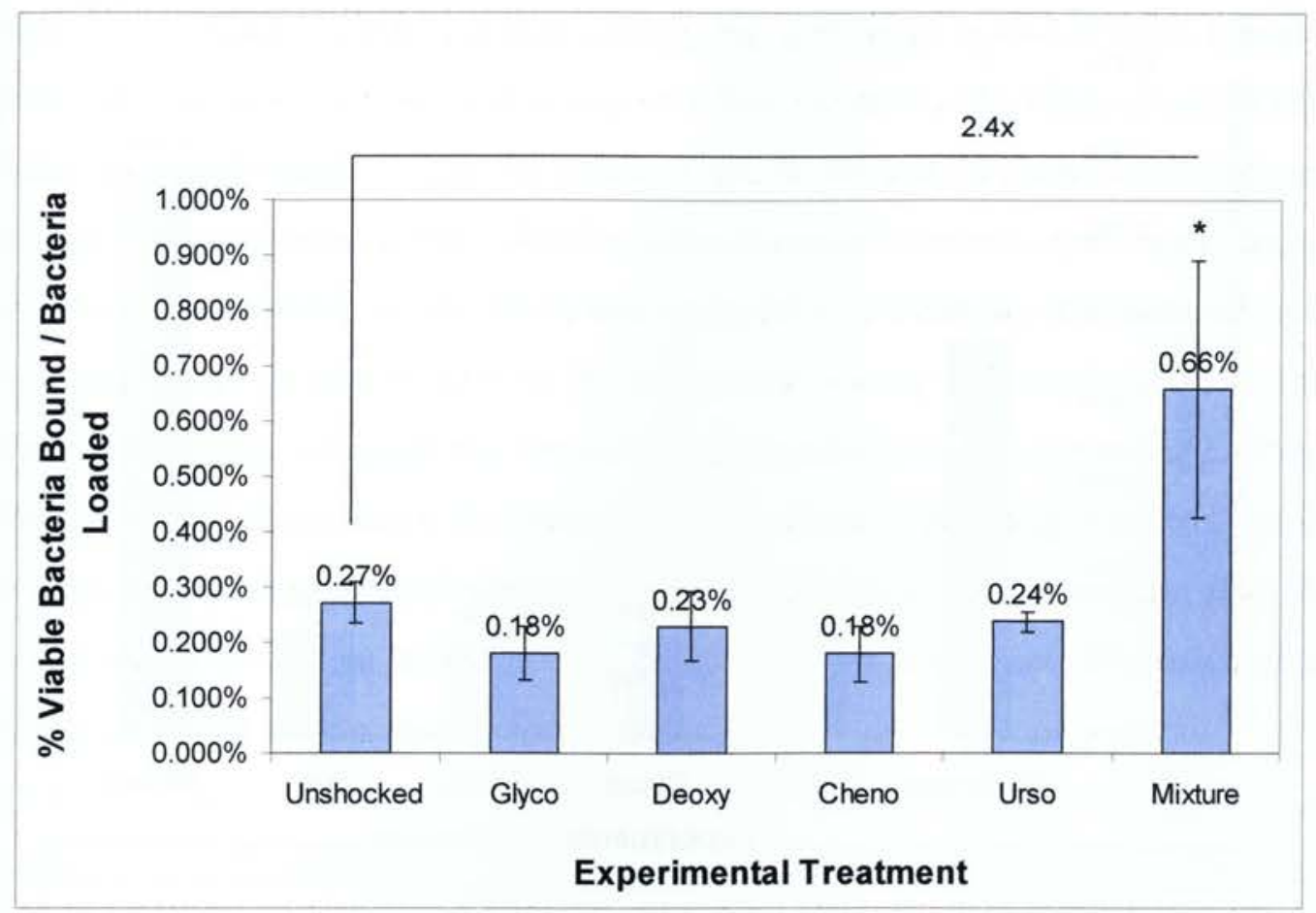

Figure 3.4-1 BS Treated $E$. coli 0157:H7 86-24 eae $\Delta 10$ Show Enhanced Adhesion to HEp-2 Cells Effect of BS shock of E. coli O157:H7 86-24 eae 10 on adhesion to HEp2 cells (MOI range 57-105) was assessed by plate count assay $(n=3)$. Data shown are representative of 2 independent experiments and the values are means \pm standard deviations. BS Treatment: $90 \mathrm{~min}, 37^{\circ} \mathrm{C}, 5 \% \mathrm{CO}_{2} \mathrm{Cheno} / \mathrm{Deoxy} / \mathrm{Urso} / \mathrm{Glyco}$ : $2.5 \mathrm{mM}$; BS Mixture: $0.15 \%$ w/v. [Untreated: Control (Untreated for 90 minutes); Glyco: Glycocholate treated for 90 minutes; Deoxy: Deoxycholate treated for 90 minutes; Cheno: Chenodeoxycholate treated for 90 minutes; ; Urso: Ursodeoxycholate treated for 90 minutes; Mixture: BS Mixture treated for 90 minutes]. *Significantly different from corresponding control $(\mathrm{P}<0.05)$; 


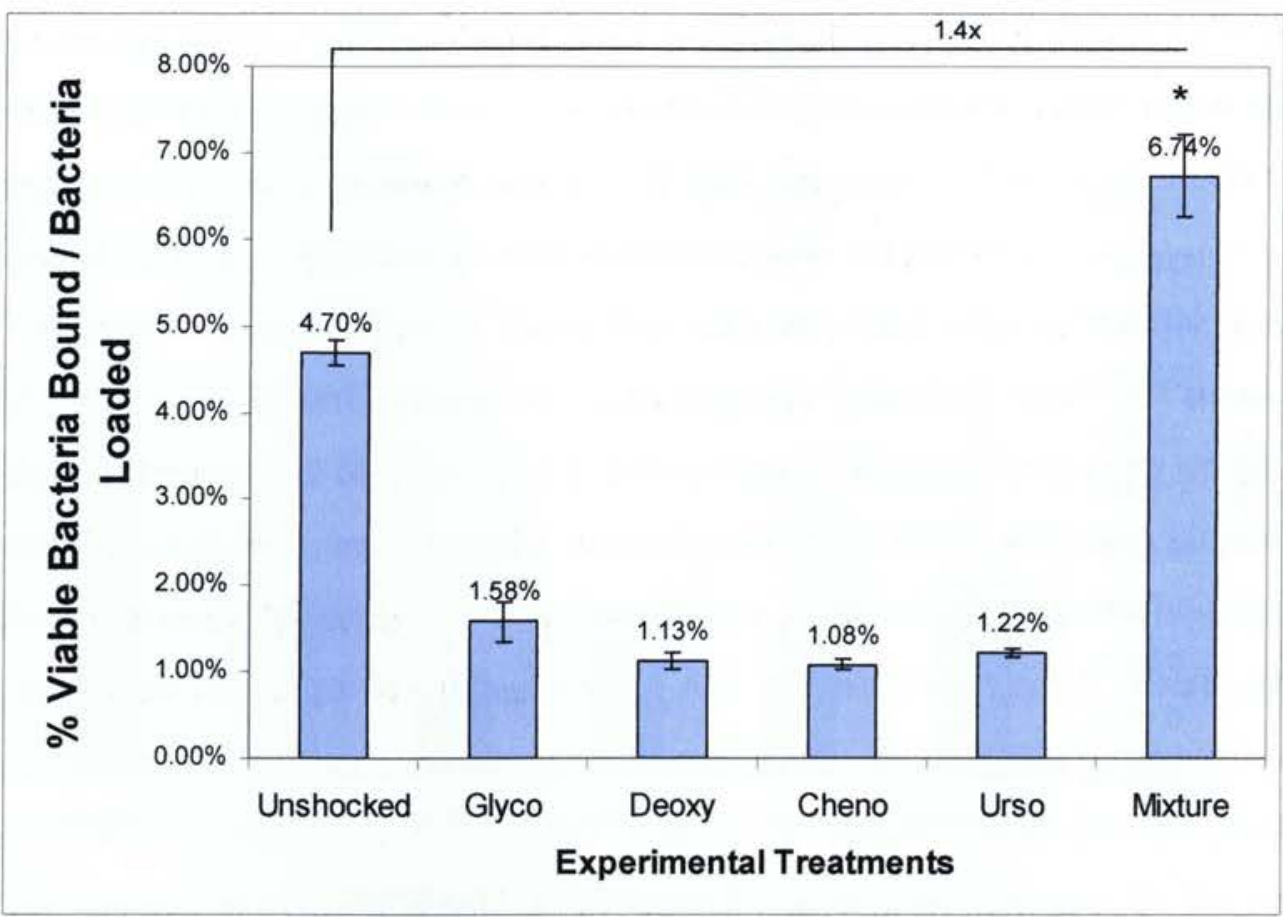

Figure 3.4-2 BS Treated E. coli O157:H7 8624 eae $\Delta$ (pEB310) Show Enhanced Adhesion to HEp-2 Cells.

Effect of BS shock of $E$. coli O157:H7 86-24 eae $\Delta 10$ (pEB310) on adhesion to HEp-2 cells (MOI range 6792) was assessed by plate count assay $(n=3)$. Data shown are representative of 2 independent experiments and the values are means \pm standard deviations. BS Treatment: $90 \mathrm{~min}, 37^{\circ} \mathrm{C}, 5 \% \mathrm{CO} 2$ Cheno/Deoxy/Urso/Glyco: $2.5 \mathrm{mM}$; BS Mixture: $0.15 \% \mathrm{w} / \mathrm{v}$. [Untreated: Control (Untreated for 90 minutes); Glyco: Glycocholate treated for 90 minutes; Deoxy: Deoxycholate treated for 90 minutes; Cheno: Chenodeoxycholate treated for 90 minutes; Urso: Ursodeoxycholate treated for 90 minutes; Mixture: BS Mixture treated for 90 minutes].

*Significantly different from corresponding control $(\mathrm{P}<0.05)$;

\subsection{No Role of Iha in Enhanced Adhesion of Bile Salt Treated EHEC O157:H7}

To investigate whether Iha (bacterial adherence-conferring protein) is a prerequisite for increased adhesion in BS treated EHEC, iha mutants and nonpathogenic E. coli complemented with iha were used in quantitative adherence assays. Firstly, 86$24 \Delta$ iha mutant adhered at about $70 \%$ of the wt strain $86-24$ level $(1.69 \%$ and $2.5 \%$ 
respectively) (Figure 3.5-1). These results are consistent with previous reports that indicate that iha plays a role in adhesion of EHEC to host cells (Tarr et al., 2000). To

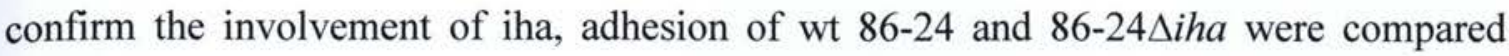
before and after exposure to BS. Similar adhesion enhancements were noted for strains

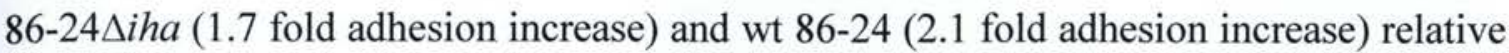

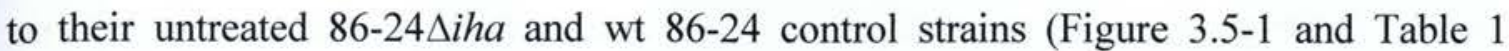
Suplementary Data). Although the iha mutant is significantly less adherent $(2.88 \%)$ than wt $86-24(5.29 \%)$, there was a similarly increased adhesion after exposure to BS mixture relative to that of untreated controls (Figure 3.5-1). These results suggest that E. coli O157:H7 86-24 does not utilize iha for the increased adherence observed after BS exposure, and other factors must be involved.

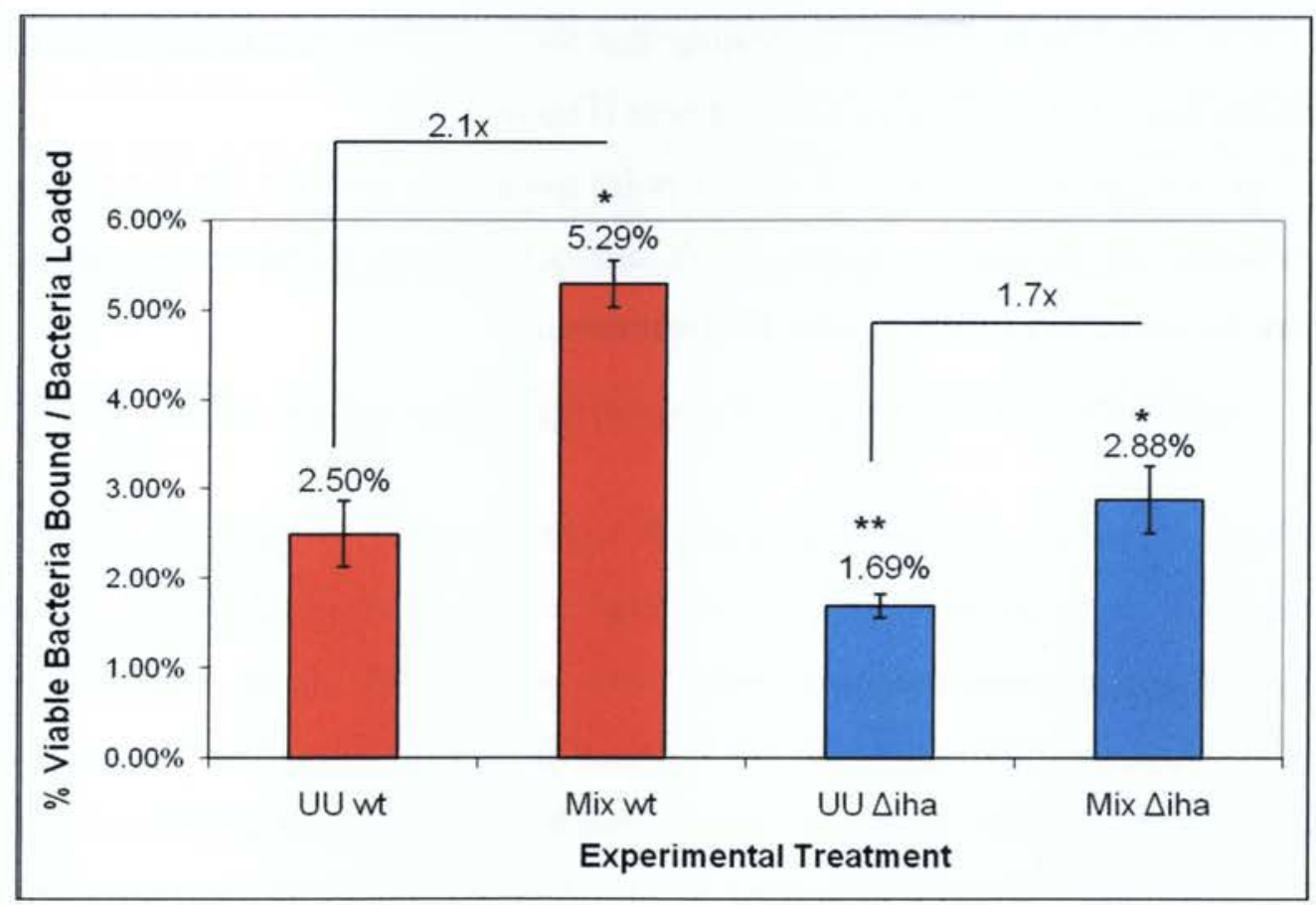

Figure 3.5-1 BS Treated E. coli O157:H7 86-24 $\Delta$ iha Show Enhanced Adhesion to HEp-2 Cells

Effect of BS shock of $E$. coli O157:H7 8624Aiha on adhesion to HEp-2 cells (MOI range 99-101) was assessed by plate count assay $(n=3)$. Results shown are representative of 2 independent experiments. Values are means \pm standard deviations. BS Treatment: $90 \mathrm{~min}, 37^{\circ} \mathrm{C}, 5 \% \mathrm{CO}_{2}$ BS Mix: $0.15 \% \mathrm{w} / \mathrm{v}$. [Untreated: Control (Untreated for 90 minutes); Mixture: BS Mixture treated for 90 minutes].

*Significantly different from corresponding control $(\mathrm{P}<0.05)$

${ }^{* *}$ Significantly different from corresponding untreated $(\mathrm{P}<0.05)$; 
Since Iha has been described to contribute to enhanced adherence of non-adherent laboratory E. coli strains, we tested BS treated ORN172(pIHA) and ORN172(pSK+) to determine if tha is responsible for the enhanced adhesion after BS exposure. E. coli ORN172(pIHA) is a nonvirulent laboratory strain which expresses the iha in trans, while ORN172(pSK + ) carries the plasmid alone and and does not carry iha in its chromosome (Tarr et al., 2000). Untreated E. coli ORN 172(plha) adhered 4x more than ORN172 $(\mathrm{pSK}+)$ demonstrating that Tha plays a major role in adhesion when transgenically expressed in a non-pathogenic E. coli background (Figure 3.5-2). There is no significant increase in adhesion of mixture treated ORN 172(pIHA) relative to the untreated, confirming that Iha does not likely play a role in BS induced adhesion of $E$. coli $\mathrm{O} 157: \mathrm{H} 7$ (Figure 3.5-2). There is also no significant increase in the mixture treated in ORN172 $(\mathrm{pSK}+)$ relative to the untreated, suggesting that there is no upregulation of adhesins in the nonpathogenic ORN 172 after BS treatment (Figure 3.5-2).

Taken together both sets of results point toward no role for iha suggesting that other adhesins or virulence factors which are not present in laboratory strains are necessary for increased adhesion after BS treatment. 


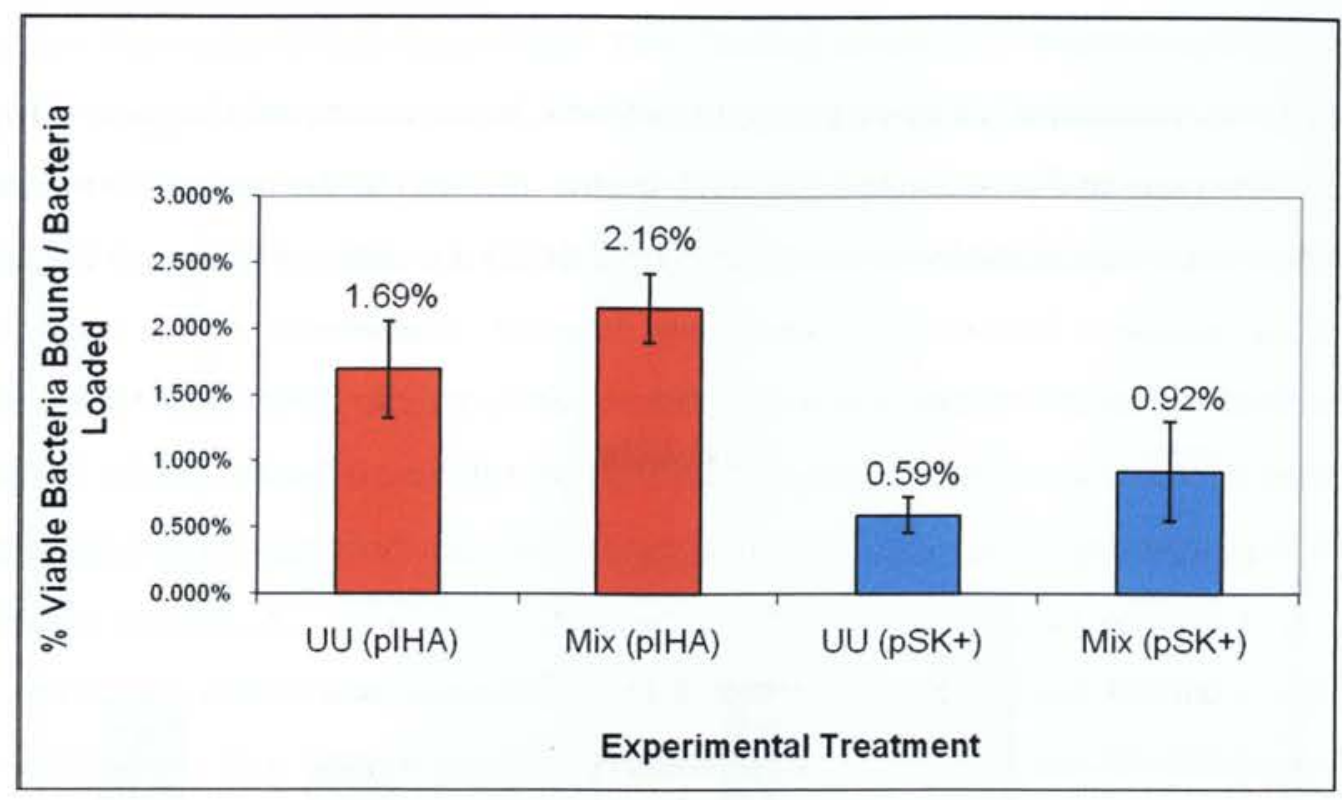

Figure 3.5-2 BS Treated E.coli ORN 172(pIHA) and (pSK+) Adhesion to HEp-2 Cells

Effect of BS shock of E.coli ORN172(pIHA) and ORN172(pSK + ) on adhesion to HEp-2 cells was assessed by plate count assay $(n=3)$. Values are means \pm standard deviations. BS Stress Treatment: $90 \mathrm{~min}, 37^{\circ} \mathrm{C}$, $5 \%$ CO2; BS Mixture: $0.15 \%$ w/v. [Untreated: Control (Untreated for 90 minutes); Mixture: BS Mixture treated for 90 minutes]. MOI range for pIHA 182-199 and MOI range for pSK+424-444

\subsection{Short Chain Fatty Acids Decrease E. coli O157:H7 Viability}

Several species of Gram-negative bacteria are known for their considerable tolerance to SCFA (Lasko et al., 2000; Arnold et al., 2001; Lawhon et al., 2002; Polen et al., 2003; Maurer et al., 2005). Less toxic effect on some microorganisms has been attributed to the weak acid nature of most of the organic acids. However, SCFA are capable of exhibiting bacteriostatic and bactericidal properties. Most of these compounds can easily penetrate the lipid membrane of the bacterial cell where they dissociate into anions and protons and may stress bacteria by depleting cellular energy (Ricke, 2002). Our hypothesis was that survival of EHEC will be reduced after SCFA stress. To test this hypothesis, we employed a plate count method to assess whether E. coli O157:H7 86-24 was capable of survival after SCFA exposure. As predicted, in the presence of either individual treatments or a mixture of all SCFAs, E. coli O157:H7 86-24 resulted in significantly lower viabilities relative to that of the control by a factor of $1.3-1.5$. 
Butyrate and propionate treatments showed even significantly lower survival levels over the other two treatments; acetate and mixture. These observations may suggest a higher impact of butyrate and propionate treatments on the survival of the bacteria. The results of SCFA stress on the viability of E. coli O157:H7 86-24 are shown in Figure 3.6-1.

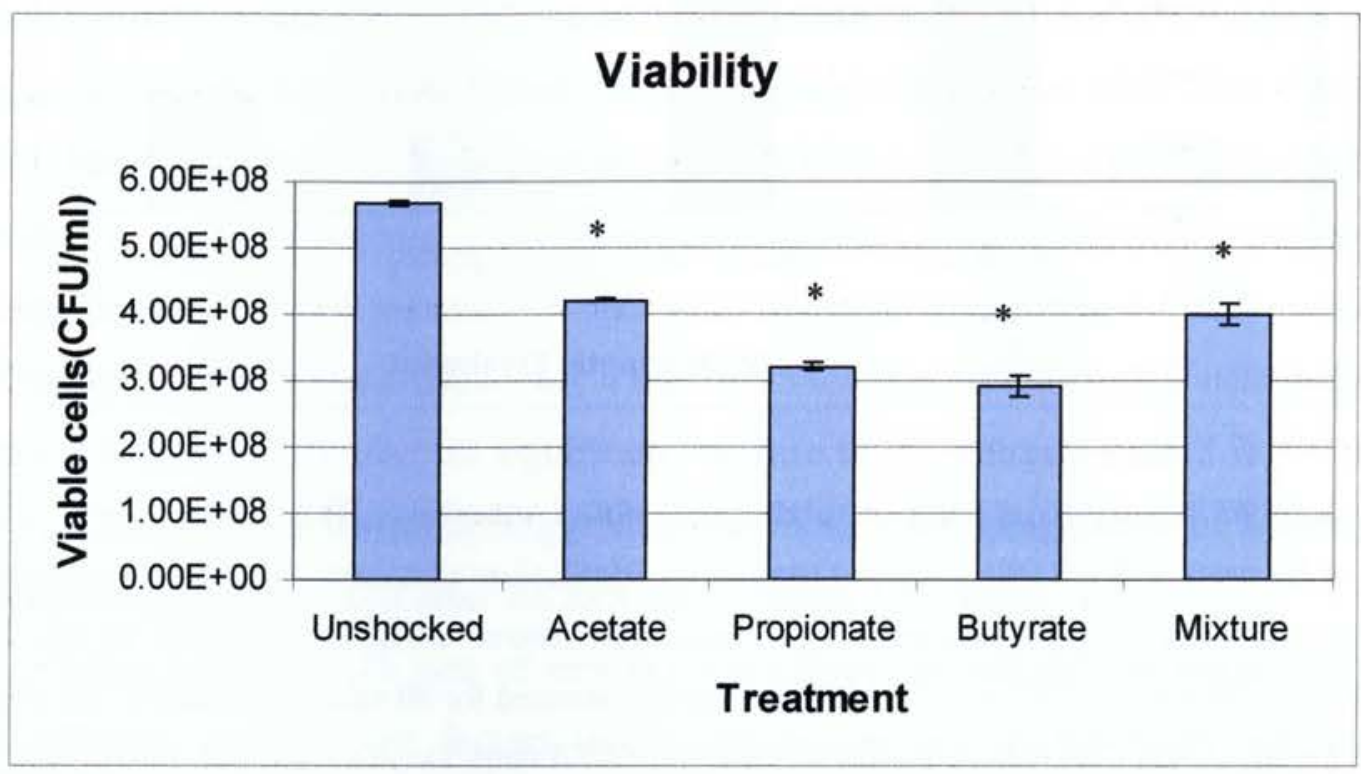

\section{Figure 3.6-1 SCFATreatment Decreases EHEC 0157:H7 86-24 Survival}

Effect of SCFA stress on E. coli O157:H7 86-24 viability was assessed by a plate count assay (n=3). This is the result of 2 independent experiments. Values are means \pm standard deviations. Short Chain Fatty Acid Treatment: $16-18 \mathrm{hr}, 37^{\circ} \mathrm{C}, 5 \% \mathrm{CO}_{2}$, Sodium Acetate $(110 \mathrm{mM})$, Sodium Propionate $(70 \mathrm{mM})$, Sodium Butyrate $(20 \mathrm{mM})$ and a Mixture: combined acetate-propionate-butyrate. [Unshocked: Control (Unshocked for 16-18 hr); Acetate: Sodium Acetate shocked for 16-18 hr; Propionate: Sodium Propionate shocked for 16-18 hr; Butyrate: Sodium Butyrate shocked for 16-18 hr); Mixture: Mixture of the above shocked for 16$18 \mathrm{hr}]$.

*Significantly different from corresponding control $(\mathrm{P}<0.05)$

\subsection{Short Chain Fatty Acids Stress Enhances E. coli 0157:H7 Adhesion to Human Epithelial Cells}

In order to establish infection, E. coli $\mathrm{O} 157: \mathrm{H} 7$ must overcome the short chain fatty acid stress environment of the large intestine. Since there are no previous studies that have addressed the role of SCFA on EHEC adherence capability, the present study tried to clarify whether SCFA stress has any impact on EHEC adhesion to epithelial cells. 
To judge the level of adherence of E. coli $\mathrm{O} 157: \mathrm{H} 7$ strains to human epithelial cells, adhesion assays before and after SCFA stress were conducted. Quantification of bacterial adherence to human intestinal cells was determined by plate count assay as previously described by de Jesus et al. (2005). The effect of different SCFAs was examined, individually and in combination (acetate, propionate, butyrate and a mixture of above). EHEC 86-24 (O157:H7) strains were assesed with either human colonic Caco-2 or nonintestinal HEp-2 epithelial cells for 4 hours. No significantly different bacterial adherence on epithelial cells was produced with either acetate or propionate treatments relative to unshocked control.

However, adhesion of butyrate and SCFA mixture treated EHEC 86-24 to epithelial cells (either HEp-2 or Caco-2 cells) was significantly higher than that of untreated control EHEC 86-24. Adhesion enhancement factors ranged from 1.5 - 1.8 for butyrate and 1.6 3 for SCFA mixture (Fig 3.7-1; Figure 3.8-1 and Table 2 Supplementary Data). It is possible that changes in bacterial adherence to eukaryotic cells after treatment could depend on the concentration of SCFA used. Future research should examine the role of concentration of SCFA in the adhesion of treated bacteria. 


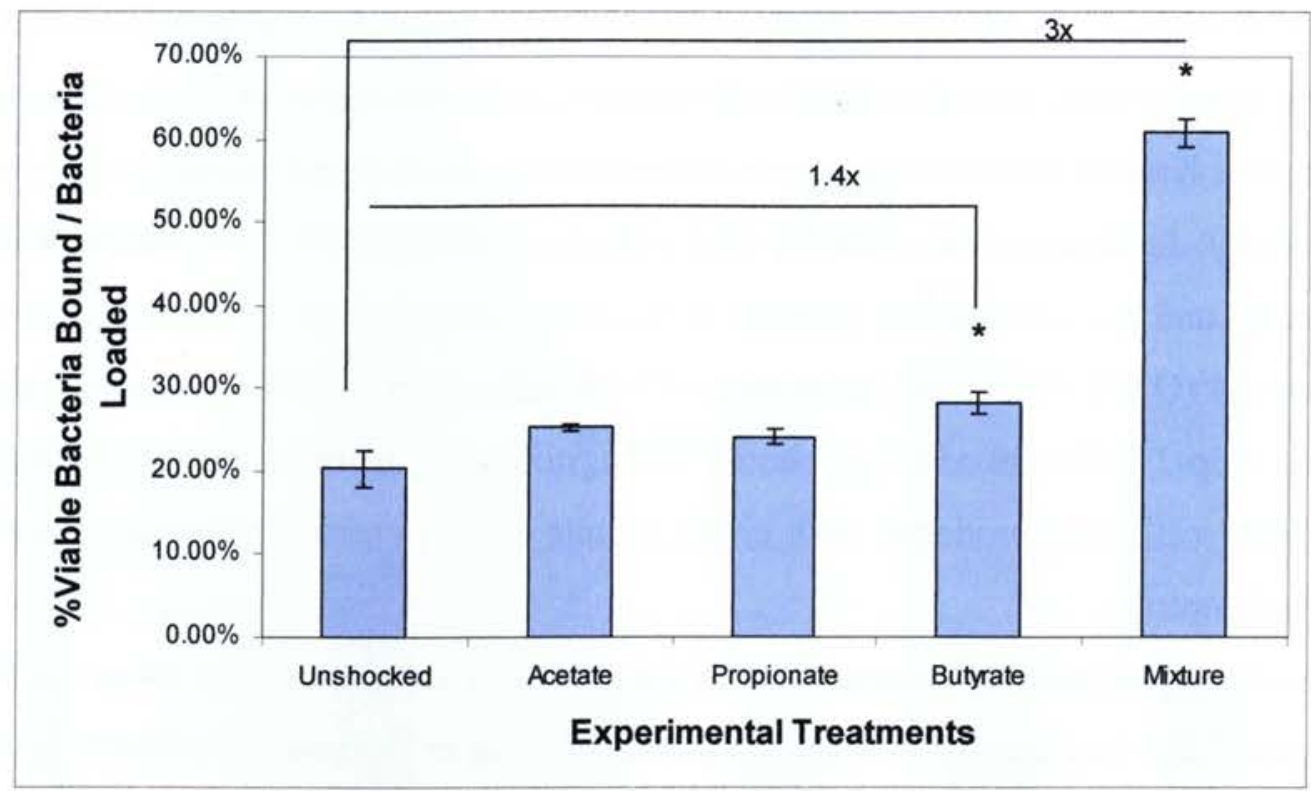

Figure 3.7-1 SCFA Shocked E. coli 0157:H7 86-24 Show Enhanced Adhesion to HEp-2 Cells Effect of SCFA stress onE. coli O157:H7 8624 in adhesion to HEp-2 cells (MOI range 34-60) was assessed by plate count assay $(n=3)$. Results representative of 2 independent experiments are shown and the values are means \pm standard deviations. Short Chain Fatty Acid Stress Treatment: $16 \mathrm{hr}, 37^{\circ} \mathrm{C}$, Acetate: $110 \mathrm{mM}$; Propionate: $70 \mathrm{mM}$; Butyrate: $20 \mathrm{mM}$; Mixture of combined acetate-propionate-butyrate: $\sim 90$ $\mathrm{mM}$

*Significantly different from corresponding control $(\mathrm{P}<0.05)$;

\subsection{Erythromycin Treatment Abrogates Short Chain Fatty Acids Host Adhesion Enhancement}

To determine whether the adhesion enhancement observed after SCFA stress was a result of increased expression of adhesins, erythromycin, below the MIC, was used to inhibit protein synthesis (de Jesus et al., 2005). Following stress, all treatments were incubated at room temperature for 30 minutes with or without $0.06 \mathrm{mg} / \mathrm{mL}$ erythromycin. The adhesion capability of E. coli O157:H7 86-24 after SCFA stress exposure to HEp2 cells was examined in the presence of erythromycin. Pretreatment of bacteria with erythromycin significantly decreased the adhesive capability of E. coli $\mathrm{O} 157: \mathrm{H} 7$ to Caco2 cells (Figure 3.8-1). 


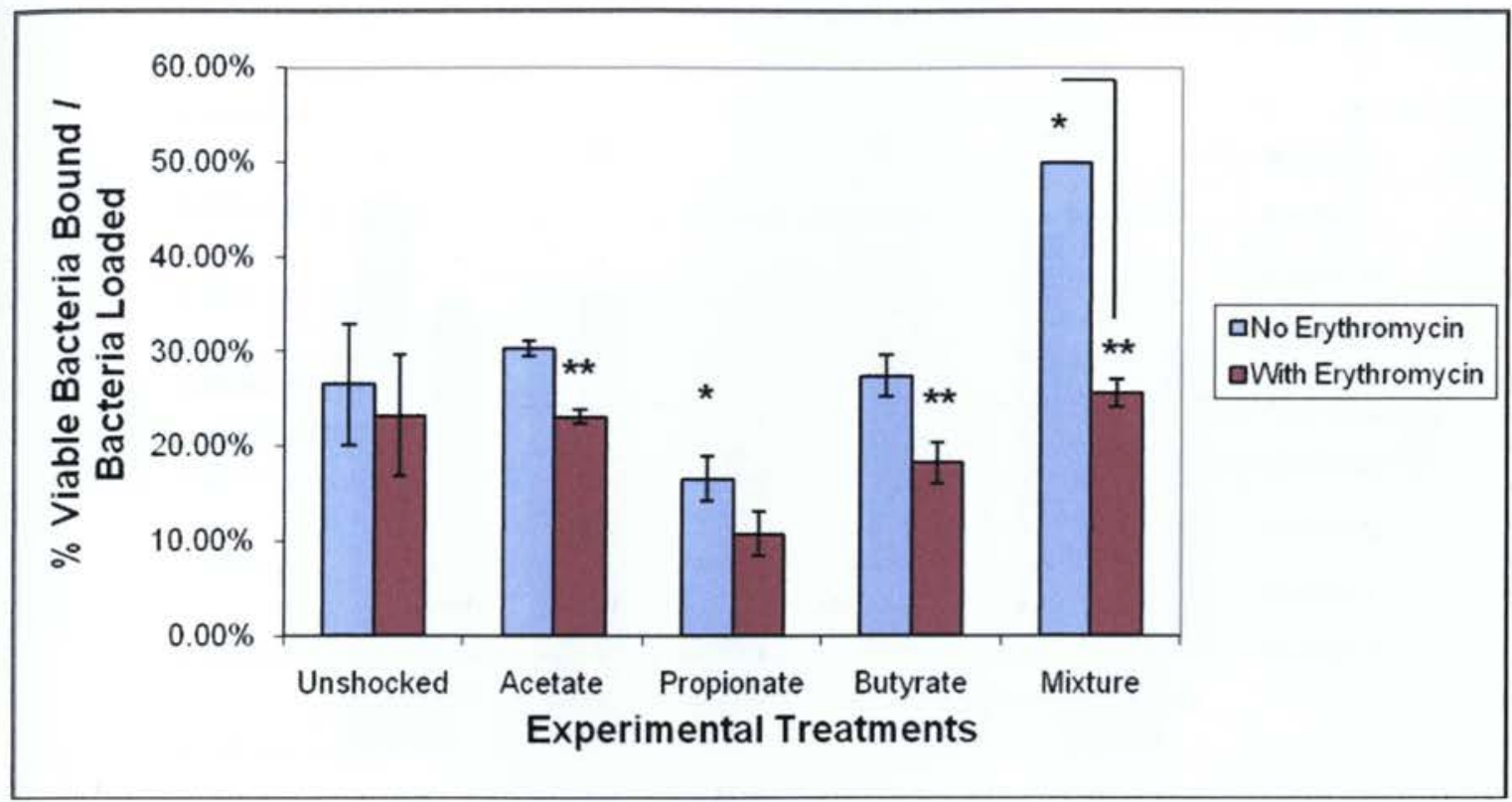

Figure 3.8-1 Erythromycin Treatment Reduces SCFA Host Adhesion Enhancement to Caco-2 Cells The effect of erythromycin pretreatment on SCFA stressed E. coli O157:H7 in adhesion to HEp-2 cells (MOI range 22-60) was assessed by plate count assay $(n=6)$. Values are means \pm standard deviations.

* Significantly different from unshocked control $(\mathrm{p} \leq 0.05)$.

** Significantly different from corresponding SCFA control without erythromycin $(\mathrm{p} \leq 0.05)$;

\subsection{The Effect of Bile Salts and Short Chain Fatty Acids Stress on $E$. coli 0157:H7 86-24 Growth during Infection Time}

The enhanced adhesion of BS and SCFA stressed E. coli $\mathrm{O} 157: \mathrm{H} 7$ to tissue culture cells may be due to increased growth that may arise after BS or SCFA stress. To test this possibility, control adhesion assays were employed; E. coli O157:H7 86-24 was exposed to either BS or SCFA and then incubated, but without epithelial cells, simulating the conditions of the adhesion assays previously described. After $4 \mathrm{~h}$ incubation at $37^{\circ} \mathrm{C}$ with $5 \% \mathrm{CO}_{2}$ bacteria were plated out and the next day counted (Figure 3.9-1 and Figure 3.92). Three of the treatments (glycholate, deoxycholate and ursocholate) showed significantly higher final viability compared to the control. These results may point out the possibility that the enhanced adhesion of BS stressed E. coli O157:H7 86-24 might be due to increased growth after stress. 


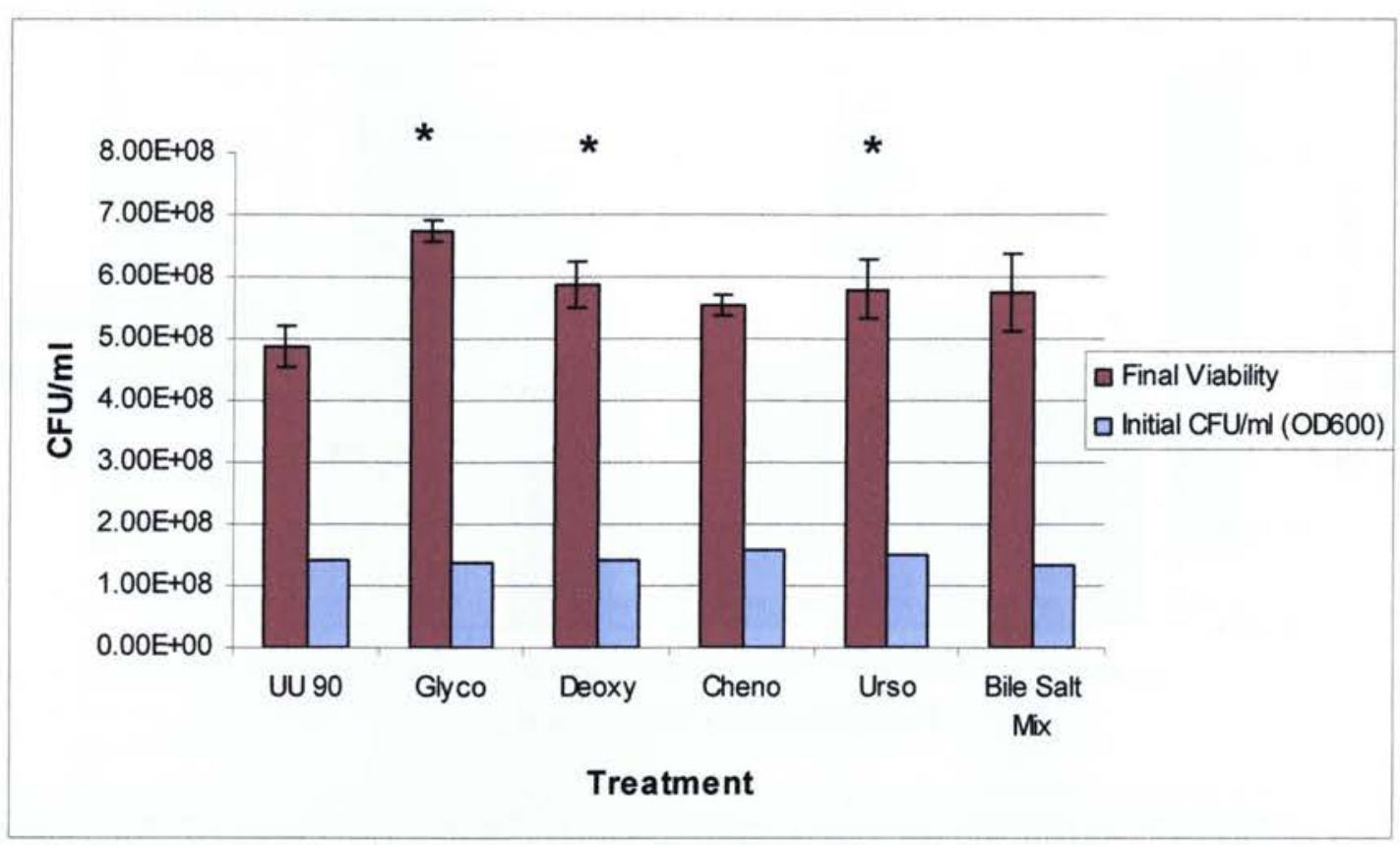

Figure 3.9-1 The Effect of BS Treatment on E. coli O157:H7 86-24 Viability during 4 h Incubation The effect of BS Treatment on E. coli $\mathrm{O} 157: \mathrm{H} 786-24$ viability was assessed by plate count assay $(\mathrm{n}=3$ for each experiment) Initial viability: initial amount of bacteria loaded. Final viability: Culturability after $4 \mathrm{~h}$ incubation at $37^{\circ} \mathrm{C}$ and $5 \% \mathrm{CO} 2$. The results are representative of 2 independent experiments and the values are means \pm standard deviations. BS Stress Treatment: $90 \mathrm{~min}, 37^{\circ} \mathrm{C}, 5 \% \mathrm{CO} 2$ Cheno/Deoxy/Urso/Glyco: 2.5 mM; BS Mix: 0.15\% w/v. [Unshocked: Control (Unshocked for 90 minutes); Cheno: Chenodeoxycholate shocked for 90 minutes; Deoxy: Deoxycholate shocked for 90 minutes; Glyco: Glycocholate shocked for 90 minutes); Urso: Ursodeoxycholate shocked for 90 minutes; Mixture: BS Mixture shocked for 90 minutes].

*Significantly different from corresponding control $(\mathrm{P}<0.05)$

The growth of SCFA stressed E. coli $\mathrm{O} 157: \mathrm{H} 7$ 86-24 during $4 \mathrm{hr}$ incubation time showed a significant decrease in viability for all treatments in comparison to the control. These results are consistent with previous viability results of EHEC 86-24 after exposure to SCFAs and indicate that increased adhesion of SCFA stressed E. coli O157:H7 86-24 is not mediated through increased growth during infection time. 


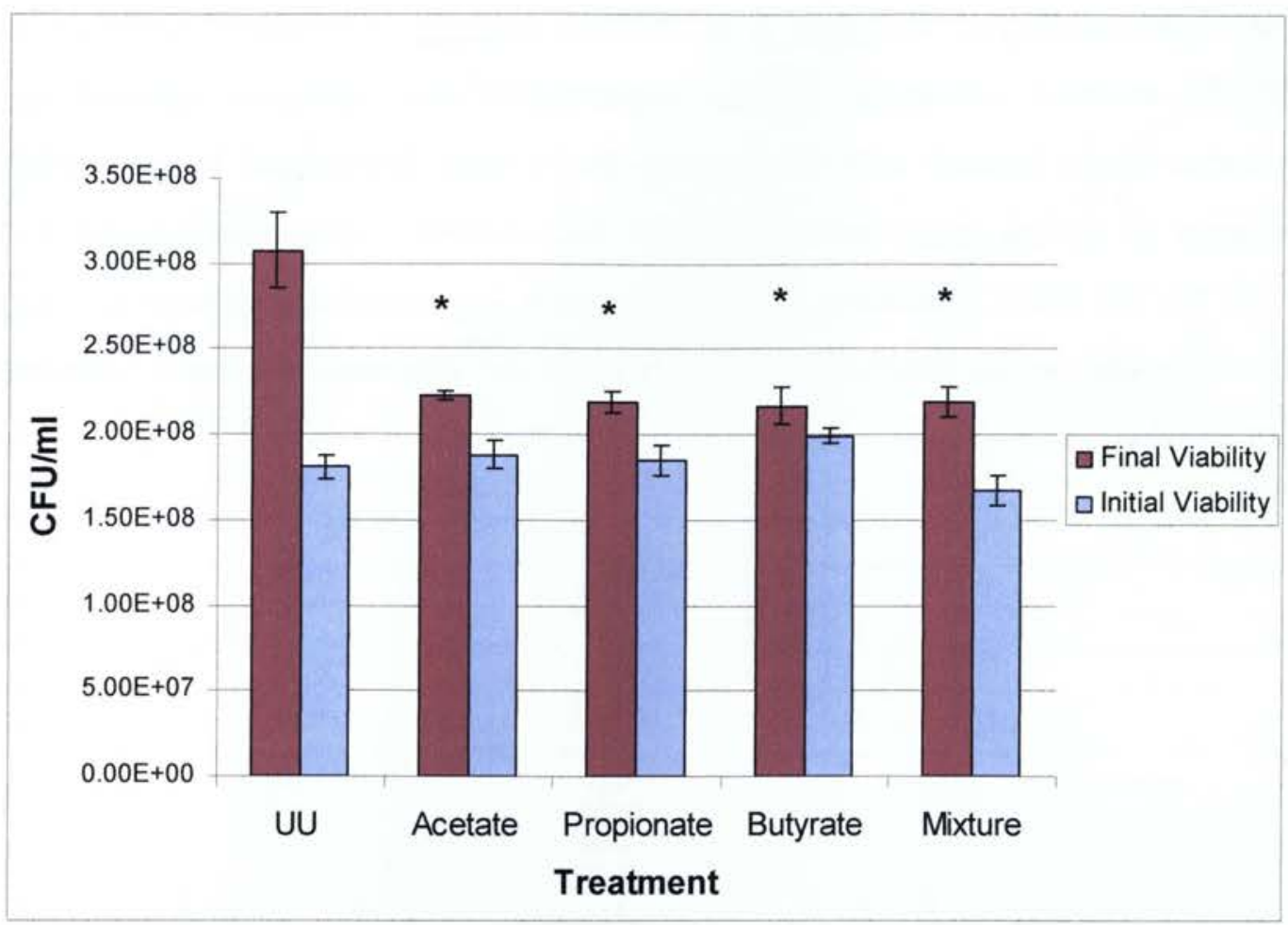

Figure 3.9-2 The Effect of SCFA Stress on E. coli O157:H7 86-24 Viability during $4 \mathrm{~h}$ Incubation The effect of BS Treatment on E. coli O157:H7 86-24 viability assessed by plate count assay ( $\mathrm{n}=3$ for each experiment). Initial viability: initial amount of bacteria loaded. Final viability: Culturability after $4 \mathrm{~h}$ incubation at $37{ }^{\circ} \mathrm{C}$ and $5 \% \mathrm{CO} 2$. The results are representative of 2 independent experiments and the values are means \pm standard deviations. Short Chain Fatty Acid Stress Treatment: $16 \mathrm{hr}, 37^{\circ} \mathrm{C}$, Acetate: 110 $\mathrm{mM}$; Propionate: 70mM; Butyrate: $20 \mathrm{mM}$; Mixture of combined acetate-propionate-butyrate: $\sim 90 \mathrm{mM}$ * Significantly different from corresponding untreated $(\mathrm{P}<0.05)$

\subsection{No Change in Secreted and Periplasmic VT Production after BS Treatment}

The effect of BS stress on both the verotoxin secreted, as well as that retained in the periplasmic space of 86-24 was examined using the vero cell cytotoxicity assay. The bacteria were subjected to either BS or SCFA stress, then normalized based on prior viability experiments and grown overnight in rich media to induce verotoxin production. Various dilutions of the supernatant filtrates and periplasmic extracts were incubated with semi-confluent monolayers of vero cells for $2-3$ days at $37^{\circ} \mathrm{C}$ with $5 \% \mathrm{CO}_{2}$. The remaining live cells were fixed with $2 \%$ formalin and stained with crystal violet. The 
crystal violet staining of verocells is an indirect measure of levels of toxin produced. Levels of secreted verotoxin (in the supernatant) and verotoxin retained in the periplasmic space formed with BS-stressed 86-24 were determined by measuring the absorbance of crystal violet with an ELISA plate reader (Figures 3.10-1 and 3.10-2). None of the BS stress treatments appeared to show any significant change in verotoxin production either in the periplasmic extracts or in the supernatant filtrates compared to the untreated controls. 

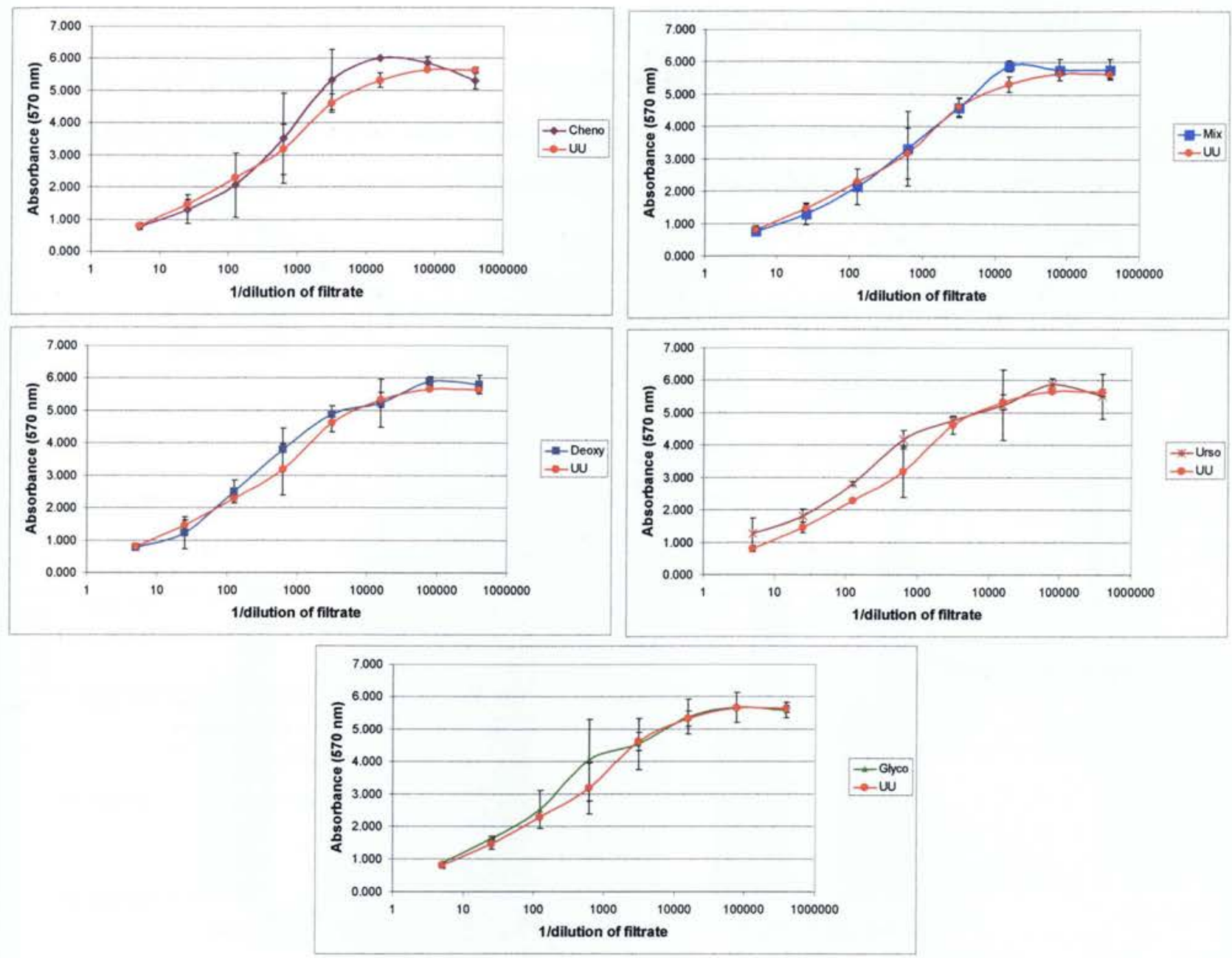

Figure 3.10-1 Secreted Verotoxin Production of E. coli O157:H7 86-24 after Exposure to BS

The effect of BS treatment on the verotoxin secreted in the supernatant by E. coli O157:H7 86-24 was measured by the vero cell cytotoxicity assay. Results are expressed as the mean absorbance \pm standard deviations (corresponding to the remaining live vero cells) versus the 1 /dilution of verotoxin in the supernatant and are representative of two independent experiments. Experimental treatments: Unshocked: Control (Unshocked for 90 minutes); Cheno: Chenodeoxycholate shocked for 90 minutes; Deoxy: Deoxycholate shocked for 90 minutes; Glyco: Glycocholate shocked for 90 minutes); Urso: Ursodeoxycholate shocked for 90 minutes; Mixture: BS Mixture shocked for 90 minutes]. 

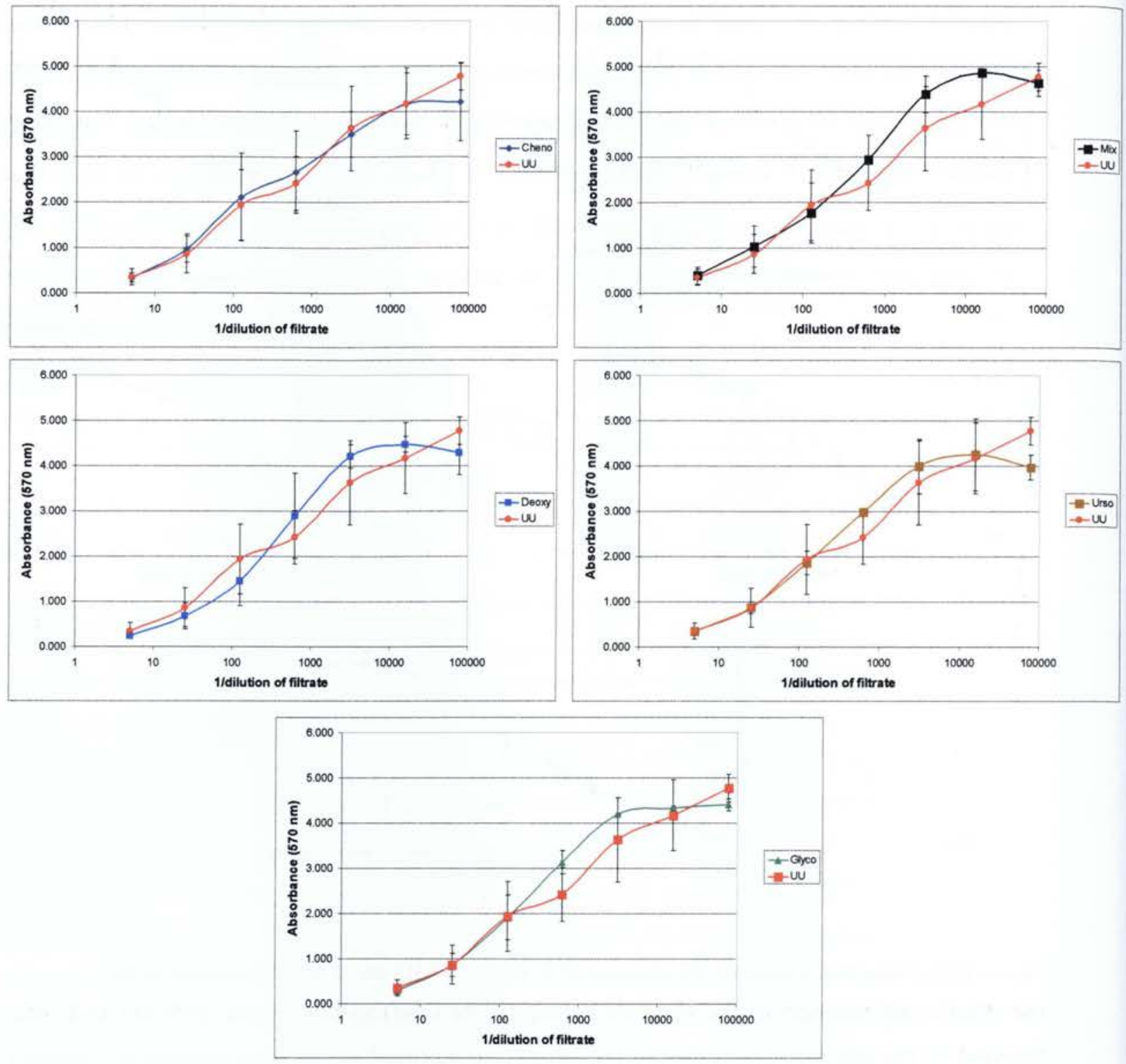

Figure 3.10-2 Periplasmic Verotoxin Production of $E$. coli O157:H7 86-24 after Exposure to BS

The effect of BS treatment on the verotoxin produced in the periplsmic space by $E$. coli $\mathrm{O} 157: \mathrm{H} 786-24$ was measured by the vero cell cytotoxicity assay. Results are expressed as the mean absorbance \pm standard deviations (corresponding to the remaining live vero cells) versus the 1/dilution of verotoxin in the supernatant and are representative of two independent experiments. Experimental treatments: Unshocked: Control (Unshocked for 90 minutes); Cheno: Chenodeoxycholate shocked for 90 minutes; Deoxy: Deoxycholate shocked for 90 minutes; Glyco: Glycocholate shocked for 90 minutes); Urso: Ursodeoxycholate shocked for 90 minutes; Mixture: BS Mixture shocked for 90 minutes]. 
To confirm that these experimental results were not the product of significantly different initial bacteria inoculums, the initial and final $\mathrm{CFU} / \mathrm{mL}$ for each treatment prior to and after overnight growth, were measured (Figure 3.10-3). Results demonstrate that initial and final bacteria concentrations were the same across all treatments.

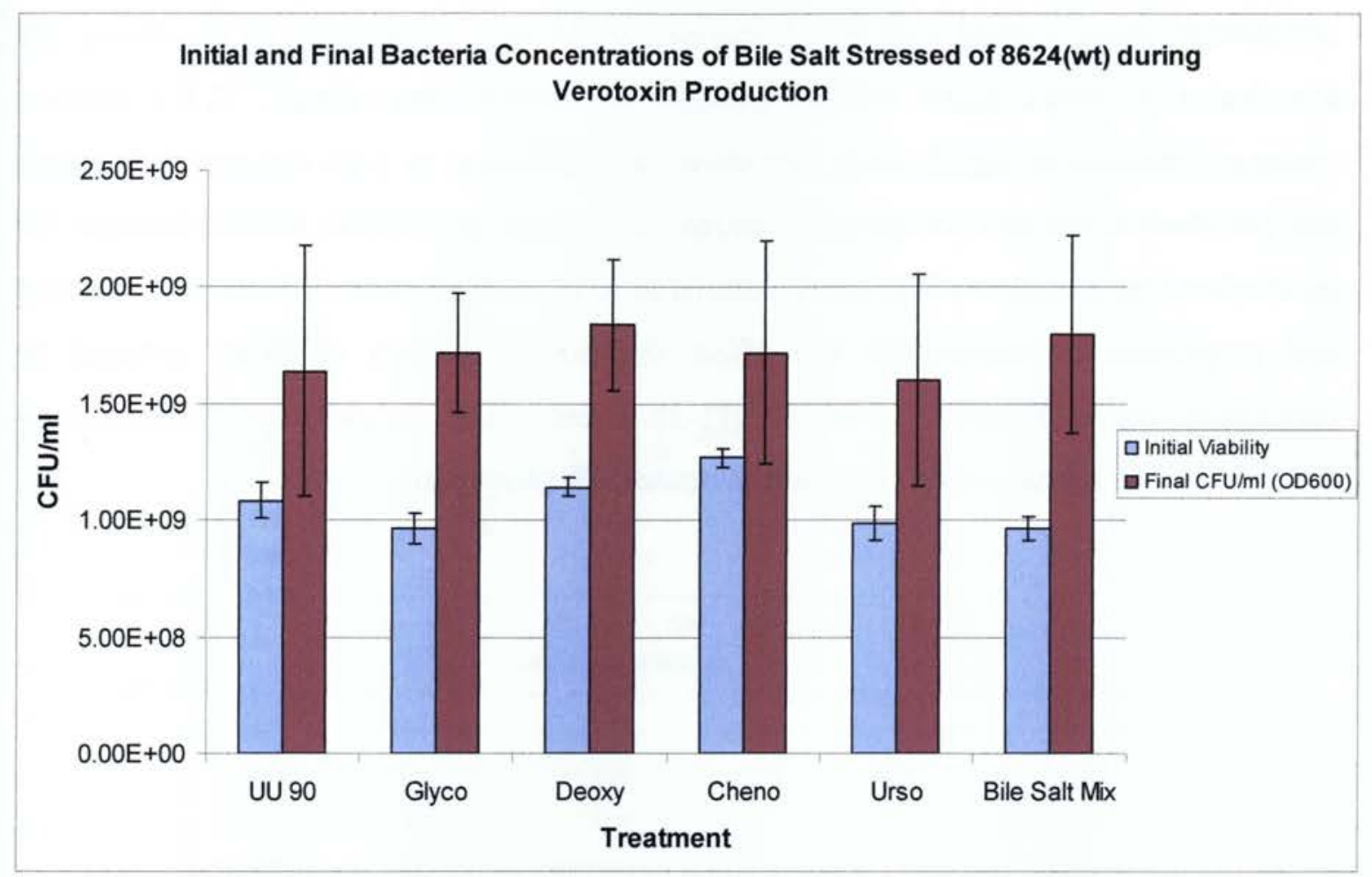

Figure 3.10-3 Initial and Final Bacteria Concentrations of BS Treated $E$. coli O157:H7 86-24 during

\section{Verotoxin Production}

The initial and final bacteria concentrations of BS treated E. coli O157:H7 86-24 were measured either by plate count or $\mathrm{OD}_{600}$ to confirm that the initial inoculums and growth during verotoxin production were the same across all treatments. After BS exposure, a plate count assay $(n=3)$ was completed to determine the initial $\mathrm{CFU} / \mathrm{ml}$ for each treatment. Following overnight growth, the $\mathrm{OD}_{600}$ of bacterial cells after each treatment was taken and converted to a final $\mathrm{CFU} / \mathrm{mL}$ using a standard curve previously completed. $\mathrm{OD}_{600}$ results are from two independent experiments and the results are the means \pm standard deviations. For both final and initial bacteria concentrations, $\mathrm{P}<0.05$. 


\subsection{VT Levels Increased after SCFA Mixture}

A similar experiment to investigate the verotoxin secreted into the supernatant and that retained in the periplasmic space were conducted for bacteria exposed to SCFA. Levels of secreted verotoxin (in the supernatant) and verotoxin retained in the periplasmic space formed with SCFA-stressed 86-24 were determined by measuring the absorbance of crystal violet with an ELISA plate reader. Interestingly, SCFA mixture treatment resulted in significantly increased Stx production in both supernatant filtrates and periplasmic extracts compared to untreated controls. In contrast, acetate induced Stx production was significantly dropped compared to unshocked control. However, butyrate and propionate treatments did not affect the Stx production of either secreted or periplasmic levels (Figures 3.11-1 and 3.11-2). These results suggest that SCFAs mixture that mimics the human colon condition increases VT production. 

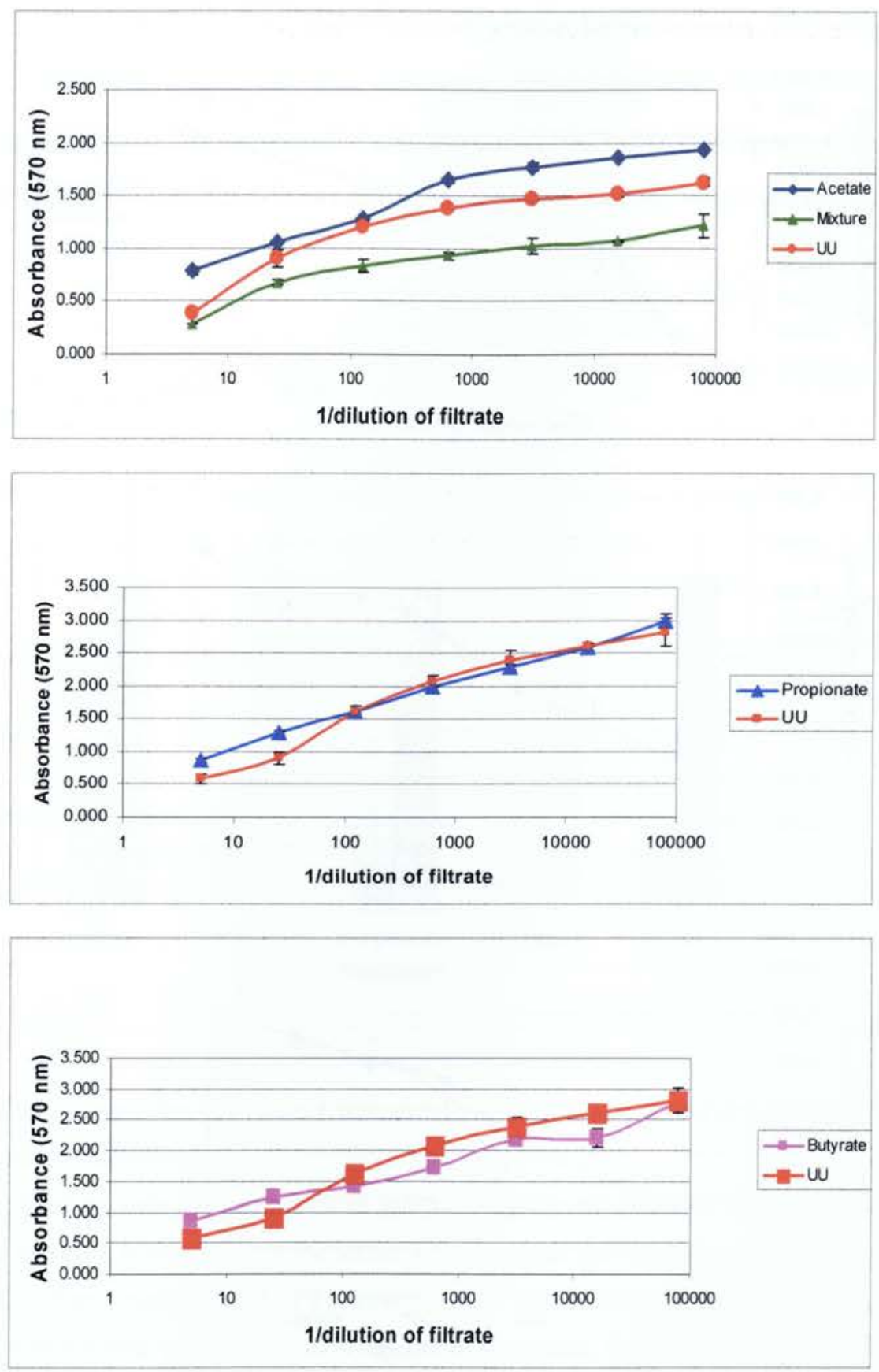

Figure 3.11-1 Secreted Verotoxin Production of SCFA Stressed E. coli O157:H7 86-24

The effect of SCFA stress on the verotoxin secreted in the supernatant by E. coli O157:H7 86-24 was measured by the vero cell cytotoxicity assay. Results are expressed as the mean absorbance \pm standard deviations (corresponding to the remaining live vero cells) vs. the 1 /dilution of verotoxin in the supernatant and are representative of two independent experiments. Experimental treatments: Short Chain Fatty Acid Stress Treatment: $16 \mathrm{hr}, 37^{\circ} \mathrm{C}$, Acetate: $110 \mathrm{mM}$; Propionate: $70 \mathrm{mM}$; Butyrate: $20 \mathrm{mM}$; Mixture of combined acetate-propionate-butyrate: $\sim 90 \mathrm{mM}$ 

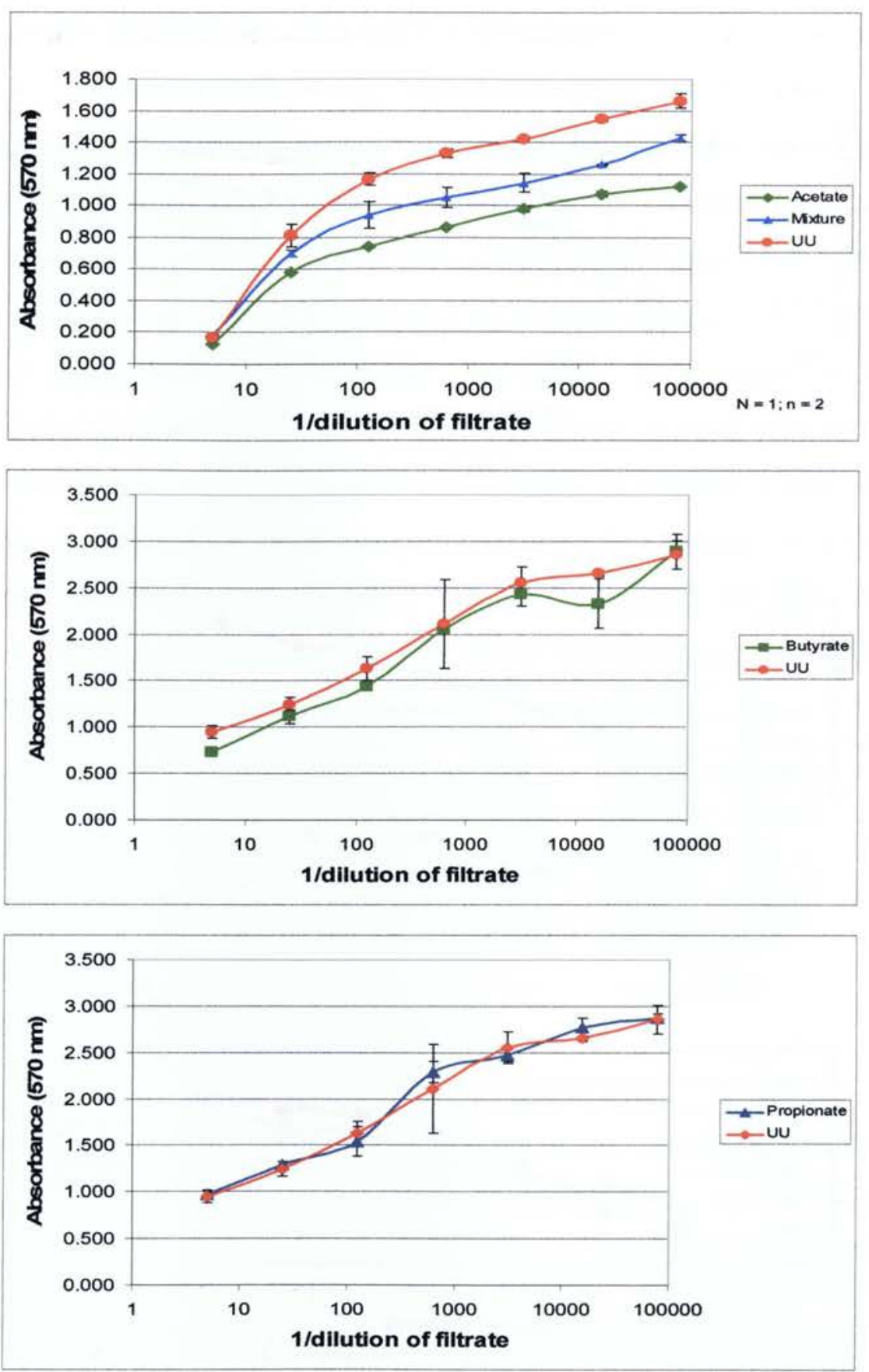

Figure 3.11-2 Secreted Verotoxin Production of SCFA Stressed E. coli O157:H7 86-24

The effect of SCFA stress on the verotoxin produced in the periplsmic space by E. coli O157:H7 86-24 was measured by the vero cell cytotoxicity assay. Results are expressed as the mean absorbance \pm standard deviations (corresponding to the remaining live vero cells) versus. the $1 /$ dilution of verotoxin in the supernatant and are representative of two independent experiments. Experimental treatments: Short Chain Fatty Acid Stress Treatment: $16 \mathrm{hr}, 37^{\circ} \mathrm{C}$, Acetate: $110 \mathrm{mM}$; Propionate: 70mM; Butyrate: $20 \mathrm{mM}$; Mixture of combined acetate-propionate-butyrate: $\sim 90 \mathrm{mM}$ 
Again, to confirm that these experimental results were not the product of significantly different initial bacteria innocula, the initial and final CFU/mL for each treatment prior and after overnight growth were measured (Figure 3.11-3). Results demonstrate that initial and final bacteria concentrations were the same across all treatments.

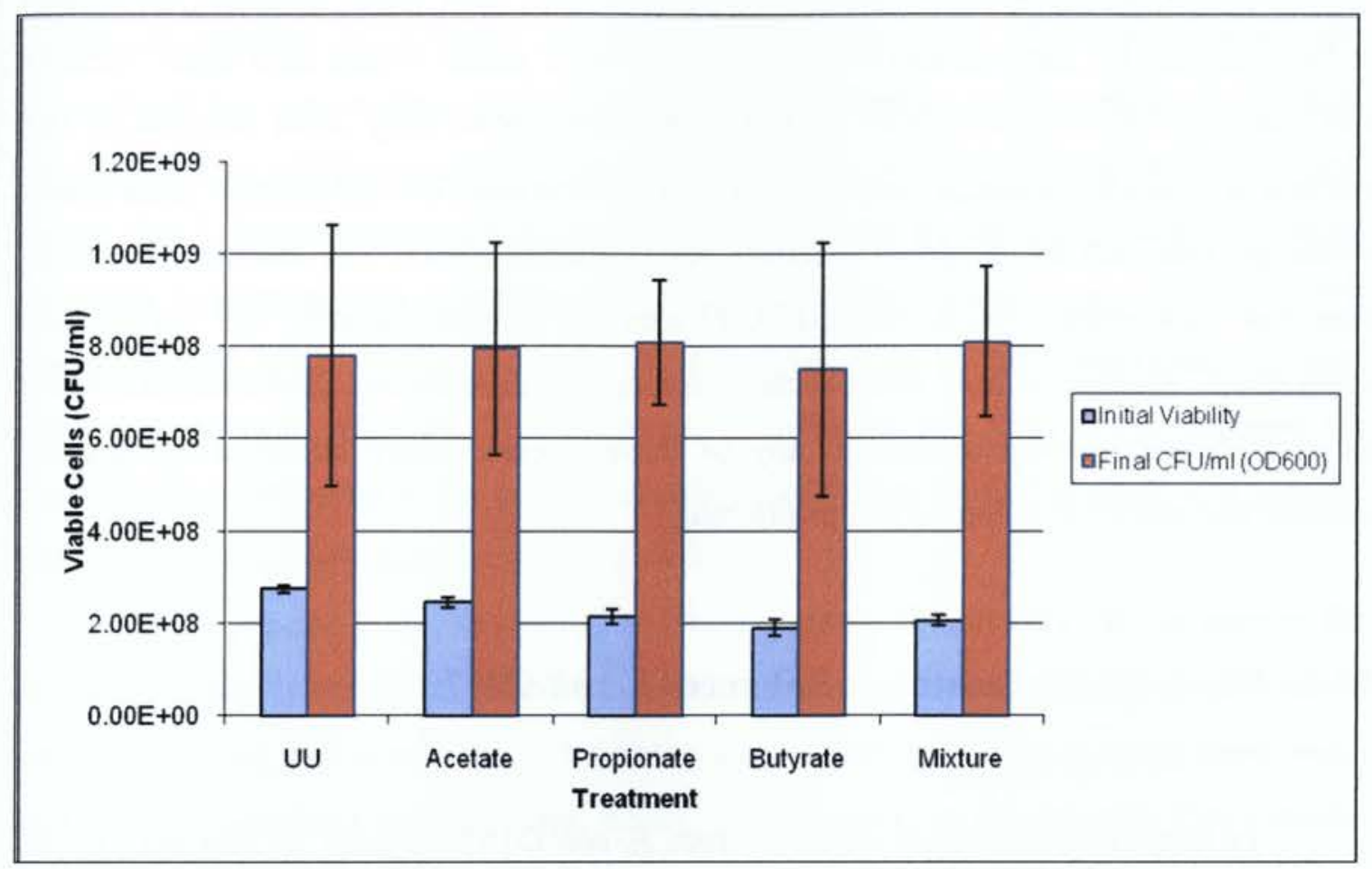

Figure 3.11-3 Initial and Final Bacteria Concentrations of SCFA Stressed E. coli O157:H7 86-24

\section{during Verotoxin Production}

The initial and final bacteria concentrations of SCFA treated E. coli O157:H7 86-24 were measured either by plate count or $\mathrm{OD}_{600}$ to confirm that the initial inoculums and growth during verotoxin production were the same across all treatments. After SCFA stress, a plate count assay $(n=3)$ was completed to determine the initial $\mathrm{CFU} / \mathrm{ml}$ for each treatment. Following overnight growth, the $\mathrm{OD}_{600}$ of each treatment was taken and converted to a final $\mathrm{CFU} / \mathrm{mL}$ using a standard curve previously completed. $\mathrm{OD}_{600}$ results are from two independent experiments and the results are the means \pm standard deviations. For both final and initial bacteria concentrations, $\mathrm{P}<0.05$. 


\section{Discussion}

\subsection{Effect of Intestinal Stress on E. coli O157:H7 Survival: Summary}

The ability to adapt to environmental stresses, such those imposed by human hosts, is critical for survival and proliferation of EHEC strains within their hosts (Merrell and Camilli, 2002). When these bacteria enter their hosts they must respond to the extreme and rapidly changing conditions such as gastric acid, BS and organic acids which challenge their ability to grow (Merrell and Camilli, 2002). The effects of human intestinal tract stresses on E. coli $\mathrm{O} 157: \mathrm{H} 7$ survival and pathogenicy are not entirely understood. Studies on how these bacteria respond to these stress conditions will allow us to understand how exposure to any of these stressors may influence subsequent survival and ability to colonize and proliferate.

\subsubsection{Bile Salt Mix Treatment Enhances $E$. coli O157:H7 Survival}

To survive in the human intestinal tract, E. coli $\mathrm{O} 157: \mathrm{H} 7$ must tolerate high levels of BSs in the upper small intestine at physiological concentrations of between $0.1-1.3 \%$ (Pumbwe et al., 2007). BSs have detergent like properties which can permeabilize bacterial membranes and can lead to membrane collapse (Begley et al., 2005). Several pathogens including, E. coli $\mathrm{O} 157: \mathrm{H} 7$, have adapted mechanisms to evade the antimicrobial activity of BSs. Various effects of BSs on bacteria have been observed after exposure which depend on factors including the type, length and severity of the stress, the growth phase of the organism, and the ability of different strains to survive the encountered stress. For example de Jesus and colleagues (2005) reported significant reductions in viability of BS stressed EPEC relative to that of untreated controls. Another study by Pumbwe et al. (2007) demonstrated that B. fragilis exposed to $0.15 \%$ BS mixture extracted from porcine bile had a longer lag phase, and slower growth rate compared with bacteria grown with media alone. 
In contrast Pace et al. (1997) showed that pathogenic $V$. parahaemolyticus strains have the ability to survive to levels $0.5-1 \log$ higher on the agar containing $0.15 \%$ oxgall bile compared with strains grown in the presence of no bile. Little difference was observed in survival of non-virulent strains of the bacterium with $0.15 \%$ bile. However, when pathogenic strains were grown on plates containing higher concentrations of bile $(1.5 \%)$, it resulted in reduced survival. Interestingly, this effect was particularly pronounced for non-virulent strains suggesting that virulent strains appear to be more resistant to bile inhibitory activity (Pace et al., 1997). Additionally, other studies have shown that L. monocytogenes is capable of colonizing the human gall bladder indicating an ability to tolerate high concentrations of bile (Allerberger and Guggenbichler, 1989; Briones et al., 1992; Begley et al., 2002). The role of BS in this context has not been fully explored in E. coli $\mathrm{O} 157: \mathrm{H} 7$ strains, and therefore in this study we investigated the contribution of BS stress to the survival capability of the bacteria. Therefore, it is possible that BS exposure might increase EHEC survival.

In the present study, the ability of mid-log phase E. coli O157:H7 to survive BS exposure at physiological concentrations was examined. BS treated cells were found to survive better on agar compared with untreated cells, after exposure to either three major individual components of BS (cheno, deoxy, glycocholate) or BS mixture. The viability of BS treated EHEC increased 1.2 - 1.4 fold relative to that of untreated EHEC ( $p \leq$ 0.05). These findings are not surprising as the most commonly-used selective media for detection of E. coli O157:H7 is sorbitol- MacConkey agar (SMAC medium) which contains BS (March and Ratnam, 1986).

Interestingly, studies of the impact of acid stress on E. coli $\mathrm{O} 157: \mathrm{H} 7$ revealed significantly lower survival of the cells compared to the untreated controls (House et al., 2006). It is possible that BS present in the small intestine may favour growth of EHEC to facilitate recovery from the acid stress of gastric passage and proliferation prior to entry and colonization in the large intestine. 


\subsubsection{Short Chain Fatty Acids Treatment Reduces $E$. coli O157:H7 Survival}

E. coli $\mathrm{O} 157: \mathrm{H} 7$ that survive and transit the small intestine enter the large intestine where they encounter stresses associated with SCFA. The SCFA concentrations in the human colon, the major site of EHEC colonization, are $110 \mathrm{mM}, 70 \mathrm{mM}$ and 20 $\mathrm{mM}$ for acetate, propionate and butyrate, respectively (Cummings et al., 1987; Macfarlane et al., 1992; Lawhon et al., 2002). It is well documented that SCFA have antimicrobial activity, and they are widely used as preservatives in food products (Maurer et al., 2005). Organic acids are able to exibit antimicrobial effect by penetrating the bacterial membrane and dissociation into anions in the cytoplasm. Consequently this results in an increase of the internal osmotic pressure and a depletion of cellular energy to maintain a near neutral pH (Ricke, 2003) Various SCFAs including acetate, propionate, and butyrate are produced by the microbial flora of the large intestine such as Lactobacillus sp., Bifidobacterium sp. and Bacteroides sp. These resident flora can enhance host defence by producing SCFA that in turn create an unfavorable environment for enteric pathogens ( $\mathrm{Lu}$ and Walker, 2001). On the other hand, enteric pathogens including E. coli, Shigella and Salmonella appear to only generate acetate. Acetate can pass through the bacterial membrane into the cytoplasm where it dissociates, generates a large accumulation of anions and lower internal pH (Maurer et al., 2005). Growth inhibition occurs as a result of lower $\mathrm{pH}$ and the ability of anions to inhibit metabolism (Salmond et al., 1984). This evidence was supported by recent studies which revealed that acetate concentrations increase as bacterial density increases (Arnold et al., 2001; Kirkpatrick et al., 2001). Other studies have also shown that SCFAs can have inhibitory effects on Salmonella growth and colonization of gastrointestinal tract. However, there are no previous studies that have addressed the role of SCFA on EHEC pathogenesis. We reasoned that exposure to SCFA may decrease EHEC survival. Because the gastrointestinal tract contains a mixture of acetate, propionate and butyrate, individual SFCAs as well as a mixture of SCFAs were investigated for their affects on EHEC. The survival of the cells with exposure to acetate, propionate, butyrate and the mixture was significantly lower than untreated cells by a factor of $1.3-1.5$. Here we show for the first 
time that a mixture of SCFAs predicted to be similar to that present in the human large intestine significantly decreased viability relative to unstressed cells by a factor of 1.3 .

\subsection{Bile Salt Mix Treatment Enhances E. coli 0157:H7 Adhesion to Epithelial Cells}

The ability of many gastrointestinal bacteria including E. coli $\mathrm{O} 157: \mathrm{H} 7$ to adhere to host is essential for colonization and requires survival through a wide range of changing environments in the human digestive tract, including BS in the small intestine followed by SCFA in the large intestine. Only those pathogens that evade all these host defenses are able to cause infection. Many enteric pathogens have evolved several complex defense mechanisms, which are induced under different environmental conditions dependant on many factors such as growth phase, the type and the severity of the stress encountered (Lu \& Walker, 2001). These important stress responses of $E$. coli O157:H7 enhance virulence and correlate to the low infectious dose of approximately 100 organisms (de Jesus et al., 2005). BS have been shown to play a role in host cell adhesion for several gastrointestinal pathogens including Shigella flexneri, Vibrio parahaemolyticus and Listeria monocytogenes (Pope et al., 1995; Pace et al., 1997; Begley et al., 2002). Enteropathogenic E. coli which is a related pathogen has also been reported to show enhanced host-cell adhesion following exposure to BS stress (de Jesus et al., 2005). Host adhesion of typical EPEC was significantly enhanced by a factor of 1.3 to 2.6 after BS stress. The observed enhanced adhesion was dependent on the different culture conditions employed. Similarly, Torres et al. (2007) identified that BS increase expression of the afimbrial adhesin (LDA) in atypical EPEC that mediates binding to tissue-cultured cells in a diffuse adherence pattern of aEPEC. Another study by Maroncle et al. (2006) reported that Klebsiella pneumoniae host-cell attachment was significantly increased, mounting a 2 -fold higher adhesion than bacteria cultured without bile. The results reported by Pumbwe and colleagues (2007) showed that BS also contribute to enhanced colonization of $B$. fragilis through increased bacterial co-aggregation, bacterial host adhesion, biofilm formation and antimicrobial resistance. 
In addition, research by Hung et al. (2006) has shown that the gastrointestinal pathogen, $V$. cholerae had a significant increase in biofilm formation in response to bile acids.

In this study, various adhesion assays were performed with human epithelial cell lines derived from adenocarcinoma including HEp-2 (laryngeal carcinoma cells) and Caco-2 (colonic adenocarcinoma cells). However, the use of tissue cells that are cultured has the limitation of obtaining approximative results compared with in vivo models.

Regardless of the epithelial cell lines used (either HEp-2cells or Caco-2 cells) the E. coli O157:H7 86-24 strains exposed to BS for $90 \mathrm{~min}$, resulted in significantly enhanced adhesion to host cells after glycocholate and BS mixture treatments compared to the untreated controls. Even though we eliminated the role of several predominant individual components of bile salts in enhanced adhesion, however we could not rule out the role of other additional components of the bile complex such as taurocholate, cholate, cholesterol and phospholipids. Even though the viabilities for these treatments were not significantly different relative to the control cells, E. coli $\mathrm{O} 157: \mathrm{H} 7$ strains subjected to BS treatments were able to better adhere to either HEp-2 cells or Caco- 2 cells by adhesion enhancement factors that ranged from 1.6 to 3.2 depending on treatment. In agreement with these results, light microscopy photomicrographs of infected Giemsa stained cells demonstrated as well obvious increased adherence of BS mixture and glycocholate treated E. coli $\mathrm{O} 157: \mathrm{H} 7$ as compared with untreated strains.

E. coli $\mathrm{O} 157: \mathrm{H} 7$ strain 86-24 had the highest percentage of viable bacteria bound to Caco-2 cells $(>4 \%)$ and the lowest adherence levels to HEp-2 cells $(2.4-2.6 \%)$. EHEC adherence capability after exposure to BS which was also more significantly increased to colonic Caco-2 cells (4x more) than to human non-intestinal HEp-2 cells (2x). Sherman et al. (1987) have also shown differences associated with host-cell adhesion capabilities. Adherence of clinical O157:H7 isolates to both HEp-2 and Intestinal 407 cells did not follow the same morphologicy of binding (Sherman et al., 1987). These results may suggest that EHEC mediates adherence to various epithelial cell lines through recognition of different surface receptors. 
Since the Caco-2 cell line is a colonic cell line which has characteristics of mature enterocytes and is more similar to the in vivo situation, these results may suggest that the adhesion enhancement seen after BS exposure may be more dramatic in vivo as well.

The importance of adhesion in the life style of EHEC is reflected by the high number of different structures bacteria use for adhesion (Wells et al., 2008). Interestingly, Boerlin and collegues (1999) suggested a strong association for Stx2, intimin and increased adherence on HEp-2 cells. Perhaps this synergism is one factor that may contribute to the differences among strain adhesion phenotypes and ultimately severe EHEC-induced diseases as not all O157:H7 strains produce VT2. In the present study, all of the EHEC O157:H7 8624 strains used in the adhesion assays were VT2 positive strains

Since treatment with BS enhanced the adhesion of O157:H7 86-24 cells to host cells we wanted to determine if this phenotype was the result of upregulation of the expression of adhesins, and or modifications to existing adhesins. We sought to clarify the role of protein synthesis in the enhanced adhesion phenotype by pretreatment of bacterial cells with erythromycin. Huesca et al. (1996) found that erythromycin, used at concentrations below the minimal inhibitory concentration (MIC), can inhibit bacterial protein synthesis. When BS treated E. coli $0157: \mathrm{H} 7$ 86-24 were pre-treated with erythromycin, the adhesion enhancement to HEp-2 cells was abrogated. This result suggests that the enhanced adhesion of BS stressed E. coli $\mathrm{O} 157: \mathrm{H} 7$ is likely the result of enhanced expression of existing adhesins and or expression of new adhesin(s).

The ability of EHEC to adhere to host cells and subsequently colonize the large intestine is a key virulence trait and a requirement for the production of diarrhea (required for dissemination of the bacteria) (Torres et al., 2005a). To date, intimin is the only well characterized adhesin that appears to be required for intimate adherence to host cells in vivo (Donnenberg et al., 1993; McKee et al., 1995; Torres et al., 2005b). Several proteins have been implicated as novel EHEC adhesive factors, both fimbrial and nonfimbrial, including long polar fimbriae 2, outer membrane protein A (OmpA), Iha, and Efa-1 (EHEC factor for adherence) (Nicholls et al., 2000; Tarr et al., 2000; Paton et al., 2001; Stevens et al., 2002). In addition, EspA filaments also appear to be important adhesion factors (Garmendia et al., 2005). Since BS stressed E. coli O157:H7 resulted in enhanced host cell adhesion after two of treatments (glychocolate and BS mixture) we 
sought to clarify the role of known adhesins in the BS induced increase in EHEC adhesion to epithelial cells.

Intimin is the best characterized adhesin and has been clearly demonstrated to play a key role in bacterial adhesion (Donnenberg et al., 1993; McKee et al., 1995; Torres et al., 2005b). Therefore, it is possible that intimin may play a role in increased adhesion after BS stress. To explore this hypothesis, an intimin mutant of 86-24 (8624 $e a e \Delta 10)$ and its complemented wild-type 86-24 eae $(\mathrm{pEB} 310)$ were used to investigate the adhesive capacity to human intestinal cells in tissue culture. First it was confirmed that the intimin negative mutant adhered much less than the parental strain or complemented intimin mutant, supporting the notion that intimin serves as a major adhesin (data not shown). We did note an adhesion enhancement of eae mutant after exposure to the BS mixture. The enhancement was 2.3 fold relative to that of the untreated correlate $(\mathrm{P}<0.05)$. Similar adhesion enhancement after treatment with the BS mixture was seen in 86-24 wt (2.1 fold increase) compared to the untreated control, which demonstrates that intimin is likely not the adhesin responsible for enhanced cell adhesion post-BS stress. Complementation of the intimin mutant with pEB310 restored adhesion to that of wt levels for both the unstressed and BS-stressed treatments. These results indicate that other colonization factors are involved in the increased adhesion to human intestinal cells after BS-treatment observed in vitro.

Since we concluded that intimin was not responsible for the increased bacterial adhesion observed after BS treatment, we choose to investigate another known bacterial adherence-conferring protein (Tha). This protein is another adhesin which has been described in E. coli $\mathrm{O} 157: \mathrm{H} 7$ to be involved in bacterial adhesion (Tarr et al., 2000).

To confirm that Iha is an adhesin and to exclude the effect of other adhesins, adherence to epithelial cells was assessed using iha positive strains (e.g. 86-24), an ihanegative mutant of 86-24 and a nonpathogenic E. coli strain (ORN172) that naturally lacks $i h a$, as well as this strain complemented with iha on a plasmid. Preliminary results showed that the 86-24( $\Delta i h a)$ mutant adhered significantly less $(\mathrm{P}<0.04)$ than the parental strain. This is consistent with previous reports, which indicated that Iha plays a role in adhesion of EHEC to host cells (Tarr et al., 2000). 
Additionally, an adhesion assay was performed for the ORN172(pSK + ) (plasmid alone, no iha) and ORN172(pIha) and results showed that E.coli ORN172(pIha) adhered $4 \mathrm{x}$ more than ORN172( $\mathrm{pSK}+$ ), confirming that iha plays a major role as an adhesion in a non-pathogenic $E$. coli background. The low levels of adherence in ORN172(pSK + ) can likely be attributed to type I fimbriae (Tarr et al., 2000). No significantly increased adherence was observed in either ORN172(pIha) or ORN172(pSK + ) after BS mix treatment as compared with untreated strains, suggesting that Iha is not responsible for the increase in adherence observed after BS stress, in these strains. This suggests that other adhesins or virulence factors that are not present in laboratory strains might be necessary to cause an increased adhesion after BS stress.

In order to confirm these results, protein expression under the same conditions needs to be examined. Further research is needed to fully explore the roles that these virulence factors have in host adhesion of BS stressed E. coli O157:H7 and their possible effects on Stx production.

\subsection{Short Chain Fatty Acid Stress Enhances Adhesion of E. coli 0157:H7 to Epithelial Cells}

In order to establish infection, $E$. coli $\mathrm{O} 157: \mathrm{H} 7$ must finally overcome the short chain fatty acid stress environment of the large intestine. SCFA represent approximately two-thirds of the colonic anion concentration (100-300 mM) mainly as acetate, propionate and butyrate (Cummings et al., 1987). It has been shown that gastrointestinal pathogens including EHEC possess sophisticated protective mechanisms to survive these conditions (Bearson et al., 1997; Kwon and Ricke, 1998; Durant et al., 1999; Ricke, 2003). Maintenance of this competitive edge is a prerequisite for efficient colonization and establishing infection of the large intestine by food-borne pathogens.

Significant research has been devoted to understanding the complex regulatory systems that mediate $E$. coli response to the external environment. However, there is very little information on the potential role of SCFA on virulence of gastrointestinal pathogens. To date several studies have demonstrated that bacteria exhibit enhanced virulence after SCFA stress. For example, Durant et al. (2000) showed that SCFA exposure increases the ability of stationary phase Salmonella typhimurium to adhere to 
HEp-2 cells. Recently another study by Rishi et al. (2005) reported that SCFA exposure of Salmonella enterica increased intracellular survival within macrophages, induced an acid tolerance response and enhanced expression of outer membrane matrix proteins (porins). According to Polen et al. (2003), E. coli growth in acetate and propionate showed enhanced flagellar motility. In contrast with previous studies, two other reports provided evidence for suppression of motility and chemotaxis at high $\mathrm{pH}$ (Polen et al., 2003). It is clear from these contradictory reports that the role of SCFA on virulence of E. coli $\mathrm{O} 157: \mathrm{H} 7$ in the host gastrointestinal tract is still currently unknown.

Since there are no previous studies that have addressed the role of SCFA on EHEC adherence capability, the present study aimed to clarify whether SCFA stress has any impact on EHEC adhesion to epithelial cells. Interestingly, the adhesion of SCFA stressed EHEC to HEp-2 cells was significantly higher compared to untreated EHEC ( $p<$ 0.05). Adhesion-enhancement factors ranged from 1.2 - 1.4, depending on SCFA treatment. We reasoned that the SCFA-induced adhesion of E. coli $\mathrm{O} 157: \mathrm{H} 7$ to host cells might be conferred by increased synthesis of existing or new adhesins. To determine whether the adhesion enhancement is a result of increased expression of adhesins, erythromycin, below the MIC, was used to block protein synthesis (de Jesus et al., 2005). Following SCFA-stress, all treatments were incubated with or without erythromycin and the adhesion of E. coli O157:H7 86-24 to HEp2 cells was examined. Pretreatment of SCFA stressed bacteria with erythromycin completely abolished the increased host adhesion capability of E. coli $\mathrm{O} 157: \mathrm{H} 7$ to Caco-2 cells compared to that of untreated sample. These results indicate that a specific set of proteins synthesized within $30 \mathrm{~min}$, after cells are exposed to SCFAs mixture, are responsible for the increased adhesion to host cells. Further studies are required to identify the nature of the adhesive factors involved in SCFA stressed increased adhesion.

\subsection{The Effect of Bile Salts and Short Chain Fatty Acids Treatments on $E$. coli O157:H7 Growth during Infection Time}

The enhanced adhesion to host epithelial cells by BS and SCFA stressed E. coli O157:H7 might be due to increased growth during infection time that may arise after BS 
or short chain fatty acid stress. To confirm this hypothesis, control adhesion assays were employed. E. coli O157:H7 86-24 was treated with either BS or SCFA and incubated without epithelial cells, simulating the adhesion assays previously described. Bacteria were then plated out and quantified. Three of the treatments showed significantly higher final viability compared to the control. These results indicate that the enhanced adhesion of BS stressed E. coli O157:H7 86-24 may be due in part to increased growth during infection time after stress. This is actually not a surprising observation since the viability of BS stressed EHEC with three of the treatments was increased relative to that of unshocked. On the other hand, unexpected results were obtained on EHEC growth during infection time after SCFA stress. Although there were no significant differences in initial amount of bacteria loaded, after $4 \mathrm{hr}$ incubation time the survival of all stressed bacteria was significantly lower in comparison to control. Therefore it is likely that enhanced adhesion after SCFA exposure is not mediated through increased growth during incubation time. Further studies will be needed to identify the requirements necessary for that butyrate and mixture adhesion enhancement.

\subsection{The Effect of Bile Salts and Short Chain Fatty Acids Treatment on E. coli O157:H7 86-24 Verotoxin Production:}

Stx production by EHEC 0157:H7 is the primary virulence factor associated with the development of the life threatening HUS and vascular complications of HC (Karmali et al., 1983). Karmaly et al., (1985) reported that verotoxin production is strain variable and classified Shiga-like toxin producing E.coli as low- to moderate-and high level toxin producers. The authors also suggested that Stx accumulated into the periplasmic space is released into the supernatant due to a leaky outer membrane (Karmali et al., 1985). Recently a study by House et al., (2006) examined the effect of acid shock of EHEC O157:H7 86-24 on verotoxin production and secretion. No significant difference was found in verotoxin secreted in the supernatant and that one retained in the periplasmic space, suggesting that acid shock does not augument the severity of EHEC induced renal disease.

Additional concern is that Shiga toxin has also been reported to enhance EHEC binding to epithelial cells which could lead to increased colonization, $\mathrm{HC}$ and its 
complications. One study by Robinson et al. (2006) reported that Stx2 produced during infection increases the cell surface expression of nucleolin and helps EHEC in promoting intestinal colonization. That Stx2 provides an adherence advantage of EHEC 0157:H7 in establishing infection was supported by the finding that wt EHEC 86-24 adhere better to epithelial cells than the $s t x 2$ isogenic mutant (Robinson et al., 2006). Epithelial cells that were exposed to enzymatically active Stx2 toxin throughout the infection period not only harmed the host but also enhanced the bacterial adherence level of stx 2 isogenic mutants. This increased adherence of isogenic mutants correlated with toxin exposure was even higher to HEp-2 cells than its wt 86-24 alone. These findings are in agreement with their previous report that demonstrated that intimin interacts not only with Tir as a receptor but also with the eukaryotic protein nucleolin localized on the cell surface (Sinclair \& O'Brien, 2002). Similar results were obtained by Barnett Foster et al, (2000) that demonstrated an enhanced adhesion of EHEC to Stx-treated epithelial cells. This finding was correlated with increased levels of outer leaflet phosphatidyletanolamine, the host receptor previously reported to bind EHEC (Barnett Foster et al., 2000).

This study investigated the impact of BS and SCFA treatment on both secreted and periplasmic production of verotoxin. It is important to assay the levels of both secreted and periplasmic production because it is well documented that antimicrobial treatment of EHEC infections can activate the Stx phage thereby releasing the toxin accumulated in the periplasmic space and increasing the risk of HUS (Bushen and Guerrant, 2003; Kaper et al., 2004).

\subsubsection{No Change in Secreted and Periplasmic VT Production after Bile Salt Treatment}

Firstly, the impact of BS stress on Stx production (both secreted and periplasmic extracts) of E. coli O157:H7 86-24 was examined. The results of the Vero cell cytotoxicity assay showed no significant changes in toxin production either in the supernatant filtrates or periplasmic extract across all BS various treatments. Since no significant differences were found across all the various treatments, it is likely that BS stress does not augment the severity of EHEC induced renal disease. 


\subsubsection{Increased VT Levels after Short Chain Fatty Acids Mixture Stress}

Having shown the impact of BS on Stx production, further investigation was conducted to determine whether the Stx levels were altered by SCFA stress. SCFAmixture treatment resulted in a significant increase in Stx production in both supernatant filtrates and periplasmic extracts compared to untreated controls. In contrast, acetate induced Stx production was significantly lower compared to untreated controls. Butyrate and propionate treatments did not affect the Stx production, either secreted or periplasmic. These data suggest that exposure to an SFCA mixture almost similar to that of the large intestine increases Stx production and extrapolated to the in vivo situation, may augment the severity of EHEC induced renal disease by increasing levels of Stx produced. This is consistent with the proposed mechanism in which environmental signals in the colon serve as cue for bacterial infection (Gyles et al., 2006). Research by Louise et al. (1995) showed that butyrate increases the number of Stx receptors on HUVEC cell surface. Although it is tempting to suggest that butyrate may increase Stx binding to $\mathrm{Gb3}$, further study is needed. However, it is possible that $E$ coli $\mathrm{O} 157: \mathrm{H} 7$ exploits the SCFA microenvironment using it as a signal to seek appropriate opportunities for infection in the large intestine. Further research is required to examine whether other E. coli $\mathrm{O} 157: \mathrm{H} 7$ strains produce the same observed results observed here under these experimental conditions. A better understanding will be obtained by analyzing the molecular basis of these changes in gene expression induced by BS and SCFA treatments of E. coli $\mathrm{O} 157: \mathrm{H} 7$. 


\section{Summary and Conclusions}

\subsection{Summary of Results}

This research examines the impact of exposure of EHEC O157:H7 to physiologically relevant concentrations of BS and SCFA on critical virulence properties including the adhesion to host endothelial cells and the production of Stxs. This study has revealed significant stress-induced changes in a number of these properties including survival, ability to adhere to human epithelial cells and verotoxin production. Exposure to BS significantly enhanced the survival of EHEC O157:H7. It is tempting to speculate that this increase in survival first could facilitate the recovery from the acid stress of the stomach and further promote colonization in the large intestine. There was also significantly increased adhesion to tissue culture cells after BS stress. Our investigations on EHEC adherence capability after BS treatment were extended for two different epithelial cell lines and we found that EHEC had the highest percent of viable bacteria bound to Caco- 2 cells ( $4 x$ fold increase) and the lowest adherence level ( $2 x$ fold increase) to HEp-2 cells.

Another interesting finding was that the pretreatment of the bacteria with erythromycin completely abolished the adhesion enhancement of BS stressed EHEC O157:H7. This suggests that the enhanced adhesion required increased bacterial protein synthesis, presumably of existing or new adhesins. The adhesion increase after BS exposure was likely not mediated by either an intimin or Tha since the same adhesion increase was noted for both the intimin-negative mutant and the Iha negative mutant. On the other hand, a nonpathogenic E. coli strain demonstrated decreased adhesion after BS stress. Adhesion levels of both BS treated and untreated nonpathogenic ORN172 strains were much lower relative to E. coli $\mathrm{O} 157: \mathrm{H} 7$ strain. No variation of the host adhesion capability of non-virulent laboratory K12 strains to HEp-2 cells indicate that virulence factors not present in the laboratory strains are responsible for an increased level of adhesion to host cells in EHEC.

Next, we hypothesized that the enhanced adhesion might be due to increased growth after BS stress. Preliminary data demonstrated that glycocholate, deoxycholate 
and ursocholate treatments showed significantly higher final viability compared to the control. It is likely possible that the glycocholate treatment may have enhanced the adhesion of E. coli $\mathrm{O} 157: \mathrm{H} 7$ to epithelial cells through elevated growth after stress. This would suggest that there are more bacteria adhering because there are more bacteria present. These findings are consistent with previous studies in the lab that have also demonstrated that BS treatments significantly enhanced several other virulence properties including motility, flagella expression and resistance to several antibiotics (Prayitno et al., unpublished data). None of the experimental BS treatments resulted in a significant change in Stx production in either secreted or periplasmic extracts. These results suggest that BS does not upregulate STx production and so this virulence factor is not expressed at higher levels after BS stress. This could then be used to suggest that BS stress may not lead to increased risk of VT -mediated pathology includind HUS. However, further research needs to clarify wheter more VT is produced that more is translocated into the blood stream and that more reaches the kidneys etc and causes damage. Since VT has also been implicated in increased adhesion of the bacteria, it is unlikely that changes in VT levels could explain the increased adhesion observed for BS-stressed EHEC. It is tempting to speculate that EHEC sense the BS as a signal of being in the small intestine and does not influence the Stx production in order to save resources and promote colonization in more appropriate environments such as the large intestine.

Since BS stress may be inducing novel adhesin expression, microarray analysis is currently underway (Barnett Foster, D.E., personal communication). Any potential adhesins upregulated will be confirmed with real time RT-PCR and BS adhesion assays.

Having shown the impact of BS on adherence capability and verotoxin production levels, further investigation was conducted to determine whether SCFA stress had any effect on the adhesion and cytotoxicity. SCFA stress, such as that present in the large intestine, significantly induces changes in bacterial survival, ability to adhere to human epithelial cells and verotoxin production. Interestingly, although the survival after exposure to SCFAs was significantly lower than that of untreated control, it seems that bacteria that survived the stress showed enhanced adhesion capabilities to host epithelial cells. Pretreatment of SCFA stressed E.coli O157:H7 strains with subinhibitory concentrations of erythromycin did not show increased adhesion to host epithelial cells. 
These data again support the idea that protein synthesis was required for the increased adhesion phenotype and strongly suggest that the adhesion enhancement resulted from increased expression of existing or new adhesins.

Treatment with the SCFA-mixture resulted in significantly increased Stx production in both supernatant filtrates and periplasmic extracts compared to untreated controls. This finding lends support of the concept that EHEC is using local microenvironmental cues to regulate virulence factor expression. The mix of SFCAs approximates that of the local microenvironment of the large intestine and the data suggest that EHEC is responding to this cue by increasing the production of VT in a location where this virulence factor can be translocated across the intestinal epithelial for circulation to body organs including kidneys. Since the bacterial survival is lower after SCFA exposure, the data might also suggest that increased Stx production may assist in enhancing bacterial adhesion. Further work is needed to determine whether Stx mediated adherence contributes to and consolidates the SCFA induced changes. Again, these findings are consistent with previous studies in the lab that have also proven that SCFA treatments significantly enhanced other several virulence properties including flagella expression (Prayitno et al., unpublished data).

\subsection{Future Work}

Since the results of this study highlighted changes in virulence factors, the molecular basis of the response of EHEC to localized stress in the small and large intestine should be examined. We hypothesize that the changes in expression of selected virulence factors is a direct response to the localized environmental stress encountered during transition to the small and large intestine. Whole-genome expression profiling (microarray analysis) and real-time PCR are currently underway to examine changes in the expression of genes up- and down-regulated post BS- and SCFA- treatment. This research should begin by focusing on genes encoding adhesin candidates since the most recent data from this study indicate significant changes in host adhesion after specific SCFA stress. Based on these results, the role of these candidate genes in host cell 
infection should be assessed by constructing mutants defective in the individual genes and then should be tested in phenotypic virulence assays.

This study has highlighted changes in several virulence factors including adhesion to host tissues; the role(s) of known bacterial adhesins after BS and SCFA stress needs to be clarified. BS and SCFA adhesion assays with espA, iha and intimin mutants should be completed. Furthermore, Western blot analysis should be employed to assess expression levels of intimin, Iha and EspA in BS and SCFA-treated organisms. Additionally, the secretion phenotypes of BS and SCFA stressed E. coli O157:H7 should also be examined to further explore the possible posttranscriptional regulation of these virulence factors after either BS or SCFA treatments. The role of other potential adhesins, such as OmpA, EspA, long polar fimbriae 2, and Efa-1 should also be examined after either BS or SCFA stress. Adhesion assays with parental and mutant strains for each adhesin should be conducted. Microscopic investigation would also be useful to determine if there are differences between stressed and unstressed strains. This research will enhance our understanding of stress-induced virulence changes in this serious, potentially lifethreatening gastrointestinal pathogen and explain the genetic basis of these changes.

\subsection{Significance and Conclusions}

It is obvious that most bacterial pathogens including E. coli $0157: \mathrm{H} 7$ must overcome hostile conditions, such those encountered in the intestinal tract, in order to successfully reach their target sites on the surface of enterocytes (Merrell and Camilli, 2002; Foster, 2004). The results of this study show that the environmental conditions of the human host, such as BS of the small intestine and SCFA of the large intestine, directly influence essential virulence factors involved in the intestinal colonization process of E. coli $\mathrm{O} 157: \mathrm{H} 7$ including bacterial attachment to epithelial cells as well as verotoxin production. Interestingly, this work suggests for the first time that $E$. coli O157:H7 uses BS and SCFA as a cue to establish location and influence virulence gene expression. The production of verotoxin is the major virulence factor in EHEC infection since it can ultimately lead to several life threatening diseases such as hemolytic uremic syndrome and thrombic thrombocytopenic purpoura. SCFA stress did significantly increased verotoxin production. This finding is significant and supports the idea that 
exposure to SCFA stress will lead to increased cases of HUS due to increased verotoxin production. Adhesion to host cells is an important virulence factor of EHEC infection. Although not associated with the severe life threatening disease, adherence to host epithelial cells is still a requirement for the development of diarrhea (Tzipori et al., 1987; Campellone and Leong, 2003). Since BS treatment has enhanced survival and adhesion capability to the host cells, the severity of EHEC-induced diarrhea may be increased and may occur faster in infected individuals. Taken together, these results indicate that $E$. coli is able to survive in hostile conditions, reproducing stresses encountered in the intestinal tract, a prerequisite for successful propagation of the microorganisms. However, the higher levels of adhesion to intestinal cells observed with SCFA stress could be attributed to an advantage due to the increased verotoxin production. The results of this research indicate that ingestion stresses such as BS and SCFA, which are part of the host's natural chemical assault on foreign organisms, may actually enhance the virulence properties of this pathogen including adhesion to human epithelial cells, Stx production, motility and flagella expression, resistance to several antibiotics contributing to, rather than, preventing infection. Furthermore, they suggest that this pathogen may use these ingestion stresses to cue the expression of numerous virulence factors for successful infection of local microenvironments.

This work makes a valuable contribution to understanding how these stresses modulate the virulence potential of this pathogen. In addition, we have contributed essential data for delineating the pathogenesis of disease caused by EHEC infection. Continued work in this field will surely contribute to identifying new genes and pathways involved in BS and SCFA stress-induced virulence changes. These data, combined with other results, may provide exciting possible alternatives for the treatment or prevention of EHEC infections. It may allow the design of a new class of antimicrobials that can block BS and SCFA signaling in bacterial pathogens. These potential antimicrobials will not only be useful against EHEC but possibly also against other pathogens which harbor similar response to stress. We can conclude that our hypothesis was correct. Finally, our results support the hypothesis that environmental stresses such as BS or SCFA encountered during transition to the small and large intestine increase EHEC virulence including host cell adhesion and toxin production. 


\section{Supplementary Data}

\section{Supplemental Table 1}

Summary of Adhesion of E. coli strains to HEp-2/Caco-2 cells before and after BS Exposure as Determined by Plate-count assay

Table 6-1 Supplemental Data 1

\begin{tabular}{|c|c|c|c|c|c|}
\hline \multirow[t]{2}{*}{ Strain } & \multirow[t]{2}{*}{ Treatment } & \multicolumn{2}{|c|}{ HEp-2 } & \multicolumn{2}{|c|}{ Caco-2 } \\
\hline & & $\%$ & $\begin{array}{c}\text { Fold } \\
\text { increase }\end{array}$ & $\%$ & $\begin{array}{c}\text { Fold } \\
\text { increase }\end{array}$ \\
\hline $86-24$ & UU & $2.5-2.73 \%$ & & $4.37 \%$ & \\
\hline $86-24$ & Gly & $4.36 \%$ & $1.5 x$ & $4.87 \%$ & $1.1 x$ \\
\hline $86-24$ & Mix & $4.05-5.29 \%$ & $1.5-2.1 x$ & $20.91 \%$ & $4.7 x$ \\
\hline $86-24 e a e \Delta 10$ & UU & $0.3-0.34 \%$ & & & \\
\hline $86-24 e a e \Delta 10$ & Mix & $0.70 \%$ & $2.3 x$ & & \\
\hline 86-24eae $\Delta(\mathrm{pEB} 310)$ & UU & $4.70 \%$ & & & \\
\hline $86-24 e a e \Delta(\mathrm{pEB} 310)$ & Mix & $6.74 \%$ & $1.4 x$ & & \\
\hline $86-24 \Delta i h a$ & UU & $1.69 \%$ & & & \\
\hline 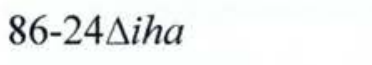 & Mix & $2.88 \%$ & $1.7 x$ & & \\
\hline ORN172(pIHA) & UU & $1.69 \%$ & & & \\
\hline ORN172(pIHA) & Mix & $2.16 \%$ & & & \\
\hline ORN172(pSK +$)$ & UU & $0.59 \%$ & & & \\
\hline ORN172(pSK+) & $\operatorname{Mix}$ & $0.92 \%$ & & & \\
\hline
\end{tabular}

Note: Adhesion enhancement factors after BS exposure ranged from 1.1 -4.7x depending on the strain used and treatments. 


\section{Supplemental Table 2}

Summary of Adhesion of E. coli strains to HEp-2/Caco-2 cells before and after SCFA Exposure as Determined by Plate-count assay

Table 6-2 Supplemental Data 2

\begin{tabular}{rr|rrrr}
\hline \multirow{2}{*}{ Strain } & \multirow{2}{*}{ Treatment } & \multicolumn{3}{|c}{ HEp-2 } & \multicolumn{2}{c}{ Caco-2 } \\
& & \multicolumn{1}{|c}{$\%$} & Fold increase & \multicolumn{1}{c}{$\%$} & Fold increase \\
\cline { 3 - 6 } & & & & & \\
$86-24$ & UU & $15-20 \%$ & & $25.00 \%$ & \\
$86-24$ & But & $28-30 \%$ & $\mathbf{1 . 5}-\mathbf{1 . 8 x}$ & $28.00 \%$ & $\mathbf{1 . 1 x}$ \\
$86-24$ & Mix & $25-60 \%$ & $\mathbf{1 . 6}-\mathbf{3 x}$ & $50.00 \%$ & $\mathbf{2 x}$ \\
\hline
\end{tabular}

Note: Adhesion enhancement factors after SCFA exposure ranged from 1.1 -3x depending on the strain used and treatments 
Abdul-Raouf, U. M., Beuchat, L. R., \& Ammar, M. S. (1993). Survival and growth of Escherichia coli O157:H7 on salad vegetables. Appl. Environ. Microbiol., 59(7), 1999-2006.

Abe, H., Tatsuno, I., Tobe, T., Okutani, A., \& Sasakawa, C. (2002). Bicarbonate Ion Stimulates the Expression of Locus of Enterocyte Effacement-Encoded Genes in Enterohemorrhagic Escherichia coli 0157:H7. Infection and Immunity, 70(7), 3500-3509.

Adams, T. M., Wentzel, A., \& Kolmar, H. (2005). Intimin-Mediated Export of Passenger Proteins Requires Maintenance of a Translocation-Competent Conformation. Jurnal of Bacteriology, 187(2), 522-533

Allerberger, F., \& Guggenbichler, J. P. (1989). Listeriosis in Austria--report of an outbreak in 1986. Acta microbiologica Hungarica, 36(2-3), 149-152.

Arnold, C. N., McElhanon, J., Lee, A., Leonhart, R., \& Siegele, D. A. (2001). Global Analysis of Escherichia coli Gene Expression during the Acetate-Induced Acid Tolerance Response. J. Bacteriol., 183(7), 2178-2186.

Badea, L., Doughty, S., Nicholls, L., Sloan, J., Robins-Browne, R. M., \& Hartland, E. L. (2003). Contribution of Efa1/LifA to the adherence of enteropathogenic Escherichia coli to epithelial cells (Vol. 34): Elsevier Science.

Barnett Foster, D., Abul-Milh, M., Huesca, M., \& Lingwood, C. A. (2000). Enterohemorrhagic Escherichia coli Induces Apoptosis Which Augments Bacterial Binding and Phosphatidylethanolamine Exposure on the Plasma Membrane Outer Leaflet. Infect. Immun., 68(6), 3108-3115.

Bearson, S., Bearson, B., \& Foster, J. W. (1997). Acid stress responses in enterobacteria. FEMS Microbiology Letters, 147(2), 173-180.

Begley, M., Gahan, C. G. M., \& Hill, C. (2002). Bile Stress Response in Listeria monocytogenes LO28: Adaptation, Cross-Protection, and Identification of Genetic Loci Involved in Bile Resistance. Appl. Environ. Microbiol., 68(12), 6005-6012.

Begley, M., Gahan, C. G. M., \& Hill, C. (2005). The interaction between bacteria and bile. FEMS Microbiology Reviews, 29(4), 625-651.

Bernstein, C., Bernstein, H., Payne, C. M., Beard, S. E., \& Schneider, J. (1999). Bile Salt Activation of Stress Response Promoters in Escherichia coli. Current Microbiology, 39(2), 68-72.

Berryman, M., Franck, Z., \& Bretscher, A. (1993). Ezrin is concentrated in the apical microvilli of a wide variety of epithelial cells whereas moesin is found primarily in endothelial cells. J Cell Sci, 105(4), 1025-1043. 
Boerlin, P., McEwen, S. A., Boerlin-Petzold, F., Wilson, J. B., Johnson, R. P., \& Gyles, C. L. (1999). Associations between Virulence Factors of Shiga Toxin-Producing Escherichia coli and Disease in Humans. J. Clin. Microbiol., 37(3), 497-503.

Borczyk, A. A., Karmali, M. A., Lior, H., \& Duncan, L. M. C. (1987). Bovine Reservoir for verotoxin-producing Escherichia coli O157:H7. The Lancet, 329(8524), 9898.

Borthakur, A., Gill, R. K., Hodges, K., Ramaswamy, K., Hecht, G., \& Dudeja, P. K. (2006). Enteropathogenic Escherichia coli inhibits butyrate uptake in Caco-2 cells by altering the apical membrane MCT1 level. Am J Physiol Gastrointest Liver Physiol, 290(1), G30-35.

Briones, V., Blanco, M. M., Marco, A., Prats, N., Fernández-Garayzábal, J. F., Suárez, G., Domingo, M., \& Domínguez, L. (1992). Biliary excretion as possible origin of Listeria monocytogenes in fecal carriers. American journal of veterinary research, 53(2), 191-193.

Brook, I. (1989). Aerobic and anaerobic microbiology of biliary tract disease. J. Clin. Microbiol., 27(10), 2373-2375.

Brunder, W., Khan, A. S., Hacker, J., \& Karch, H. (2001). Novel Type of Fimbriae Encoded by the Large Plasmid of Sorbitol-Fermenting Enterohemorrhagic Escherichia coli O157:H-. Infect. Immun., 69(7), 4447-4457.

Bushen, O. Y., \& Guerrant, R. L. (2003). Acute Infectious Diarrhea. Topics in Emergency Medicine, 25(2), 139.

Bustamante, V. H., Santana, F. J., Calva, E., \& Puente, J. L. (2001). Transcriptional regulation of type III secretion genes in enteropathogenic Escherichia coli: Ler antagonizes H-NS-dependent repression. Molecular Microbiology, 39(3), 664678.

Cable, C. S., Rebhun, W. C., \& Fortier, L. A. (1997). Cholelithiasis and cholecystitis in a dairy cow. Journal of the American Veterinary Medical Association, 211(17), 899-900.

Campellone, K., Robbins, D., \& Leong, J. (2004). EspF(U) is a translocated EHEC effector that interacts with Tir and N-WASP and promotes Nckindependent actin assembly. Developmentall Cell, 7(2), 217-228.

Campellone, K. G., \& Leong, J. M. (2003). Tails of two Tirs: actin pedestal formation by enteropathogenic E. coli and enterohemorrhagic E. coli O157:H7. Current Opinion in Microbiology, 6(1), 82-90. 
Cantarelli, V. V., Takahashi, A., Yanagihara, I., Akeda, Y., Imura, K., Kodama, T., Kono, G., Sato, Y., Iida, T., \& Honda, T. (2002). Cortactin Is Necessary for FActin Accumulation in Pedestal Structures Induced by Enteropathogenic Escherichia coli Infection. Infect. Immun., 70(4), 2206-2209.

Caprioli, A., Morabito, S., Brugère, H., \& Oswald, E. (2005). Enterohaemorrhagic Escherichia coli: emerging issues on virulence and modes of transmission. Veterinary Research, 36(3), 289-311.

Carey, C. M., Kostrzynska, M., Ojha, S., \& Thompson, S. (2008). The effect of probiotics and organic acids on Shiga-toxin 2 gene expression in enterohemorrhagic Escherichia coli 0157:H7 Journal of Microbiological Methods, 73(2), 125-132.

Carlier, M. F., Wiesner, S., Le Clainche, C., \& Pantaloni, D. (2003). Actin-based motility as a self-organized system: mechanism and reconstitution in vitro. Comptes Rendus Biologies, 326, 161-170.

Carpenter, H. A. (1998). Bacterial and Parasitic Cholangitis. Mayo Clin Proc, 73(5), 473478.

Ceponis, P. J. M., McKay, D. M., Ching, J. C. Y., Pereira, P., \& Sherman, P. M. (2003). Enterohemorrhagic Escherichia coli O157:H7 Disrupts Stat1-Mediated Gamma Interferon Signal Transduction in Epithelial Cells. Infect. Immun., 71(3), 13961404.

Ceponis, P. J. M., Riff, J. D., \& Sherman, P. M. (2005). Epithelial cell signaling responses to enterohemorrhagic Escherichia coli infection. Memórias do Instituto Oswaldo Cruz, 100, 199-203.

Cheville, A. M., Arnold, K. W., Buchrieser, C., Cheng, C. M., \& Kaspar, C. W. (1996). rpoS regulation of acid, heat, and salt tolerance in Escherichia coli O157:H7. Appl. Environ. Microbiol., 62(5), 1822-1824.

Chou, C.-C., \& Cheng, S.-J. (2000). Recovery of low-temperature stressed E. coli O157:H7 and its susceptibility to crystal violet, bile salt, sodium chloride and ethanol. International Journal of Food Microbiology, 61(2-3), 127-136.

Clarke, M. B., \& Sperandio, V. (2005). Events at the Host-Microbial Interface of the Gastrointestinal Tract III. Cell-to-cell signaling among microbial flora, host, and pathogens: there is a whole lot of talking going on. Am J Physiol Gastrointest Liver Physiol, 288(6), G1105-1109.

Coleman, R., Lowe, P. J., \& Billington, D. (1980). Membrane lipid composition and susceptibility to bile salt damage. Biochimica et Biophysica Acta, 599(1), 294300 . 
Crane, J. K., Majumdar, S., \& Pickhardt, D. F., III. (1999). Host Cell Death due to Enteropathogenic Escherichia coli Has Features of Apoptosis. Infect. Immun., 67(5), 2575-2584.

Cummings, J. H., Pomare, E. W., Branch, W. J., Naylor, C. P., \& Macfarlane, G. T. (1987). Short chain fatty acids in human large intestine, portal, hepatic and venous blood. Gut, 28(10), 1221-1227.

Dahan, S., Knutton, S., Shaw, R. K., Crepin, V. F., Dougan, G., \& Frankel, G. (2004). Transcriptome of Enterohemorrhagic Escherichia coli 0157 Adhering to Eukaryotic Plasma Membranes. Infect. Immun., 72(9), 5452-5459.

de Jesus, M. C., Urban, A. A., Marasigan, M. E., \& Foster, D. E. B. (2005). Acid and Bile-Salt Stress of Enteropathogenic Escherichia coli Enhances Adhesion to Epithelial Cells and Alters Glycolipid Receptor Binding Specificity. Journal of Infectious Diseases, 192(8), 1430-1440.

De Wulf, P., McGuire, A. M., Liu, X., \& Lin, E. C. C. (2002). Genome-wide Profiling of Promoter Recognition by the Two-component Response Regulator CpxR-P in Escherichia coli. J. Biol. Chem., 277(29), 26652-26661.

Delahay, R. M., Knutton, S., Shaw, R. K., Hartland, E. L., Pallen, M. J., \& Frankel, G. (1999). The Coiled-coil Domain of EspA Is Essential for the Assembly of the Type III Secretion Translocon on the Surface of Enteropathogenic Escherichia coli. J. Biol. Chem., 274(50), 35969-35974.

Deng, W., Li, Y., Hardwidge, P. R., Frey, E. A., Pfuetzner, R. A., Lee, S., Gruenheid, S., Strynakda, N. C. J., Puente, J. L., \& Finlay, B. B. (2005). Regulation of Type III Secretion Hierarchy of Translocators and Effectors in Attaching and Effacing Bacterial Pathogens. Infect. Immun., 73(4), 2135-2146.

Deng, W., Puente, J. L., Gruenheid, S., Li, Y., Vallance, B. A., Vázquez, A., Barba, J., Ibarra, J. A., O'Donnell, P., Metalnikov, P., Ashman, K., Lee, S., Goode, D., Pawson, T., \& Finlay, B. B. (2004). Dissecting virulence: Systematic and functional analyses of a pathogenicity island. Proceedings of the National Academy of Sciences of the United States of America, 101(10), 3597-3602.

DiGiuseppe, P. A., \& Silhavy, T. J. (2003). Signal Detection and Target Gene Induction by the CpxRA Two-Component System. J. Bacteriol., 185(8), 2432-2440.

Donnenberg, M. S., Tzipori, S., McKee, M. L., O'Brien, A. D., Alroy, J., \& Kaper, J. B. (1993). The role of the eae gene of enterohemorrhagic Escherichia coli in intimate attachment in vitro and in a porcine model. The Journal of Clinical Investigation, 92(3), 1418-1424. 
Durant, J. A., Corrier, D. E., Byrd, J. A., Stanker, L. H., \& Ricke, S. C. (1999). Feed Deprivation Affects Crop Environment and Modulates Salmonella enteritidis Colonization and Invasion of Leghorn Hens. Appl. Environ. Microbiol., 65(5), 1919-1923.

Dytoc, M., Soni, R., Cockerill, F., 3rd, De Azavedo, J., Louie, M., Brunton, J., \& Sherman, P. (1993). Multiple determinants of verotoxin-producing Escherichia coli O157:H7 attachment-effacement. Infect. Immun., 61(8), 3382-3391.

Dziva, F., van Diemen, P. M., Stevens, M. P., Smith, A. J., \& Wallis, T. S. (2004). Identification of Escherichia coli $\mathrm{O} 157$ : $\mathrm{H} 7$ genes influencing colonization of the bovine gastrointestinal tract using signature-tagged mutagenesis. Microbiology, 150(11), 3631-3645.

Elhanafi, D., Leenanon, B., Bang, W., \& Drake, M. A. (2004). Impact of Cold and ColdAcid Stress on Poststress Tolerance and Virulence Factor Expression of Escherichia coli O157:H7. Journal of Food Protection, 67, 19-26.

Ferreira, A., O'Byrne, C. P., \& Boor, K. J. (2001). Role of \{sigma\}B in Heat, Ethanol, Acid, and Oxidative Stress Resistance and during Carbon Starvation in Listeria monocytogenes. Appl. Environ. Microbiol., 67(10), 4454-4457.

Flores, C., Maguilnik, I., Hadlich, E., \& Goldani, L. Z. (2003). Microbiology of choledochal bile in patients with choledocholithiasis admitted to a tertiary hospital. Journal of Gastroenterology \& Hepatology, 18(3), 333-336.

Foster, J. (2004). Escherichia coli acid resistance: tales of an amateur acidophile. Nature Reviews Microbiology, 2(11), 898-907.

Fox, J. G., Yan, L. L., Dewhirst, F. E., Paster, B. J., Shames, B., Murphy, J. C., Hayward, A., Belcher, J. C., \& Mendes, E. N. (1995). Helicobacter bilis sp. nov., a novel Helicobacter species isolated from bile, livers, and intestines of aged, inbred mice. J. Clin. Microbiol., 33(2), 445-454.

Fraser, M. E., Fujinaga, M., Cherney, M. M., Melton-Celsa, A. R., Twiddy, E. M., O'Brien, A. D., \& James, M. N. G. (2004). Structure of Shiga Toxin Type 2 (Stx2) from Escherichia coli O157:H7. J. Biol. Chem., 279(26), 27511-27517.

Gabig, M., Herman-Antosiewicz, A., Kwiatkowska, M., Los, M., Thomas, M. S., \& Wegrzyn, G. (2002). The cell surface protein Ag43 facilitates phage infection of Escherichia coli in the presence of bile salts and carbohydrates. Microbiology, 148(5), 1533-1542.

Gansheroff, L. J., Wachtel, M. R., \& O'Brien, A. D. (1999). Decreased Adherence of Enterohemorrhagic Escherichia coli to HEp-2 Cells in the Presence of Antibodies 
That Recognize the C-Terminal Region of Intimin. Infect. Immun., 67(12), 64096417.

Gantois, I., Ducatelle, R., Pasmans, F., Haesebrouck, F., Hautefort, I., Thompson, A., Hinton, J. C., \& Van Immerseel, F. (2006). Butyrate Specifically Down-Regulates Salmonella Pathogenicity Island 1 Gene Expression. Appl. Environ. Microbiol., 72(1), 946-949.

Gänzle, M. G., Hertel, C., van der Vossen, J. M. B. M., \& Hammes, W. P. (1999). Effect of bacteriocin-producing lactobacilli on the survival of Escherichia coli and Listeria in a dynamic model of the stomach and the small intestine. International Journal of Food Microbiology, 48(1), 21-35.

Garmendia, J., Frankel, G., \& Crepin, V. F. (2005). Enteropathogenic and Enterohemorrhagic Escherichia coli Infections: Translocation, Translocation, Translocation. Infect. Immun., 73(5), 2573-2585.

Gauthier, A., Puente, J. L., \& Finlay, B. B. (2003). Secretin of the Enteropathogenic Escherichia coli Type III Secretion System Requires Components of the Type III Apparatus for Assembly and Localization. Infect. Immun., 71(6), 3310-3319.

Gobert, A. P., Vareille, M., Glasser, A.-L., Hindre, T., de Sablet, T., \& Martin, C. (2007). Shiga Toxin Produced by Enterohemorrhagic Escherichia coli Inhibits PI3K/NF\{kappa\}B Signaling Pathway in Globotriaosylceramide-3-Negative Human Intestinal Epithelial Cells. J Immunol, 178(12), 8168-8174.

Goldberg, M. D., Johnson, M., Hinton, J. C. D., \& Williams, P. H. (2001). Role of the nucleoid-associated protein Fis in the regulation of virulence properties of enteropathogenic Escherichia coli. Molecular Microbiology, 41(3), 549-559.

Grant, A. J., Farris, M., Alefounder, P., Williams, P. H., Woodward, M. J., \& O'Connor, C. D. (2003). Co-ordination of pathogenicity island expression by the BipA GTPase in enteropathogenic Escherichia coli (EPEC). Molecular Microbiology, $48(2), 507-521$.

Grys, T. E., Siegel, M. B., Lathem, W. W., \& Welch, R. A. (2005). The StcE Protease Contributes to Intimate Adherence of Enterohemorrhagic Escherichia coli O157:H7 to Host Cells. Infect. Immun., 73(3), 1295-1303.

Gunn, J. S. (2000). Mechanisms of bacterial resistance and response to bile. Microbes and Infection, 2(8), 907-913.

Gupta, S., \& Chowdhury, R. (1997). Bile affects production of virulence factors and motility of Vibrio cholerae Infection and Immunity, 65(3), 1131-1134. 
Hacker, J., \& Kaper, J. B. (2000). Pathogenicity islands and the evolution of microbes. Annual Review of Microbiology, 54(1), 641-679.

Hardwidge, P. R., Rodriguez-Escudero, I., Goode, D., Donohoe, S., Eng, J., Goodlett, D. R., Aebersold, R., \& Finlay, B. B. (2004). Proteomic Analysis of the Intestinal Epithelial Cell Response to Enteropathogenic Escherichia coli. J. Biol. Chem., 279(19), 20127-20136.

Hayashi, T., Makino, K., Ohnishi, M., Kurokawa, K., Ishii, K., Yokoyama, K., Han, C.G., Nakayama, K., Murata, T., Tanaka, M., Tobe, T., Iida, T., Honda, T., Takami, H., sasakawa, C., Ogasawara, N., Yasunaga, T., Kuhara, S., Shiba, T., Hattori, M., \& Shinagawa, H. (2001). Complete Genome Sequence of Enterohemorrhagic Eschelichia coli O157:H7 and Genomic Comparison with a Laboratory Strain K12 (Supplement). DNA Res, 8(1), 47-52.

Hayward, R. D., Leong, J. M., Koronakis, V., \& Campellone, K. G. (2006). Box 1 | Pathogenic Escherichia coli deliver multiple effector proteins into mammalian cells. Nature Reviews Microbiology, 4, 358-370.

Hecht, G., \& Koutsouris, A. (1999). Enteropathogenic E. coli attenuates secretagogueinduced net intestinal ion transport but not $\mathrm{Cl}$ - secretion. Am $J$ Physiol Gastrointest Liver Physiol, 276(3), G781-788.

Herold, S., Karch, H., \& Schmidt, H. (2004). Shiga toxin-encoding bacteriophages genomes in motion (Vol. 294): Elsevier Science.

Hoey, D. E. E., Sharp, L., Currie, C., Lingwood, C. A., Gally, D. L., \& Smith, D. G. E. (2003). Verotoxin 1 binding to intestinal crypt epithelial cells results in localization to lysosomes and abrogation of toxicity. Cellular Microbiology, 5(2), 85-97.

Hofmann, A. F. (1999). Bile Acids: The Good, the Bad, and the Ugly. News Physiol Sci, 14(1), 24-29.

House, B., Mair, R., Cvitkovitch, D., Gannon, V., \& Foster Barnett, D. (2006). StressInduced Changes in Enterohemorrhagic Escherichia coli O157 Virulence. Sixth International. Paper presented at the Symposium on Shiga Toxin (Verocytotoxin) Producing E. coli Infections (VTEC 2006).

Huesca, M., Borgia, S., Hoffman, P., \& Lingwood, C. A. (1996). Acidic pH changes receptor binding specificity of Helicobacter pylori: a binary adhesion model in which surface heat shock (stress) proteins mediate sulfatide recognition in gastric colonization. Infect. Immun., 64(7), 2643-2648.

Hughes, D. T., \& Sperandio, V. (2008). Inter-kingdom signalling: communication between bacteria and their hosts. Nat Rev Micro, 6(2), 111-120. 
Hung, D. T., Zhu, J., Sturtevant, D., \& Mekalanos, J. J. (2006). Bile acids stimulate biofilm formation in Vibrio cholerae. Molecular Microbiology, 59, 193-201.

Inan, M. S., Rasoulpour, R. J., Yin, L., Hubbardd, A. K., Rosenberg, D. W., \& Giardina, C. (2000). The luminal short-chain fatty acid butyrate modulates NF- $\hat{I}^{\circ} \mathrm{B}$ activity in a human colonic epithelial cell line. Gastroenterology, 118(4), 724-734.

Jerse, A. E., \& Kaper, J. B. (1991). The eae gene of enteropathogenic Escherichia coli encodes a 94-kilodalton membrane protein, the expression of which is influenced by the EAF plasmid. Infect. Immun., 59(12), 4302-4309.

Kaper, J. B., Gansheroff, L. J., Wachtel, M. R., \& O'Brien, A. D. (1998). Intiminmediated adherence of Shiga Toxin-producing Escherichia coli and attachingand-effacing pathogens. In Escherichia coli O157:H7 and other Shiga toxinproducing E. coli strains. J.B. Kaper and A.D. O'Brien, editors. American Society for Microbiology. Washington, DC, USA.

Kaper, J. B., Nataro, J. P., \& Mobley, H. L. T. (2004). Pathogenic Escherichia coli. Nat Rev Micro, 2(2), 123-140.

Karmali, M., Petric, M., Steele, B., \& Lim, C. (1983). Sporadic cases of haemolyticuraemic syndrome associated with faecal cytotoxin and cytotoxin-producing Escherichia coli in stools. The Lancet, 321(8325), 619-620.

Karmali, M. A., Petric, M., Lim, C., Cheung, R., \& Arbus, G. S. (1985). Sensitive method for detecting low numbers of verotoxin-producing Escherichia coli in mixed cultures by use of colony sweeps and polymyxin extraction of verotoxin. $J$. Clin. Microbiol., 22(4), 614-619.

Kenny, B. (1999). Phosphorylation of tyrosine 474 of the enteropathogenic Escherichia coli (EPEC) Tir receptor molecule is essential for actin nucleating activity and is preceded by additional host modifications. Molecular Microbiology, 31(4), 12291241.

Kenny, B., Abe, A., Stein, M., \& Finlay, B. B. (1997). Enteropathogenic Escherichia coli protein secretion is induced in response to conditions similar to those in the gastrointestinal tract. Infect. Immun., 65(7), 2606-2612.

Kenny, B., Ellis, S., Leard, A. D., Warawa, J., Mellor, H., \& Jepson, M. A. (2002). Coordinate regulation of distinct host cell signalling pathways by multifunctional enteropathogenic Escherichia coli effector molecules. Molecular Microbiology, 44(4), 1095-1107.

Khine, A. A., Tam, P., Nutikka, A., \& Lingwood, C. A. (2004). Brefeldin A and filipin distinguish two globotriaosyl ceramide\&sol;verotoxin-1 intracellular trafficking pathways involved in Vero cell cytotoxicity (Vol. 14): Oxford University Press. 
Kim, S. H., \& Kim, Y. H. (2004). Escherichia coli O157:H7 adherence to HEp-2 cells is implicated with curli expression and outer membrane integrity. Journal of Veterinary Science, 5(2), 119-124.

Kirkpatrick, C., Maurer, L. M., Oyelakin, N. E., Yoncheva, Y. N., Maurer, R., \& Slonczewski, J. L. (2001). Acetate and Formate Stress: Opposite Responses in the Proteome of Escherichia coli.J. Bacteriol., 183(21), 6466-6477.

Kwon, Y. M., \& Ricke, S. C. (1998). Induction of Acid Resistance of Salmonella typhimurium by Exposure to Short-Chain Fatty Acids. Appl. Environ. Microbiol., 64(9), 3458-3463.

Lasko, D. R., Zamboni, N., \& Sauer, U. (2000). Bacterial response to acetate challenge: a comparison of tolerance among species. Applied Microbiology and Biotechnology, 54(2), 243-247.

Law, D. (2000). Virulence factors of Escherichia coli 0157 and other Shiga toxinproducing E. coli. Journal of Applied Microbiology, 88(5), 729.

Lawhon, S. D., Maurer, R., Suyemoto, M., \& Altier, C. (2002). Intestinal short-chain fatty acids alter Salmonella typhimurium invasion gene expression and virulence through BarASirA. Molecular Microbiology, 46, 1451-1464.

Legrand-Defretin, V., Juste, C., Henry, R., \& Corring, T. (1991). Ion-pair highperformance liquid chromatography of bile salt conjugates: Application to pig bile. Lipids, 26(8), 578-583.

Lio, J., \& Syu, W., Jr. (2004). Identification of a negative regulator for the pathogenicity island of enterohemorrhagic Escherichia coli 0157:H7. Journal of Biomedical Science, 11(6), 855-863.

Louie, M., Read, S., Louie, L., Ziebell, K., Rahn, K., Borczyk, A., \& Lior, H. (2000). Molecular typing methods to investigate transmission of Escherichia coli O157:H7 from cattle to humans. Epidemiology and Infection, 123(01), 17-24.

Louise, C. B., Kaye, S. A., Boyd, B., Lingwood, C. A., \& Obrig, T. G. (1995). Shiga toxin-associated hemolytic uremic syndrome: effect of sodium butyrate on sensitivity of human umbilical vein endothelial cells to Shiga toxin. Infect. Immun., 63(7), 2766-2769.

Low, A. S., Dziva, F., Torres, A. G., Martinez, J. L., Rosser, T., Naylor, S., Spears, K., Holden, N., Mahajan, A., Findlay, J., Sales, J., Smith, D. G. E., Low, J. C., Stevens, M. P., \& Gally, D. L. (2006). Cloning, Expression, and Characterization of Fimbrial Operon F9 from Enterohemorrhagic Escherichia coli O157:H7. Infect. Immun., 74(4), 2233-2244. 
Lu, L., \& Walker, W. A. (2001). Pathologic and physiologic interactions of bacteria with the gastrointestinal epithelium. Am J Clin Nutr, 73(6), 1124S-1130.

Macfarlane, G. T., Gibson, G. R., \& Cummings, J. H. (1992). Comparison of fermentation reactions in different regions of the human colon. The Journal of applied bacteriology, 72(1), 57-64.

Malladi, V., Shankar, B., Williams, P. H., \& Balakrishnan, A. (2004). Enteropathogenic Escherichia coli outer membrane proteins induce changes in cadherin junctions of Caco-2 cells through activation of PKCa (Vol. 6): Elsevier Science.

Manjarrez-Hernandez, H. A., Baldwin, T. J., Williams, P. H., Haigh, R., Knutton, S., \& Aitken, A. (1996). Phosphorylation of myosin light chain at distinct sites and its association with the cytoskeleton during enteropathogenic Escherichia coli infection. Infect. Immun., 64(6), 2368-2370.

Mao, Y., Doyle, M. P., \& Chen, J. (2006). Role of colanic acid exopolysaccharide in the survival of enterohaemorrhagic Escherichia coli $\mathrm{O} 157: \mathrm{H} 7$ in simulated gastrointestinal fluids. Letters in Applied Microbiology, 42, 642-647.

March, S. B., \& Ratnam, S. (1986). Sorbitol-MacConkey medium for detection of Escherichia coli 0157 : $\mathrm{H} 7$ associated with hemorrhagic colitis. Journal of Clinical Microbiology, 23(5), 869.

Maroncle, N. M., Sivick, K. E., Brady, R., Stokes, F.-E., \& Mobley, H. L. T. (2006). Protease Activity, Secretion, Cell Entry, Cytotoxicity, and Cellular Targets of Secreted Autotransporter Toxin of Uropathogenic Escherichia coli. Infect. Immun., 74(11), 6124-6134.

Maurer, L. M., Yohannes, E., Bondurant, S. S., Radmacher, M., \& Slonczewski, J. L. (2005). pH Regulates Genes for Flagellar Motility, Catabolism, and Oxidative Stress in Escherichia coli K-12. J. Bacteriol., 187(1), 304-319.

McKee, M. L., Melton-Celsa, A. R., Moxley, R. A., Francis, D. H., \& O'Brien, A. D. (1995). Enterohemorrhagic Escherichia coli O157:H7 requires intimin to colonize the gnotobiotic pig intestine and to adhere to HEp-2 cells. Infect. Immun., 63(9), 3739-3744.

Mekalanos, J. J. (1992). Environmental signals controlling expression of virulence determinants in bacteria. J. Bacteriol., 174(1), 1-7.

Merrell, D. S., \& Camilli, A. (2002). Acid tolerance of gastrointestinal pathogens (Vol. 5): Elsevier Science.

Nataro, J. P., \& Kaper, J. B. (1998). Diarrheagenic Escherichia coli. Clin. Microbiol. Rev., 11(1), 142-201. 
Naylor, S. W., Low, J. C., Besser, T. E., Mahajan, A., Gunn, G. J., Pearce, M. C., McKendrick, I. J., Smith, D. G. E., \& Gally, D. L. (2003). Lymphoid FollicleDense Mucosa at the Terminal Rectum Is the Principal Site of Colonization of Enterohemorrhagic Escherichia coli O157:H7 in the Bovine Host. Infect. Immun., 7l(3), 1505-1512.

Nicholls, L., Grant, T. H., \& Robins-Browne, R. M. (2000). Identification of a novel genetic locus that is required for in vitro adhesion of a clinical isolate of enterohaemorrhagic Escherichia coli to epithelial cells. Molecular Microbiology, $35(2), 275-288$.

O'Connell, C. B., Creasey, E. A., Knutton, S., Elliott, S., Crowther, L. J., Luo, W., Albert, M. J., Kaper, J. B., Frankel, G., \& Donnenberg, M. S. (2004). SepL, a protein required for enteropathogenic Escherichia coli type III translocation, interacts with secretion component SepD. Molecular Microbiology, 52, 1613-1625.

O'Loughlin, E. V., \& Robins-Browne, R. M. (2001). Effect of Shiga toxin and Shiga-like toxins on eukaryotic cells Microbes and Infection, 3(6), 493-507.

Oh, M.-K., Rohlin, L., Kao, K. C., \& Liao, J. C. (2002). Global Expression Profiling of Acetate-grown Escherichia coli. J. Biol. Chem., 277(15), 13175-13183.

Osawa, R., Arakawa, E., Okitsu, T., Yamai, S., \& Watanabe, H. (2002). Levels of Thermostable Direct Hemolysin Produced by Vibrio parahaemolyticus O3:K6 and Other Serovars Grown Anaerobically with the Presence of a Bile Acid. Current Microbiology, 44(4), 302-305.

Ostroff, S. M., Griffin, P. M., Tauxe, R. V., Shipman, L. D., Greene, K. D., Wells, J. G., Lewis, J. H., Blake, P. A., \& Kobayashi, J. M. (1990). A Statewide Outbreak of Escherichia coli 0157: H7 Infections in Washington State. Am. J. Epidemiol., 132(2), 239-247.

Pace, J. L., Chai, T. J., Rossi, H. A., \& Jiang, X. (1997). Effect of bile on Vibrio parahaemolyticus. Appl. Environ. Microbiol., 63(6), 2372-2377.

Parkinson, J. S., \& Kofoid, E. C. (1992). Communication modules in bacterial signalling proteins. Annu. Rev. Genetics, 26, 71-112.

Paros, M., Tarr, P., Kim, H., Besser, T. E., \& Hancock, D. D. (1993). A comparison of human and bovine Escherichia coli $\mathrm{O} 157: \mathrm{H} 7$ isolates by toxin genotype, plasmid profile, and bacteriophage lambda-restriction fragment length polymorphism profile. The journal of infectious diseases, 168(5), 1300-1303.

Paton, A. W., Srimanote, P., Woodrow, M. C., \& Paton, J. C. (2001). Characterization of Saa, a Novel Autoagglutinating Adhesin Produced by Locus of Enterocyte 
Effacement-Negative Shiga-Toxigenic Escherichia coli Strains That Are Virulent for Humans. Infect. Immun., 69(11), 6999-7009.

Paton, J. C., \& Paton, A. W. (1998). Pathogenesis and Diagnosis of Shiga ToxinProducing Escherichia coli Infections. Clin. Microbiol. Rev., 11(3), 450-479.

Pazzi, P., Puviani, A. C., Dalla Libera, M. G., G., Ricci, D., Gullini, S., \& Ottolenghi, C. (1997). Bile salt-induced cytotoxicity and ursodeoxycholate cytoprotection: in vitro study in perfused rat hepatocytes. European Journal of Gastroenterology \& Hepatology, 9, 703-709.

Perna, N. T., Plunkett, G., Burland, V., Mau, B., Glasner, J. D., Rose, D. J., Mayhew, G. F., Evans, P. S., Gregor, J., Kirkpatrick, H. A., Pósfai, G., Hackett, J., Klink, S., Boutin, A., Shao, Y., Miller, L., Grotbeck, E. J., Davis, N. W., Lim, A., Dimalanta, E. T., Potamousis, K. D., Apodaca, J., Anantharaman, T. S., Lin, J., Yen, G., Schwartz, D. C., Welch, R. A., \& Blattner, F. R. (2001). Genome sequence of enterohaemorrhagic Escherichia coli O157:H7. Nature, 409(6819), 529-533.

Pizarro-Cerdá, J., \& Cossart, P. (2006). Bacterial Adhesion and Entry into Host Cells. Cell, 124(4), 715-727.

Polen, T., Rittmann, D., Wendisch, V. F., \& Sahm, H. (2003). DNA Microarray Analyses of the Long-Term Adaptive Response of Escherichia coli to Acetate and Propionate. Appl. Environ. Microbiol., 69(3), 1759-1774.

Pope, L. M., Reed, K. E., \& Payne, S. M. (1995). Increased protein secretion and adherence to HeLa cells by Shigella spp. following growth in the presence of bile salts. Infect. Immun., 63(9), 3642-3648.

Prouty, A. M., Brodsky, I. E., Falkow, S., \& Gunn, J. S. (2004). Bile-salt-mediated induction of antimicrobial and bile resistance in Salmonella typhimurium. Microbiology, 150(4), 775-783.

Prouty, A. M., \& Gunn, J. S. (2000). Salmonella enterica Serovar Typhimurium Invasion Is Repressed in the Presence of Bile. Infect. Immun., 68(12), 6763-6769.

Provenzano, D., Schuhmacher, D. A., Barker, J. L., \& Klose, K. E. (2000). The Virulence Regulatory Protein ToxR Mediates Enhanced Bile Resistance in Vibrio cholerae and Other Pathogenic Vibrio Species. Infect. Immun., 68(3), 1491-1497.

Puchowicz, M. A., Bederman, I. R., Comte, B., Yang, D., David, F., Stone, E., Jabbour, K., Wasserman, D. H., \& Brunengraber, H. (1999). Zonation of acetate labeling across the liver: implications for studies of lipogenesis by MIDA. Am J Physiol Endocrinol Metab, 277(6), E1022-1027. 
Pumbwe, L., Wareham, D. W., Aduse-Opoku, J., Brazier, J. S., \& Wexler, H. M. (2007). Genetic analysis of mechanisms of multidrug resistance in a clinical isolate of Bacteroides fragilis. Clinical Microbiology and Infection, 13(2), 183-189.

Ramos-Morales, F., Prieto, A. I., Beuzon, C. R., Holden, D. W., \& Casadesus, J. (2003). Role for Salmonella enterica Enterobacterial Common Antigen in Bile Resistance and Virulence. J. Bacteriol., 185(17), 5328-5332.

Ricke, S. C. (2003). Perspectives on the use of organic acids and short chain fatty acids as antimicrobials. Poult Sci, 82(4), 632-639.

Riordan, D. C., Duffy, G., Sheridan, J. J., Whiting, R. C., Blair, I. S., \& McDowell, D. A. (2000). Effects of Acid Adaptation, Product pH, and Heating on Survival of Escherichia coli O157:H7 in Pepperoni. Appl. Environ. Microbiol., 66(4), 17261729.

Rishi, P., Pathak, S., \& Ricke, S. C. (2005). Short Chain Fatty Acids Influence Virulence Properties of Salmonella Enterica Serovar Typhimurium. Journal of Environmental Science and Health, Part B, 40(4), 645 - 657.

Robinson, C. M., Sinclair, J. F., Smith, M. J., \& O'Brien, A. D. (2006). Shiga toxin of enterohemorrhagic Escherichia coli type 0157:H7 promotes intestinal colonization. Proceedings of the National Academy of Sciences, 103(25), 96679672.

Roe, A. J., McLaggan, D., Davidson, I., O'Byrne, C., \& Booth, I. R. (1998). Perturbation of Anion Balance during Inhibition of Growth of Escherichia coli by Weak Acids. J. Bacteriol., 180(4), 767-772.

Roe, A. J., Yull, H., Naylor, S. W., Woodward, M. J., Smith, D. G. E., \& Gally, D. L. (2003). Heterogeneous Surface Expression of EspA Translocon Filaments by Escherichia coli 0157:H7 Is Controlled at the Posttranscriptional Level. Infect. Immun., 71(10), 5900-5909.

Rosenshine, I., Ruschkowski, S., \& Finlay, B. B. (1996). Expression of attaching/effacing activity by enteropathogenic Escherichia coli depends on growth phase, temperature, and protein synthesis upon contact with epithelial cells. Infect. Immun., 64(3), 966-973.

Salmond, C. V., Kroll, R. G., \& Booth, I. R. (1984). The effect of food preservatives on $\mathrm{pH}$ homeostasis in Escherichia coli. Journal of general microbiology, 130(11), 2845-2850.

Sandvig, K., Garred, O., Prydz, K., Kozlov, J. V., Hansen, S. H., \& van Deurs, B. (1992). Retrograde transport of endocytosed Shiga toxin to the endoplasmic reticulum. Nature, 358(6386), 510-512. 
Sandvig, K., Ryd, M., Garred, O., Schweda, E., Holm, P. K., \& van Deurs, B. (1994). Retrograde transport from the Golgi complex to the ER of both Shiga toxin and the nontoxic Shiga B-fragment is regulated by butyric acid and cAMP. J. Cell Biol., 126(1), 53-64.

Sanger, J. M., Chang, R., Ashton, F., Kaper, J. B., \& Sanger, J. W. (1996). Novel form of actin-based motility transports bacteria on the surfaces of infected cells. Cell Motility and the Cytoskeleton, 34(4), 279-287.

Schmitt, C. K., Meysick, K. C., \& O'Brien, A. D. (1999). Bacterial Toxins: Friends or Foes? Emerging Infectious Diseases, 5(2), 224-234.

Schuhmacher, D. A., \& Klose, K. E. (1999). Environmental Signals Modulate ToxTDependent Virulence Factor Expression in Vibrio cholerae. J. Bacteriol., 181(5), $1508-1514$.

Segain, J. P., de la Bletiere, D. R., Bourreille, A., Leray, V., Gervois, N., Rosales, C., Ferrier, L., Bonnet, C., Blottiere, H. M., \& Galmiche, J. P. (2000). Butyrate inhibits inflammatory responses through NFkappa B inhibition: implications for Crohn's disease. Gut, 47(3), 397-403.

Sherman, P., Soni, R., Petric, M., \& Karmali, M. (1987). Surface properties of the Vero cytotoxin-producing Escherichia coli O157:H7. Infect. Immun., 55(8), 1824-1829.

Sinclair, J. F., \& O'Brien, A. D. (2002). Cell Surface-localized Nucleolin Is a Eukaryotic Receptor for the Adhesin Intimin-gamma of Enterohemorrhagic Escherichia coli O157:H7. J. Biol. Chem., 277(4), 2876-2885.

Spear, R. C., Seto, E., Remais, J., Carlton, E. J., Davis, G., Qiu, D., Zhou, X., Liang, Song, \& Fenwick, A. (2006). Fighting Waterborne Infectious Diseases. Science, 314(5802), 1081c-1083.

Sperandio, V., Li, C. C., \& Kaper, J. B. (2002). Quorum-Sensing Escherichia coli Regulator A: a Regulator of the LysR Family Involved in the Regulation of the Locus of Enterocyte Effacement Pathogenicity Island in Enterohemorrhagic $E$. coli. Infect. Immun., 70(6), 3085-3093.

Sperandio, V., Mellies, J. L., Nguyen, W., Shin, S., \& Kaper, J. B. (1999). Quorum sensing controls expression of the type III secretion gene transcription and protein secretion in enterohemorrhagic and enteropathogenic Escherichia coli. Proceedings of the National Academy of Sciences of the United States of America, 96(26), 15196-15201.

Sperandio, V., Torres, A. G., Giron, J. A., \& Kaper, J. B. (2001). Quorum Sensing Is a Global Regulatory Mechanism in Enterohemorrhagic Escherichia coli O157:H7. J. Bacteriol., 183(17), 5187-5197. 
Srimanote, P., Paton, A. W., \& Paton, J. C. (2002). Characterization of a Novel Type IV Pilus Locus Encoded on the Large Plasmid of Locus of Enterocyte EffacementNegative Shiga-Toxigenic Escherichia coli Strains That Are Virulent for Humans. Infect. Immun., 70(6), 3094-3100.

Stevens, M. P., Roe, A. J., Vlisidou, I., van Diemen, P. M., La Ragione, R. M., Best, A., Woodward, M. J., Gally, D. L., \& Wallis, T. S. (2004). Mutation of toxB and a Truncated Version of the efa-1 Gene in Escherichia coli O157:H7 Influences the Expression and Secretion of Locus of Enterocyte Effacement-Encoded Proteins but not Intestinal Colonization in Calves or Sheep. Infect. Immun., 72(9), 54025411 .

Stevens, M. P., van Diemen, P. M., Frankel, G., Phillips, A. D., \& Wallis, T. S. (2002). Efa1 Influences Colonization of the Bovine Intestine by Shiga Toxin-Producing Escherichia coli Serotypes O5 and O111. Infect. Immun., 70(9), 5158-5166.

Strockbine, N. A., Marques, L. R., Newland, J. W., Smith, H. W., Holmes, R. K., \& O'Brien, A. D. (1986). Two toxin-converting phages from Escherichia coli O157:H7 strain 933 encode antigenically distinct toxins with similar biologic activities. Infect. Immun., 53(1), 135-140.

Takahashi, M., Taguchi, H., Yamaguchi, H., Osaki, T., Komatsu, A., \& Kamiya, S. (2004). The effect of probiotic treatment with Clostridium butyricum on enterohemorrhagic Escherichia coli 0157:H7 infection in mice. FEMS Immunology and Medical Microbiology, 41(3), 219-226.

Tampakaki, A. P., Fadouloglou, V. E., Gazi, A. D., Panopoulos, N. J., \& Kokkinidis, M. (2004). Conserved features of type III secretion. Cellular Microbiology, 6(9), 805-816.

Tao, H., Bausch, C., Richmond, C., Blattner, F. R., \& Conway, T. (1999). Functional Genomics: Expression Analysis of Escherichia coli Growing on Minimal and Rich Media. J. Bacteriol., 181(20), 6425-6440.

Tarr, P. I., Bilge, S. S., Vary, J. C., Jr., Jelacic, S., Habeeb, R. L., Ward, T. R., Baylor, M. R., \& Besser, T. E. (2000). Iha: a Novel Escherichia coli O157:H7 AdherenceConferring Molecule Encoded on a Recently Acquired Chromosomal Island of Conserved Structure. Infect. Immun., 68(3), 1400-1407.

Tatsuno, I., Horie, M., Abe, H., Miki, T., Makino, K., Shinagawa, H., Taguchi, H., Kamiya, S., Hayashi, T., \& Sasakawa, C. (2001). toxB Gene on pO157 of Enterohemorrhagic Escherichia coli O157:H7 Is Required for Full Epithelial Cell Adherence Phenotype. Infect. Immun., 69(11), 6660-6669.

Torres, A. G., Giron, J. A., Perna, N. T., Burland, V., Blattner, F. R., Avelino-Flores, F., \& Kaper, J. B. (2002). Identification and Characterization of lpfABCC'DE, a 
Fimbrial Operon of Enterohemorrhagic Escherichia coli O157:H7. Infect. Immun., 70(10), 5416-5427.

Torres, A. G., Jeter, C., Langley, W., \& Matthysse, A. G. (2005a). Differential Binding of Escherichia coli O157:H7 to Alfalfa, Human Epithelial Cells, and Plastic Is Mediated by a Variety of Surface Structures. Appl. Environ. Microbiol., 71(12), 8008-8015.

Torres, A. G., Kanack, K. J., Tutt, C. B., Popov, V., \& Kaper, J. B. (2004). Characterization of the second long polar (LP) fimbriae of Escherichia coli O157:H7 and distribution of LP fimbriae in other pathogenic E. coli strains. FEMS Microbiology Letters, 238(2), 333-344.

Torres, A. G., \& Kaper, J. B. (2001). PAIs of intestinal Escherichia coli.

Torres, A. G., \& Kaper, J. B. (2003). Multiple Elements Controlling Adherence of Enterohemorrhagic Escherichia coli $\mathrm{O} 157: \mathrm{H} 7$ to HeLa Cells. Infect. Immun., 71(9), 4985-4995.

Torres, A. G., Tutt, C. B., Duval, L., Popov, V., Nasr, A. B., Michalski, J., \& Scaletsky, I. C. A. (2007). Bile salts induce expression of the afimbrial LDA adhesin of atypical enteropathogenic Escherichia coli. Cellular Microbiology, 9(4), 10391049.

Torres, A. G., Zhou, X., \& Kaper, J. B. (2005b). Adherence of Diarrheagenic Escherichia coli Strains to Epithelial Cells. Infect. Immun., 73(1), 18-29.

Touzé, T., Hayward, R. D., Eswaran, J., Leong, J. M., \& Koronakis, V. (2004). Selfassociation of EPEC intimin mediated by the $\beta$-barrel-containing anchor domain: a role in clustering of the Tir receptor. Molecular Microbiology, 51(1), 73-87.

Tzipori, S., Karch, H., Wachsmuth, K. I., Robins-Browne, R. M., O'Brien, A. D., Lior, H., Cohen, M. L., Smithers, J., \& Levine, M. M. (1987). Role of a 60-megadalton plasmid and Shiga-like toxins in the pathogenesis of infection caused by enterohemorrhagic Escherichia coli $\mathrm{O} 157: \mathrm{H} 7$ in gnotobiotic piglets. Infect. Immun., 55(12), 3117-3125.

Uhlich, G. A., Keen, J. E., \& Elder, R. O. (2001). Mutations in the csgD Promoter Associated with Variations in Curli Expression in Certain Strains of Escherichia coli O157:H7. Appl. Environ. Microbiol., 67(5), 2367-2370.

Vanmaele, R. P., \& Armstrong, G. D. (1997). Effect of carbon source on localized adherence of enteropathogenic Escherichia coli. Infect. Immun., 65(4), 14081413. 
Wang, Y. (2002). The Function of OmpA in Escherichia coli (Vol. 292): Elsevier Science.

Wells, T. J., Sherlock, O., Rivas, L., Mahajan, A., Beatson, S. A., Torpdahl, M., Webb, R. I., Allsopp, L. P., Gobius, K. S., Gally, D. L., \& Schembri, M. A. (2008). EhaA is a novel autotransporter protein of enterohemorrhagic Escherichia coli O157:H7 that contributes to adhesion and biofilm formation. Environmental Microbiology, 10, 589-604.

Wen, S. X., Teel, L. D., Judge, N. A., \& O'Brien, A. D. (2006). Genetic toxoids of Shiga toxin types 1 and 2 protect mice against homologous but not heterologous toxin challenge (Vol. 24): Elsevier Science.

Wilson, R. K., Shaw, R. K., Daniell, S., Knutton, S., \& Frankel, G. (2001). Role of EscF, a putative needle complex protein, in the type III protein translocation system of enteropathogenic Escherichia coli. Cellular Microbiology, 3, 753-762.

Xicohtencatl-Cortes, J., Monteiro-Neto, V., Ledesma, M. A., Jordan, D. M., Francetic, O., Kaper, J. B., Puente, J. L., \& Girón, J. A. (2007). Intestinal adherence associated with type IV pili of enterohemorrhagic Escherichia coli $\mathrm{O} 157: \mathrm{H} 7$. The Journal of Clinical Investigation, 117(11), 3519-3529.

Yoh, M., Bi, Z., Matsuyama, J., Nagayama, K., \& Honda, T. (2003). Effect of Environmental Conditions on Proteins Secreted by Enterohemorrhagic Escherichia coli O26: H11. Microbiol Immunol, 47(1), 1-6.

Zhang, L., Chaudhuri, R. R., Constantinidou, C., Hobman, J. L., Patel, M. D., Jones, A. C., Sarti, D., Roe, A. J., Vlisidou, I., Shaw, R. K., Falciani, F., Stevens, M. P., Gally, D. L., Knutton, S., Frankel, G., Penn, C. W., \& Pallen, M. J. (2004). Regulators Encoded in the Escherichia coli Type III Secretion System 2 Gene Cluster Influence Expression of Genes within the Locus for Enterocyte Effacement in Enterohemorrhagic E. coli O157:H7. Infect. Immun., 72(12), 72827293. 